Aus der Klinik für Dermatologie, Venerologie und Allergologie

(Prof. Dr. med. M. P. Schön)

der Medizinischen Fakultät der Universität Göttingen

\title{
Epikutantests mit parallel zur Standardreihe untersuchten Substanzen an Patienten der \\ Dermatologischen Universitätsklinik Göttingen
}

\author{
INAUGURAL- DISSERTATION \\ zur Erlangung des Doktorgrades \\ der Medizinischen Fakultät der \\ Georg- August- Universität zu Göttingen
}

vorgelegt von

Mohammad Reza Safaei Nikoie

aus

Teheran/Iran

Göttingen 2018 
Dekan:

Referent/in

Ko-Referent/in:

Drittreferent/in:
Prof. Dr. rer. nat. H. K. Kroemer

Prof. Dr. T. Fuchs

Datum der mündlichen Prüfung: 
Hiermit erkläre ich, die Dissertation mit dem Titel "Epikutantests mit parallel zur Standardreihe untersuchten Substanzen an Patienten der Dermatologischen Universitätsklinik Göttingen" eigenständig angefertigt und keine anderen als die von mir angegebenen Quellen und Hilfsmittel verwendet zu haben.

Göttingen, den 
1. Allgemeiner Teil 1

1.1 Einleitung $\quad 1$

1.2 Geschichte des Epikutantests 3

1.3 Pathophysiologische Grundlagen des allergischen Kontaktekzems $\quad 4$

1.4 Der Epikutantest 5

1.4.1 Indikationen $\quad 6$

$\begin{array}{ll}\text { 1.4.2 Kontraindikationen } & 6\end{array}$

1.4.3 Applikation und Vehikel $\quad 8$

1.4.4 Ablesung und Interpretation der Reaktion 9

$\begin{array}{ll}1.5 \text { MOAHLFA-Index } & 10\end{array}$

1.6 Allergene der Standardreihe und andere Testreihen 11

1.7 Anforderungen zur Aufnahme von Allergenen in die Standardreihe $\quad 12$

$\begin{array}{ll}1.8 \text { Hitliste } & 14\end{array}$

1.9 Fragestellung 14

2. Material und Methoden $\quad 15$

3. Ergebnisse 16

3.1 Analyse der Patientendaten 16

$\begin{array}{ll}3.2 \text { Testsubstanzen und Ergebnisse der Epikutantests } & 17\end{array}$

3.3 Ergebnisse der Epikutantests (1996-2004) 21

3.4 Analyse des MOAHLFA-Index 24

4. Diskussion 26

4.1 Testpopulation 26

4.2 Interpretation der Ergebnisse der Substanzen der Ergänzungsreihen 27

- Amalgam 27

- p-Aminomethylbenzolsulfonamid (Mafenid) 28

- Bioban CS $1246 \quad 28$

- Bronidox L (5-Brom-5-Nitro-1,3-dioxan) 29

- Bufexamac 30

- Cain-Mix 1

- Carba-Mix 32

- Carboxymethylcellulose 33

- Clobetasol-17-propionat 34

- Dexpanthenol 35

- Dibromdicyanobutan 36 
- Dimethylformamid

- 1,3-Diphenylguanidin 38

- Dispersions Blau 124 und Mix 124/106 39

- Glutaraldehyd 40

- Hexylzimtaldehyd 41

- Hg.-Präcipitatsalbe $\quad 42$

- Hydrocortison-17-butyrat 43

- Imidazolidinylharnstoff (Germall 115) 44

- Incidin Plus 45

- Iodpropinylbutylcarbamat $\quad 45$

- KKM 702

- Kolophonium-Mix II $\quad 47$

- Latexhandschuhextrakt (4\%ige Pricklösung) 48

- Lyral (4-(4-Hydroxy-4-methylpentyl)-3-cyclohexen-1-carboxaldehyd) 49

- 2-Mercaptobenzimidazol 50

- Mercaptobenzothiazol 50

- Myristylalkohol

- Natriumdisulfit

- Natriumlaurylsulfat $\quad 54$

- Phenoxyethanol 54

- Poly(hexamethylendiguanid)-HCL 55

- Prednicarbat

- Propolis

- Propylenglykol 58

- Quaternium-15

$\begin{array}{ll}\text { - Sesquiterpenlactone-Mix } & 60\end{array}$

- Tantalum und Tantalum-V-Chlorid 61

- Teebaumöl $\quad 62$

$\begin{array}{lc}\text { - Tixocortol-pivalat } & 63\end{array}$

- Toluolsulfonamid-Formaldehydharz 65

- p-Toluyldiamin 66

- Unguentum leniens

$\begin{array}{ll}\text { - Zinkdibenzyldithiocarbamat } & 67\end{array}$

$\begin{array}{ll}\text { - Zinkdibutyldithiocarbamat } & 68\end{array}$

$\begin{array}{ll}\text { 5. Zusammenfassung } & 69\end{array}$

6. Anhang 71

$\begin{array}{ll}\text { 7. Literaturverzeichnis } & 105\end{array}$ 


\section{Verzeichnis der Abkürzungen}

$\begin{array}{ll}\text { AKE } & \text { Allergisches Kontaktekzem } \\ \text { DDG } & \text { Deutsche Dermatologische Gesellschaft } \\ \text { DEP. } & \text { Diethylphthalat } \\ \text { DKG } & \text { Deutsche Kontaktallergie Gruppe } \\ \text { Eth. } & \text { Ethanol } \\ \text { ICDRG } & \text { International Contact Research Group } \\ \text { IVDK } & \text { Informationsverbund Dermatologischer Kliniken } \\ \text { irr. } & \text { irritativ } \\ \text { NACDG } & \text { North American Contact Dermatitis Group } \\ \text { neg. } & \text { negativ } \\ \text { Oli. } & \text { Olivenöl } \\ \text { Petr. } & \text { Petroleum } \\ \text { pos. } & \text { Positiv } \\ \text { syn. } & \text { Synonym } \\ \text { Vas. } & \text { Vaseline }\end{array}$




\section{Allgemeiner Teil}

\subsection{Einleitung}

Die Immunabwehr ist eine Schutzeinrichtung des Organismus vor internen und externen Noxen. Gelegentlich führt jedoch diese Schutzeinrichtung zu einer überschießenden Reaktion, die eine Schädigung des Organismus zur Folge haben kann. Bei dieser überschießenden Reaktion spricht man von einer Allergie (Bandmann 1977). Tritt eine solche Allergie nach Kontakt von Noxen auf der Haut auf, spricht man von einer Kontaktdermatitis, wobei der Begriff Kontaktekzem synonym verwendet wird. Der Begriff Dermatitis beschreibt eher allgemein gefasst entzündliche Hautveränderungen durch Stoffe, die mehr oder weniger bei jedem hervorgerufen werden können. Ein Ekzem (Dermatitis ekzematosa) bezieht sich eher auf Hautveränderungen, die eine Reaktion auf Sensibilisierungen auf bekannte oder unbekannte Stoffe sind. Somit kann ein Ekzem durch einen primär nicht pathologischen Reiz hervorgerufen werden (Bandmann 1977).

Nachfolgend wird vereinfachend von Kontaktekzem gesprochen. Mit einer Inzidenz von 3 pro 1000 ist das Kontaktekzem die häufigste Erkrankung der Haut (Schnuch et al. 2002b) und sie ist mit einer Prävalenz von 5-7\% vergleichbar mit anderen wichtigen Volkskrankheiten wie z.B. Diabetes mellitus, welche eine Prävalenz von etwa 5\% hat (Schnuch et al. 2002b).

Es lassen sich pathogenetisch zwei Hauptformen des Kontaktekzems unterscheiden: das allergische und das toxisch-irritative Kontaktekzem. Das allergische Kontaktekzem (AKE) stellt eine zellvermittelte Allergie, sogenannte Spättyp- oder Typ-IV-Allergie nach Coombs und Gell dar (Enk und Knop 2007). Voraussetzung für das AKE ist eine immunologische Reaktion nach einem vorherigen Kontakt mit einer Noxe (Kontaktallergen) und eine überschießende Reaktion bei erneutem Kontakt (Enk und Knop 2007). Das toxisch-irritative Kontaktekzem ist eine primär nicht immunologische Entzündungsreaktion auf physikalische oder chemische Stoffe, sog. Irritantien (Büchner 2001).

Die Klinik eines akuten AKE ist durch eine entzündliche Reaktion gekennzeichnet, die konsekutiv zu einer Reduzierung der allergentragenden Zellen führen kann (Büchner 2001). Dabei kann es zu einer Sensibilisierung kommen. Etwa 48-72 Stunden nach Exposition zeigt sich am Ort des Allergenkontaktes ein Ödem, erythematöse Papeln sowie Bläschen, die zerplatzen und nässende Oberflächen bilden können. Im Gegensatz zum irritativen Geschehen kann es beim AKE zu Streureaktionen an kontaktfernen Körperstellen kommen (Enk und Knop 2007). Bei fortgesetztem Allergenkontakt zeigen sich im chronischen Stadium häufig Rötungen, Bläschen, Krusten, Erosionen und Schuppen nebeneinander. Typisch ist zudem 
eine Lichenifikation, die durch eine entzündliche Verdickung und Vergröberung der Hautfelderung gekennzeichnet ist (Büchner 2001).

Diagnose und Ursachenklärung eines Ekzems stellen eine Herausforderung dar, da sich trotz Unterschieden in Ätiologie, Pathogenese und Klinik die verschiedenen Typen sich in ihrer Reaktionscharakeristik ähneln können. Die korrekte Diagnose der unterschiedlichen Ekzemformen ist wichtig für die Therapie, da die Konsequenzen für den Patienten sowohl im Alltag als auch im Berufsleben erheblich sind. In vielen Ländern rangiert die AKE mit einem Anteil von 30\% auf Platz eins aller Berufskrankheiten und in 90-95\% der Berufsdermatosen sind ursächlich Kontaktallergien zu finden (Diepgen und Kanerva 2006). In den USA verursacht die AKE mit einem Anteil von $7 \%$ an allen Berufskrankheiten ein jährliches Kostenvolumen von über 250 Millionen US-Dollar (Belsito 2000). Für den Patienten individuell kann ein Kontaktekzem zur Folge haben, dass er seinen erlernten Beruf nicht mehr ausüben kann.

Die Sensibilisierung auf Noxen oder Allergene ist eine bleibende Veränderung des Immunsystems und nicht heilbar (Schnuch et al. 2005c). Untersuchungen in Niedersachsen Anfang der 1990er Jahre zeigten beispielhaft, dass fast jeder dritte Friseurlehrling bereits während der Ausbildung ein Ekzem entwickelte und für jeden fünften ein Berufswechsel unausweichlich wurde (Budde und Schwanitz 1991).

Eine differenzierte Diagnostik und Prävention vom AKE, dessen Prävalenz in den letzten Jahren zugenommen hat, ist zunehmend wichtiger. Durch die Anamnese und körperliche Untersuchung allein lässt sich jedoch die Diagnose des Kontaktekzems nicht immer bestimmen. Die entscheidende diagnostische Hilfe ist der im 19. Jahrhundert entwickelte Epikutantest (Foussereau 1984). Dabei handelt es sich um eine - gemessen am Aufwand höchst effektive Untersuchung, um ein akutes AKE in einem umschriebenen Bereich hervorzurufen.

Angesichts von mehr als 2800 potentiellen Allergenen in der Umwelt (Belsito 2000) ist man international bemüht, eine Standardisierung der Testsubstanzen, Applikationsdauer, Testvehikel, Ableserichtlinien und Ablesezeitpunkte zu erreichen, um die Durchführung des Epikutantests zu optimieren und die Ergebnisse vergleichbar zu machen. Internationale (wie die „International Contact Dermatitis Research Group“ ICDRG oder die „North American Contact Dermatitis Group“ NACDG) und nationale Gremien (wie die „Deutsche Kontaktallergie Gruppe“ DKG, oder der „Informationsverbund Dermatologischer Kliniken“ IVDK) sprechen auf der Grundlage von wissenschaftlichen Untersuchungen Empfehlungen aus, wie solche Standardisierungen aussehen sollten. Darüber hinaus kann es auch sinnvoll 
sein, spezielle Fragestellungen abhängig von lokalen Gegebenheiten zu untersuchen (Wahlberg et al. 2006).

Es gibt weitere diagnostische Möglichkeiten, wie zum Beispiel den Lymphozytentransformationstest oder auch den MELISA-In-Vitro Test (Brehler und Merk 2005). Jedoch sind diese Untersuchungen im Vergleich zum Epikutantest aufwändiger und im Ergebnis weniger eindeutig. Somit bleibt der Epikutantest bislang das einzige für die Routinediagnostik geeignete Instrument zum Nachweis einer epidermalen Sensibilisierung und AKE.

\subsection{Geschichte des Epikutantests}

Die Universität Göttingen und ihre Forscher spielen in der Historie des Epikutantests und der Kontaktdermatitis bis heute eine entscheidende Rolle, sowohl national als auch international (Scholz 2007).

Bereits 1847 beschrieb der Göttinger Chemiker Städeler detailliert eine Art Epikutantest, um allergische Reaktionen hervorzurufen. Dabei wurde ein „Stückchen Löschpapier“, welches vorher in Balsam getränkt wurde, auf ein $1 \mathrm{~cm}^{2}$ großes Hautareal aufgetragen. Nach 3 Stunden wurden unter dem Papier brennende kleine Bläschen beschrieben (Foussereau 1984). Als Erfinder des Epikutantests wird hingegen der Breslauer Dermatologe Josef Jadassohn angesehen, der einen Teil seines Medizinstudiums an der Universität Göttingen absolvierte. Er stellte 1895 auf einem Kongress in Graz seine „Applikationsmethode“ vor, bei der Iodoform und Quecksilberderivate eine Rolle spielten (Foussereau 1984). Er versuchte bei bereits sensibilisierten Patienten das klinische Bild einer Kontaktdermatitis hervorzurufen. Man kann dabei davon ausgehen, dass seine Überlegungen von den Beobachtungen und Erkenntnissen seines Abteilungsleiters in Breslau, Albert Neisser, geprägt worden waren. Dieser hatte 1884 ebenfalls Dermatitiden durch quecksilberhaltige Substanzen und insbesondere Iodoform ausgelöst (Foussereau 1984). In den folgenden Jahren wurden verschiedene Bezeichnungen für die Epikutantests verwendet, zum Beispiel funktionelle Hautprüfung (1904 Jadassohn). Schließlich führte 1916 Cooke den bis heute gebräuchlichen englischen Namen „Patch Test“ ein (Foussereau 1984). In der Folgezeit waren viele Dermatologen an der Weiterentwicklung der Diagnostik der Kontaktdermatitis beteiligt. 1938 wurde von Poul Bonnevie in Kopenhagen die erste Liste von Allergenen veröffentlicht, die routinemäßig getestet werden sollten. Noch heute sind 1/3 der dort erwähnten 21 Allergene in der aktuellen Standardreihe vertreten (Wahlberg et al. 2006). 
Von der Arbeitsgemeinschaft der Deutschen Dermatologischen Gesellschaft (DDG) wurde 1987 die Deutsche Kontaktallergie Gruppe (DKG) als die Instanz, die Empfehlungen im deutschsprachigen Raum bezüglich Epikutantests aussprechen soll, gegründet (Schnuch et al. 2012).

1988 wurde der Informationsverbund Dermatologischer Kliniken (IVDK) gegründet. Der IVDK war anfangs eine Gruppe mit 8 Mitgliedern mit Sitz in Göttingen und deren Aufgabe ist u.a., systematisch epidemiologische Daten von Epikutantests zu erheben (Schnuch et al. 2012). Diesem Verbund gehören nun mittlerweile 56 Kliniken aus Deutschland, Österreich und der Schweiz an, und sie werden durch den DKG im Council der European Society of Contact Dermatitis repräsentiert (Schnuch et al. 2012).

\subsection{Pathophysiologische Grundlagen des allergischen Kontaktekzems}

Das allergische Kontaktekzem (ICD-10: L23.9) ist eine zellvermittelte Reaktion vom Spättyp (Typ-IV-Reaktion nach Coombs und Gell), die eine spezifische Sensibilisierung auf einen Umweltstoff (Kontaktallergen) voraussetzt. Das Resultat ist eine zeitlich versetzte Reaktion auf der Haut, die nach der Definition von Galen alle Zeichen einer Entzündung beinhalten kann: Überwärmung (Calor), Erythem (Rubor), Pruritus als Vorstufe von Schmerz (Dolor), Exsudat bzw. Infiltrate (Tumor) und die Functio laesa im Sinne einer reduzierten Schutzfunktion gegenüber allen, auch schwachen Reizen (Enk und Knop 2007).

Häufig sind Kontaktallergene niedermolekulare Substanzen, sogenannte Haptene. Ihr Molekulargewicht ist zu klein, um selbstständig als Allergen fungieren zu können. In der Epidermis werden sie durch Bindung an körpereigene Moleküle, den sogenannten HaptenCarrier-Komplex, zu Allergenen. Dabei werden die Allergene von immunkompetenten Langerhans-Zellen (dendritischen Zellen) aufgenommen und an MHC-Klasse-II gebunden. Die nun aktivierten Langerhans-Zellen wandern über Lymphbahnen zu lokalen Lymphknoten und präsentieren diese Allergene zirkulierenden CD4+-T-Lymphozyten, die sich wiederum in sogenannte Memory-T-Zellen, auch Effektor-Zellen genannt, umwandeln (Büchner 2001). Die Memory-T-Zellen sind nun sensibilisiert und verlassen den Lymphknoten und breiten sich im gesamten Körper aus. Diese Phase kann bis zu 14 Tage dauern.

Bei wiederholtem Kontakt mit dem Allergen und erneuter Präsentation durch LangerhansZellen erkennen die Memory-T-Zellen die Allergene wieder und führen über die Freisetzung von Zytokinen und Mediatoren, wie zum Beispiel IL-1, IL-6, GM-CSF, TNFa, zum Einstrom von verschiedenen Zellpopulationen, die eine inflammatorische Reaktion hervorrufen (Büchner 2001). Innerhalb von Stunden sind erste histologische Veränderungen zu sehen. 
Monozyten infiltrieren die Epidermis und häufen sich um Blutgefässe und Schweißdrüsen an. Ein Maximum wird innerhalb von 48-72 Stunden erreicht, wobei Makrophagen, basophile Granulozyten und Mastzellen zum Vollbild des Infiltrates beitragen, häufig begleitet von parazellulärem Ödem (Büchner 2001). Im weiteren Verlauf wird die Reaktion langsam durch immunsuppressorische Mediatoren wie Prostaglandin E aus Makrophagen abgeschwächt. In aktivierten Keratinozyten, die in der Haut verteilt aufzufinden sind, wird enzymatisch der Hapten-Carrier-Komplex abgebaut, und schließlich kann sich die Entzündung, wenn auch selten, zurückbilden. Residuen können auf der Haut sichtbar als Narben oder Lichenifikationen zurückbleiben (Büchner 2001).

Auf ähnliche Weise kann grundsätzlich anstatt einer Allergie auch eine Toleranz gegenüber Umweltstoffen entwickelt werden, wobei der exakte Mechanismus nicht genau bekannt ist (Becker 2013). Hierbei spielen unter anderen Suppressor-T-Zellen, Antikörper gegen T-ZellRezeptoren, aber auch die Konfiguration des Hapten-Carrier-Komplex eine Rolle. Untersuchungen konnten zeigen, dass potente Kontaktallergene durch Bindung an andere Carrier zu einer Toleranzentwicklung anstatt zu einer allergischen Reaktion beitragen können (Belsito 2000). Die Dichte der Langerhans-Zellen in der Haut scheint ebenfalls eine Rolle bei der Manifestation der AKD zu spielen. Untersuchungen zeigten, dass allergische Reaktionen auf der dünneren dorsalen Seite der Haut ausgeprägter sind als auf der dickeren palmaren Seite, wo Langerhans Zellen eine geringere Dichte haben (Belsito 2000).

\subsection{Der Epikutantest}

Bei einem Epikutantest (synonym: patch test) wird durch einen allergologisch geschulten Arzt künstlich eine allergische Kontaktdermatitis in einem umschriebenen Bereich mittels Testpflaster provoziert. Vor jedem Epikutantest müssen bei der Anamnese Informationen bezüglich Atopie, Beruf und Allergenexposition eingeholt werden. Weiterhin muss der Hautzustand auf seine Testfähigkeit überprüft werden. Entsprechend den Leitlinien der Deutschen Dermatologischen Gesellschaft (DDG) und Empfehlungen der Deutschen Kontaktdermatitis Gruppe (DKG), die im Jahre 1998 definiert und zuletzt im Jahre 2007 (Schnuch et al. 2008) überarbeitet wurden, werden nach einheitlichen Kriterien die Epikutantests durchgeführt (Schnuch et al. 2001). Die synchrone Reproduzierbarkeit wird in der Literatur in Abhängigkeit von der Testmethode und dem Allergen mit $60 \%$ bis $90 \%$ angegeben (Brasch et al. 1994).

Die Applikationsdauer beträgt im Allgemeinen 48 Stunden, und der klinisch relevante Ablesezeitpunkt ist 72 Stunden nach Entfernen des Testpflasters. 


\subsubsection{Indikationen}

Entsprechend den oben genannten Leitlinien gelten folgende Indikationen für die Durchführung von Epikutantests (s. Abbildung 1) (Schnuch et al. 2008):

1. Bei klinischem Verdacht auf eine kontaktallergische Reaktion der Haut oder hautnahen Schleimhaut

2. Bei Verdacht auf eine allergisch bedingte Berufsdermatose

3. Bei ätiologisch ungeklärter Dermatitis

4. Bei Verdacht einer Provozierung oder Verschlimmerung einer bestehenden Dermatose, zum Beispiel Neurodermatitis oder Psoriasis

5. Zur Diagnostik von medikamentös bedingten Exanthemen

\subsubsection{Kontraindikationen}

Einschränkungen, bei denen ein Epikutantest nicht durchgeführt werden sollte, sind nach Schnuch et al. 2008:

1. Vorliegen von Faktoren, die das Ergebnis verfälschen können: intensive UVExposition, längere Vorbehandlung mit topischen Glukokortikosteroiden, Einnahme von immunsuppressiven oder immunmodulierenden Medikamente

2. bekannte Schwangerschaft, wegen eventueller Beeinflussung des Ergebnisses durch hormonelle Umstellung und mögliche teratogene Wirkung

Unerwünschte Wirkungen durch den Test, wie eine mögliche iatrogene Sensibilisierung, müssen mit dem Patienten im Vorfeld in einem Aufklärungsgespräch besprochen werden. Bei fraglichen Ergebnissen ist ein erneuter Test frühestens nach 2 Monaten zu empfehlen. Bei sicherem Nachweis einer Sensibilisierung auf ein Allergen sollte eine Testwiederholung vermieden werden. 


\section{Epikutantest}

Anamnese und Klinik:

Verdacht auf Kontaktallergie (weitere Indikationen: siehe Abs. 2)
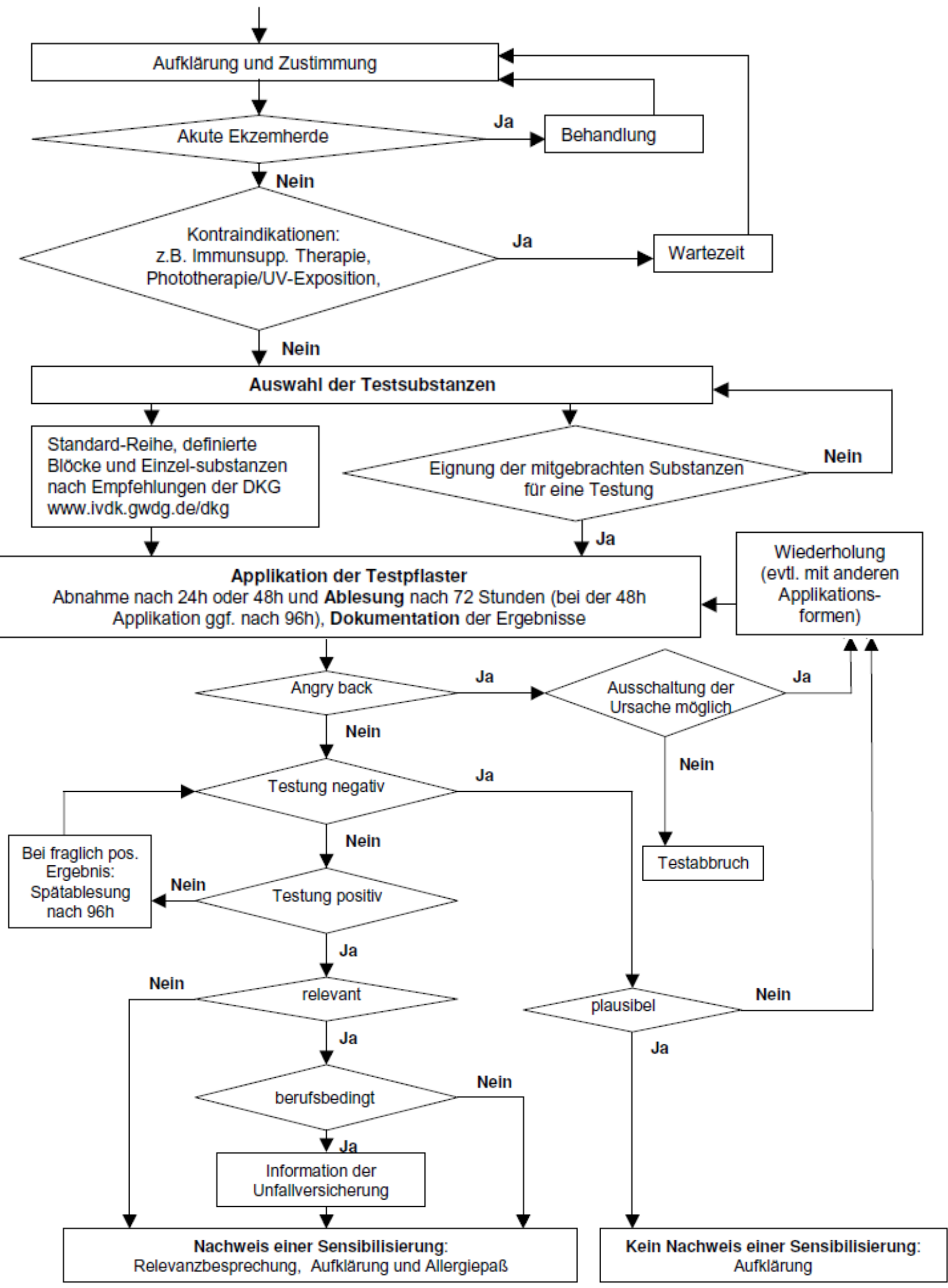

Abb. 1: Algorithmus des Epikutantests (Schnuch et al. 2008) 


\subsubsection{Applikation und Vehikel}

Der Epikutantest ist ein geschlossener Test. Die Testsubstanz wird in eine Trägersubstanz, das Vehikel, eingearbeitet und mit einem Testpflaster vertikal auf die Haut des oberen Rückens mit 2-4 cm Abstand zur Mittellinie befestigt. Die Ränder werden vor dem Entfernen z. B. einem wasserfesten Stift markiert. Bei dem Testpflaster handelt es sich üblicherweise um das Finn-Chamber ® System, welches 1975 von Pirilä eingeführt wurde. Es handelt sich um ein hautverträgliches Scanpor-Vlies-Verbandspflaster von $50 \mathrm{~mm}$ Breite. Darauf sind in Doppelreihen 5 oder 10 runde Aluminiumkammern von etwa $8 \mathrm{~mm}$ Durchmesser befestigt. Durch die eingesetzte impermeable Abdeckung kann das Hautareal begrenzt, die Verteilung gleichmäßig ermöglicht und die Testsubstanz relativ dicht aufgebracht werden (Fuchs 2007a). Durch den Einsatz eines luftdurchlässigen und hautverträglichen Verbandspflasters wird eine Pflasterreaktion und somit ein Erschweren der Ergebnisbeurteilung verhindert.

Das meist verwendete Testvehikel ist Vaseline (Vas.), da diese die Eigenschaften hat, einen guten Kontakt zwischen Haut und Testsubstanz zu gewährleisten und zudem kostengünstig ist (Wahlberg 1995). Weitere verwendete Vehikel sind Wasser (Aqua), Olivenöl (Oli.), und Diethylphthalat (DEP.). Diese haben prinzipiell gegenüber der Vaseline den Nachteil, dass sie sich verflüchtigen können und somit die Konzentration der zugeführten Substratmenge variieren kann (Wahlberg 1995). Gemäß den Leitlinien der DKG beträgt die empfohlene Expositionszeit 48 Stunden, jedoch sind grundsätzlich auch 24 Stunden möglich. Bislang gibt es keinen ausreichenden Beleg für eine Überlegenheit einer der beider Expositionszeiten (Schnuch et al. 2001). 


\subsubsection{Ablesung und Interpretation der Reaktion}

Gemäß der Empfehlung der DKG, erfolgt die Ablesung der Reaktion 72 Stunden nach Entfernen des Testpflasters, bei einer Expositionszeit von 48 Stunden (Schnuch et al. 2001). Zur Beurteilung der Testreaktion, bei der die Morphologie die entscheidende Rolle spielt, ist dies der optimale Zeitpunk (Fuchs 2007a).

Reaktionen im Epikutantest werden folgendermaßen abgelesen und codiert (Schnuch, et al. 2008):

\begin{tabular}{|c|l|l|}
\hline Symbol & \multicolumn{1}{|c|}{ Morphe } & \multicolumn{1}{c|}{ Bedeutung } \\
\hline- & keine Reaktion & negativ \\
\hline$?$ & nur Erythem, kein Infiltrat & allergisch, irritativ oder unklar \\
\hline$f$ & wenige follikuläre Papeln & allergisch, irritativ oder unklar \\
\hline+ & Erythem, Infiltrat, diskrete Papeln & einfach positive allergische Reaktion \\
\hline++ & Erythem, Infiltrat, Papeln, Vesikel & zweifach positive allergische Reaktion \\
\hline+++ & Erythem, Infiltrat, konfluierende Vesikel & dreifach positive allergische Reaktion \\
\hline ir & Seifeneffekt, Ringeffekt, Blase, Nekrose & irritative Reaktion \\
\hline
\end{tabular}

Zur Beurteilung der Reaktionsdynamik sollten 30 Minuten und 24 Stunden nach Entfernen des Pflasters Ablesungen erfolgen. Die Reaktionsdynamik kann bei der Unterscheidung zwischen einer allergischen und irritativen Reaktion wichtige Informationen über die Genese geben. Weitere Kriterien zur Unterscheidung sind nach Fuchs 2007a:

\begin{tabular}{|l|l|}
\hline Allergische Reaktion & Irritative Reaktion \\
\hline unscharfe Begrenzung & relativ scharfe Begrenzung \\
intensives Erythem & Erythem, nicht selten mit bräunlichem Farbton \\
Papeln, Vesikel, Infiltration & Blasen, evtl. Nekrosen und Pustelbildung \\
relativ langsame Entwicklung (Crescendo) & schnelle Entwicklung und Abklingen (Decrescendo) \\
längere Persistenz & relativ kurze Persistenz „,effect du savon“ \\
\hline
\end{tabular}

Um die Irritabilität der Haut zum Zeitpunkt des Epikutantests zu erfassen, kann gleichzeitig ein Irritans, wie z.B. Natriumlaurylsulfat, appliziert werden. Eine unspezifische Rötung nach 24-48 Stunden Exposition deutet auf eine erhöhte Hautempfindlichkeit hin und hilft bei der Interpretation von schwach bzw. fraglich positiven Reaktionen, $\mathrm{d}$. $\mathrm{h}$. besonders von Erythemen (Schnuch et al. 2001). Einfach positive Reaktionen sollten besonders bei unklarer Anamnese eher zurïckhaltend und nicht als allergisch gedeutet werden. 
Ein weiterer hilfreicher Parameter zur Interpretation von allergischen Reaktionen ist der sogenannte Reaktion Index (RI). Es ist definiert als: $(a-q-i) /(a+q+i)$, wobei a = allergische Reaktionen, $\mathrm{q}$ = fragliche Reaktionen und $\mathrm{i}$ = irritative Reaktionen sind (Geier et al. 2003d). Dieser Index hat eine Spanne von -1, wenn alle beobachteten Testreaktionen fraglich oder irritativ sind, bis +1 , wenn sich nur positive Testreaktionen zeigen (Geier et al. 2003d). Der RI hilft vor allem bei schwach positiven Reaktionen eine Differenzierung vorzunehmen (Brasch et al. 1995).

Bei der Bewertung von Epikutantests muss die Möglichkeit von „falsch positiven“ und „falsch negativen“ Reaktionen in Erwägung gezogen werden. Bei der falsch positiven Reaktion handelt es sich um eine Hautreaktion ohne Vorliegen einer Kontaktallergie. Diese kann aus der Verwendung von Allergenen in zu hoher bzw. irritativer Konzentration resultieren, aber auch durch wiederholte Tests an derselben Stelle innerhalb kurzer Zeit (Belsito 2000).

Beschrieben ist auch ein sog. angry back/ excited skin-Syndrom. Dabei haben mehrere chemisch nicht verwandte Substanzen in direkter Nachbarschaft einen reaktionsfördernden Effekt und führen so zu jeweils heftigen positiven Reaktion beim Epikutantest (Bruynzeel und Maibach 1986). Bei einem Test mit nur einer Substanz wäre keine Reaktion zu beobachten (Schnuch et al. 2001).

Falsch negative Bewertungen liegen vor, wenn trotz bekannter Allergie keine positive Hautreaktion auftritt. Bei Verwendung einer zu niedrigen Testkonzentration, zu kurzer Applikationsdauer oder zu frühem Ablesezeitpunkt kann es $\mathrm{zu}$ falsch negativen Tests kommen. Auch die Applikationsart, Auswahl des Testvehikels und des Applikationsortes können eine falsch negative Reaktion als Konsequenz haben (Belsito 2000). Eine Bestrahlung mit UV-B oder eine PUVA-Therapie kann eine allergische Hautreaktion unterdrücken. Genauso eine Behandlung mit Kortikoiden oder Immunsuppressiva. Bei Patienten in höherem Alter muss zudem eine insgesamt abgeschwächte Immunantwort berücksichtigt werden (Belsito 2000).

\subsection{MOAHLFA-Index}

Der MOAHLFA Index steht für die englischen Abkürzungen von male, occupational, atopy,

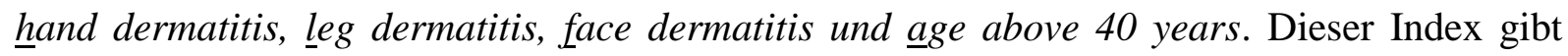
Informationen über demographische und klinische Charakteristika der getesteten Patienten. Dadurch können zeitliche Verläufe erfasst werden und in Relation zu den ermittelten Sensibilisierungsquoten gesetzt werden. Eine Vergleichbarkeit der Merkmale zwischen 
verschiedenen Studienzentren wird erleichtert und trägt zu einer Standardisierung der Ergebnisse bei (Uter et al. 1999).

\subsection{Allergene der Standardreihe und andere Testreihen}

Angesichts von über 3700 potentiellen Allergenen (Bruze et al. 1999) ist eine Auswahl der zu testenden Stoffe aus organisatorischen, kostentechnischen und klinisch relevanten Gründen nicht einfach. Die DKG gibt auf Grundlage des aktuellen wissenschaftlichen Erkenntnisstandes Empfehlungen über regelmäßig zu testende Allergene in der sogenannten Standardreihe aus. Die Standardreihe beinhaltet 29 Allergene, die bei routinemäßiger Testung an einem unselektiertem Kollektiv von Patienten, für welche die Indikation zum Epikutantest gestellt wurde, eine Reaktionsquote von $>1 \%$ beobachtet wurde (Schnuch et al. 2005c). Die Sensibilisierungsquoten der Standardreihe der DKG (Worm et al. 2005), auf die im Einzelnen in dieser Arbeit nicht eingegangen wird, sehen wie folgt aus:

\begin{tabular}{|c|c|c|c|}
\hline Substanz & Konz. in \% / Veh. & $\%$ pos. & Anzahl (=n) \\
\hline Nickel-(II-)sulfat, $6 * \mathrm{H} 2 \mathrm{O}$ & 5 / Vas. & 14,6 & 30671 \\
\hline Perubalsam & 25 / Vas. & 9,2 & 30999 \\
\hline Duftstoffmix & $8 /$ Vas. & 8,8 & 30933 \\
\hline Kobalt-(II-)chlorid, 6*H2O & $1 /$ Vas. & 6,4 & 30935 \\
\hline Kaliumdichromat & 0,5 / Vas. & 5,5 & 30982 \\
\hline Kolophonium & $20 /$ Vas. & 4,7 & 30999 \\
\hline p-Phenylendiamin (freie Base) & $1 /$ Vas. & 4,0 & 30740 \\
\hline Wollwachsalkohole & 30 / Vas. & 4,3 & 31017 \\
\hline Dibromdicyanobutan (Phenoxyehtl.) & 1/Vas. & 4,1 & 30630 \\
\hline Kompositenmix & $6 /$ Vas. & 3,2 & 30584 \\
\hline Quecksilber-(II-)amidchlorid & $1 /$ Vas. & 2,7 & 31040 \\
\hline diphemix & $1 /$ Vas. & 2,6 & 30977 \\
\hline HMPCC (Lyral) & $5 /$ Vas. & 2,3 & 25676 \\
\hline Neomycinsulfat & 20 / Vas. & 2,3 & 31012 \\
\hline Propolis & $10 /$ Vas. & 2,3 & 31029 \\
\hline (Chlor-)Methylisothiazolinon & $0,01 /$ Aqua & 2,2 & 31058 \\
\hline Terpentin & $10 /$ Vas. & 1,9 & 31019 \\
\hline Formaldehyd & 1/ Aqua & 1,7 & 31045 \\
\hline Bufexamac & 5 / Vas. & 1,5 & 31033 \\
\hline Dispersionsmix Blau 124/106 & $1 /$ Vas. & 1,5 & 22485 \\
\hline Benzocain & $5 /$ Vas. & 1,5 & 31025 \\
\hline Epoxidharz & $1 /$ Vas. & 1,4 & 30932 \\
\hline Cetylstearylalkohol & 20 / Vas. & 1,3 & 31058 \\
\hline p-tert-Butylphenol-Formaldehydharz & $1 /$ Vas. & 1,2 & 31040 \\
\hline IPPDd & $0,1 /$ Vas. & 0,8 & 31047 \\
\hline Mercaptomix ohne MBT & $1 /$ Vas. & 0,8 & 30959 \\
\hline Mercaptobenzothiazol & $2 /$ Vas. & 0,8 & 30705 \\
\hline Zink-diethyldithiocarbamat & $1 /$ Vas. & 0,6 & 31066 \\
\hline
\end{tabular}


Des Weiteren können Allergene in verschiedene Stoffreihen eingeteilt und getestet werden: Nach Verwendung (z. B. Kühlschmierstoffe, Duftstoffe, Antibiotika etc.), chemischen Eigenschaften (z.B. Kortikoide, Anästhetika) oder bei entsprechendem klinischem Verdacht parallel zur Standardreihe in Ergänzungsreihen (z.B. Dentalreihe, Friseurreihe). Durch die systematische Anwendung der ergänzenden Tests können Ursachen für eine Vielzahl von Kontaktallergien aufgedeckt werden. Diese Testreihen bedürfen einer stetigen Überwachung, die weltweit von verschiedenen Institutionen durchgeführt wird. Das Überwachungssystem im deutschsprachigen Raum ist der IVDK mit 40 teilnehmenden Instituten in Deutschland, der Schweiz und Österreich (Aberer et al. 2003). Die Funktionen dieser Überwachung sind vielfältig und liegen im Nachweis der Persistenz eines Problems, Relativierung eines vermuteten Problems, Identifizierung neuer Probleme, Erkennung von Trends und Nachweis des Erfolges durch Interventionen (Schnuch et al. 2005c).

Die Dermatologische Abteilung der Universitätsklinik Göttingen ist Gründungsmitglied des IVDK und auch die Zentrale des IVDK ist an die Klinik angeschlossen. In regelmäßigen monatlichen Abständen laufen aktuelle Daten aus allen beteiligten Zentren hier zusammen und werden regelmäßig online auf ihrer Homepage (www.ivdk.org) publiziert.

Darüber hinaus werden in den verschiedenen Zentren abhängig von lokalen Gegebenheiten sowie anamnestischen oder klinischen Verdachtsmomenten Testungen von nicht standardmäßig untersuchten Allergenen durchgeführt. Diese Testungen können im Rahmen von multizentrischen oder lokalen Untersuchungen erfolgen.

In der Vergangenheit haben die Auswertungen dieser zusätzlicher Testungen regelmäßig zu neuen Erkenntnissen und Trends geführt, die zu Anpassungen der Standardreihe bzw. Stoffreihen geführt haben.

\subsection{Anforderungen zur Aufnahme von Allergenen in die Standardreihe}

Angesichts einer großen Anzahl an Allergenen (s.o.) ist es unmöglich, alle verdächtigen Stoffe zu testen. Gleichzeitig ist bekannt, dass eine vergleichsweise kleine Anzahl an Substanzen für die Mehrzahl der Sensibilisierungen verantwortlich ist (Bruze et al. 1999). Diese relevanten Substanzen zu erkennen und mittels regelmäßigen Testungen in die Standardreihe aufzunehmen, ist die Aufgabe vieler nationaler und internationaler Organisationen. Es wird angenommen, dass mittels Standardreihe circa $70 \%$ bis $80 \%$ aller Kontaktallergien aufdeckt werden können (Bruze et al. 1999). 
Aus praktischen Gründen sollte die Anzahl an Allergenen in der Standardreihe zwischen 2030 nicht übersteigen. Weitere verdächtige Substanzen können bei entsprechender Indikation in Zusatztests und Zusatztestreihen untersucht werden.

Die European Society of Contact Dermatitis hat folgende Anforderungen für eine Substanz definiert, um in die Standardreihe aufgenommen zu werden (Bruze et al. 1999):

- Prävalenz mit einer Sensibilisierungsquote von mehr als $1 \%$ bei routinemäßig, $\mathrm{d}$. $\mathrm{h}$. fortlaufend getesteten Patienten

- verbreitetes Vorkommen

- verlässliche Epikutantestresultate

- hohes Maß an klinischer Relevanz

- minimale unerwünschte Effekte, insbesondere aktive Sensibilisierungen

Für die meisten Substanzen fehlen objektive Daten und Studien bezüglich der klinischen Relevanz und dies stellt eine besondere Schwierigkeit dar. Optimalerweise müsste die Relevanz im Vergleich zur reinen Sensibilisierungsquote höher gewichtet werden, jedoch ist es schwierig, diesbezüglich verlässliche und objektive Daten zu erheben. In manchen Fällen, in denen die Relevanz bekannt ist, kann diese Information ausschlaggebend für eine Aufnahme in die Standardreihe sein. Damit die Standardreihe nicht zu groß wird, muss regelmäßig evaluiert werden, ob Testungen mit einer Substanz weiterhin gerechtfertigt sind (Aberer et al. 2003). 


\subsection{Hitliste}

Als „Hitliste“ wird die Zusammenstellung der Sensibilisierungsraten einzelner Allergene in absteigender Häufigkeit bezeichnet, die in einem Patientenkollektiv mit dem Epikutantest ermittelt wurde (Schnuch et al. 2002b) und der Vereinfachung der Überwachung der Quoten dient. Beispielhaft sind hier die zehn häufigsten Allergene, die in Göttingen zwischen 1996 bis 2004 zu einer positiven Reaktion am Tag 3 geführt haben, aufgeführt $(n=3466)$ :

\begin{tabular}{|l|l|l|}
\hline Substanz & Konz. in \% / Vehikel & Pos. Reaktionen \\
\hline Nickel (II)-sulfat 6*H2O & $5 /$ Vas. & 411 \\
\hline Duftstoff-Mix & $8 /$ Vas. & 251 \\
\hline Perubalsam & $25 /$ Vas. & 221 \\
\hline Kolophonium & $20 /$ Vas. & 132 \\
\hline Thiomersal & $0,1 /$ Vas. & 126 \\
\hline Kobalt (II)-chlorid, 6*H2O & $1 /$ Vas. & 123 \\
\hline Kaliumdichromat & $0,5 /$ Vas. & 114 \\
\hline p-Phenylendiamin (Freie Base) & $1 /$ Vas. & 112 \\
\hline Wollwachsalkohole & $30 /$ Vas. & 94 \\
\hline Dibromdicyanobutan & $0,30 /$ Vas. & 91 \\
\hline
\end{tabular}

Tab.2: Die 10 häufigsten positiven Reaktionen ( $\mathrm{n}=3466)$ der Göttinger Hitliste zwischen 1996-2004 (siehe Anhang)

Die komplette Liste ist im Anhang (Seite 98-104) aufgeführt, jedoch stehen die Allergene in dieser Arbeit nicht einzeln zur Diskussion.

\subsection{Fragestellung}

Anhand von Epikutantestungen von möglichen Allergenen, die parallel zur Standardreihe im Zeitraum 1996 bis 2004 in der Dermatologischen Klinik der Universitätsmedizin in Göttingen getestet wurden, und den daraus erhobenen Daten soll in dieser retrospektiven Studie folgenden Fragen nachgegangen werden:

1. Wann werden die Testsubstanzen eingesetzt?

2. Welche Reaktionsquoten wurden in Göttingen beobachtet?

3. Wie stellen sich diese Reaktionsquoten im Vergleich mit der Literatur dar?

4. Ist es sinnvoll, parallel zur Standardreihe Epikutantests durchzuführen?

5. Welche Substanzen sollten parallel zur Standardreihe getestet werden und in welchem Umfang?

6. Sollten auf Grund der Ergebnisse bestimmte Substanzen in die Standardreihe aufgenommen werden? 


\section{Material und Methoden}

In der Dermatologischen Klinik der Universitätsmedizin in Göttingen erfolgten im Zeitraum zwischen 1996 und 2004 Epikutantestungen mit dem Finn-Chambers auf Scanpor®-System der Firma Hermal in Reinbek. Die routinemäßige Expositionsdauer des Epikutantests betrug 48 Stunden. Die Ablesung der Tests erfolgte nach 48 und 72 Stunden. Zur Auswertung wurden die Testreaktionen an Tag 3 herangezogen. Spätablesungen wurden nach 4, 5 und/oder 6 Tagen vorgenommen.

Die Beurteilungen der Epikutantestungen erfolgten nach den Richtlinien der Deutschen Kontaktallergiegruppe (DKG). Sie wurden von erfahrenen Ärzten der Allergologie Göttingen durchgeführt. Es wurde darauf geachtet, dass der einzelne Patient möglichst immer von derselben Person beurteilt wurde.

Vor der Untersuchung wurde ein standardisierter Anamnesefragebogen ausgefüllt (siehe Seiten 78 und 79). Die Datenauswertung erfolgte in der Zentrale des IVDK an der Universitäts- Hautklinik Göttingen unter Verwendung des statistischen Auswertungsprogrammes SAS (Version 9.2, SAS Inc., Cary, North Carolina, USA 2004). 


\section{Ergebnisse}

\subsection{Analyse der Patientendaten (s. Anhang, ab Seite 71)}

Insgesamt untersuchten wir 3466 Patienten mittels Epikutantest. Dabei handelte es sich im Allgemeinen um ambulante Patienten der Dermatologischen Abteilung. Einige wurden im Testzeitraum mehrfach, d. h. konsekutiv hintereinander, getestet. Sie wurden jedoch in der Auswertung als ein Patient gezählt.

Die Mehrzahl der Patienten war weiblich $(60.8 \%)$ und hatte ein Alter von mehr als 40 Jahren $(56,2 \%)$ mit einem Median von 43,5 Jahren.

Bei knapp einem Drittel der Patienten $(31,4 \%)$ wurde ein allergisches

Kontaktekzem (AKE) diagnostiziert.

Die Indikation zur Epikutantestung wurde überwiegend bei Verdacht auf

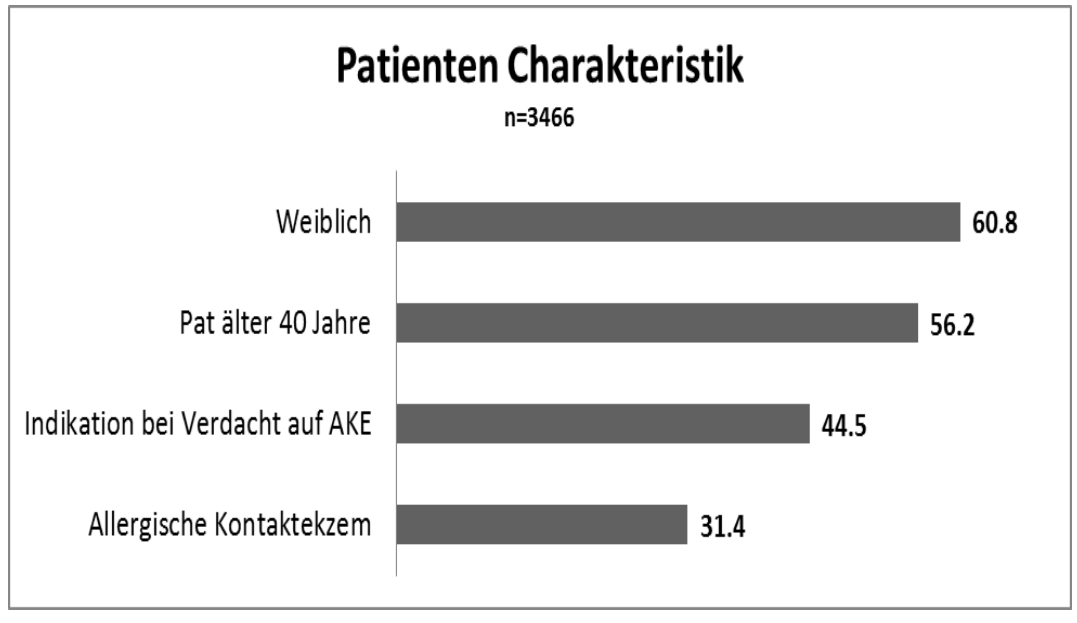
$(44,5 \%)$ oder zum Ausschluss einer allergischen Kontaktdermatitis (45.3\%) gestellt.

Die klinischen Lokalisationsorte des

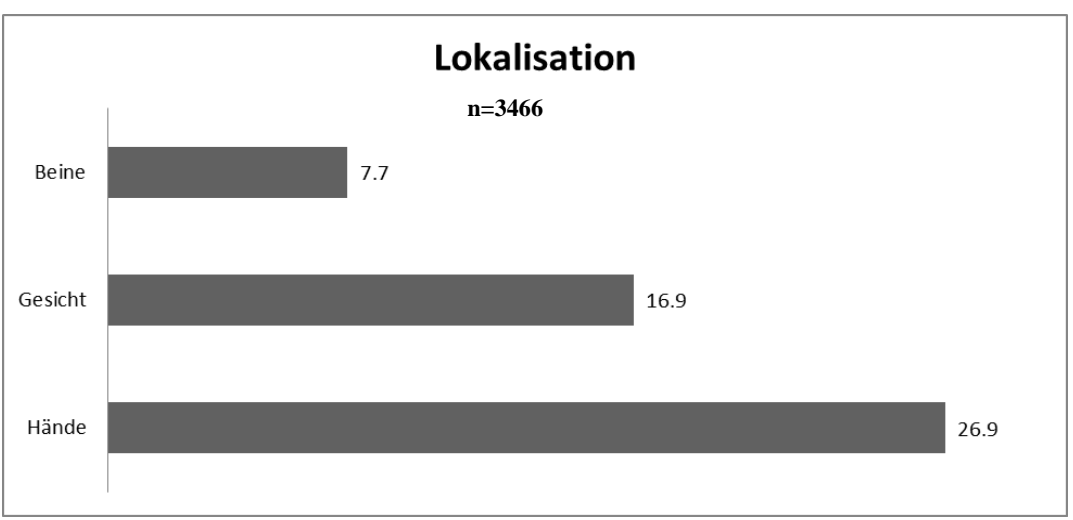

Die Berufsgruppe der Rentner war mit $21,0 \%$ am häufigsten vertreten, gefolgt von Hausfrauen $(10,8 \%)$ und Bürofachkräften $(5,7 \%)$, wobei Tätigkeiten mit unbestimmter Exposition mit 33,6\% am häufigsten waren.

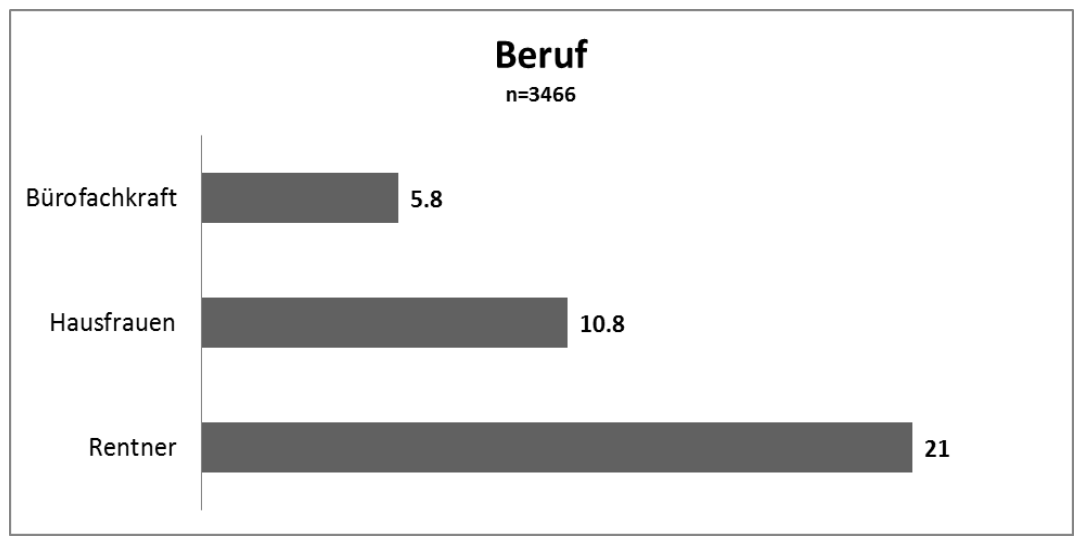


Bei der Anamnese gab circa jeder fünfte Patient (19,8\%) an, früher oder aktuell eine atopische Dermatitis zu haben.

„Heuschnupfen“ (18,5\%) und „Asthma“ $(8,4 \%)$ wurden weiterhin häufig angegeben.

Bei etwa jedem dritten Untersuchten $(34,8 \%)$ wurde der Kontakt zu

Allergenen in äusserlich anwendbaren Medikamenten sowie in Kosmetika und Cremes als Quelle in etwa einem Viertel der Fälle $(24,5 \%)$ vermutet, gefolgt von Seifen und Shampoos $(9,4 \%)$, Handschuhe (6,7\%) und Parfüms $(6,6 \%)$.

Während drei von vier Patienten $(74,7 \%)$ keine oder unbekannte Kofaktoren eines allergischen Kontaktekzems angaben, gab es bei etwas mehr als jedem fünften Patienten $(22,1 \%)$ anamnestische Hinweise.

Am häufigsten vertreten waren hierbei Arbeiten in Nässe $(21,4 \%)$, chronisch venöse Insuffizienz $(17,3 \%)$ und Arbeiten im okklusiven Milieu (14,4\%).
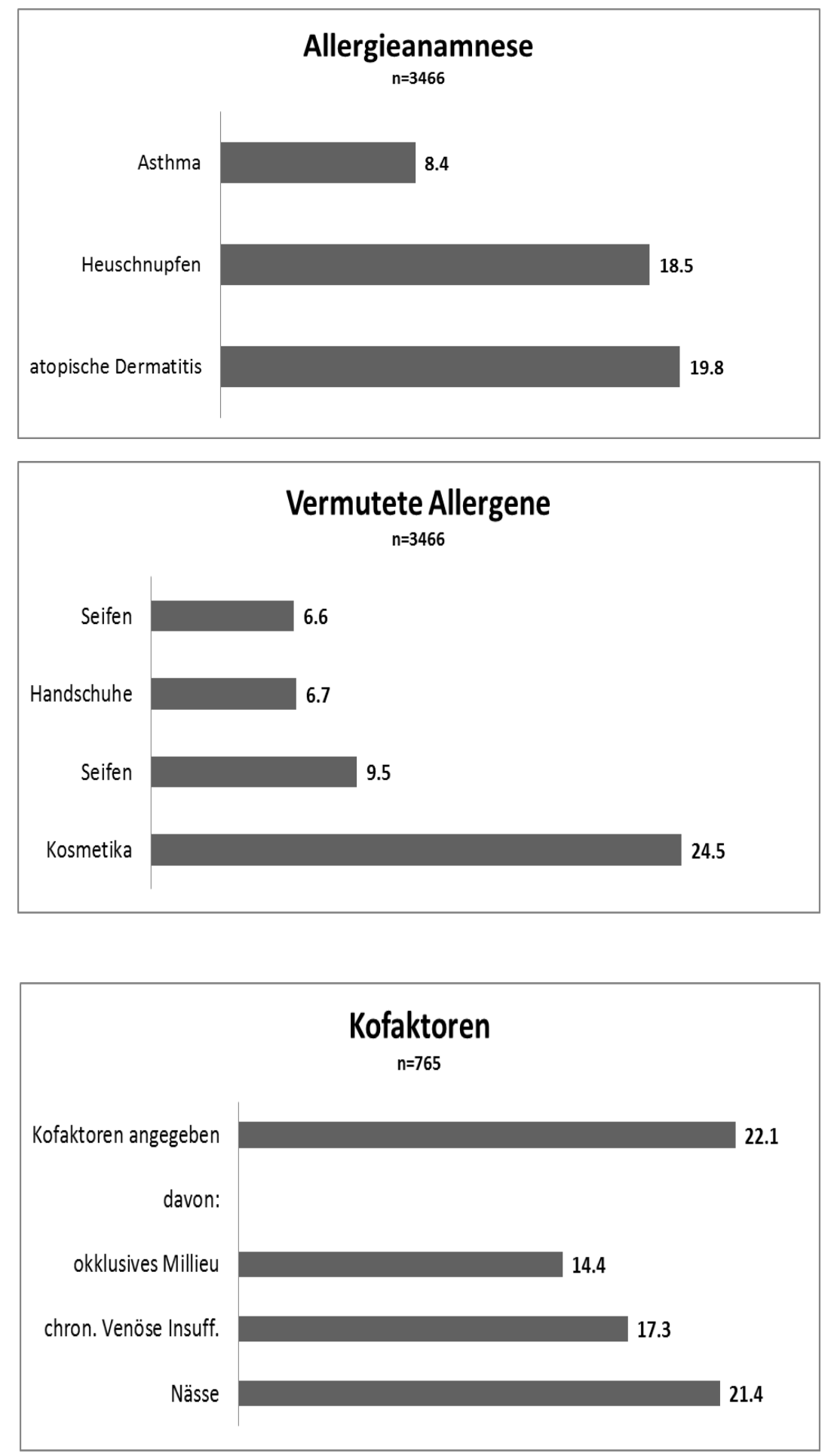

\subsection{Testsubstanzen und Ergebnisse der Epikutantests}

Die Epikutantestungen wurden mit Testsubstanzen aus der jeweils aktuellen DKG Standardreihe, dem sogenannten Block 1, durchgeführt. Parallel dazu wurde eine Auswahl an weiteren 45 Substanzen in vier weiteren Ergänzungsreihen, den sogenannten Blöcken 401, 707,713 und 714, getestet. 
Die Auswahl der Testsubstanzen erfolgte im Rahmen von für Göttingen spezifischen Verdachtsmomenten oder im Rahmen von multizentrischen Untersuchungen des IVDK.

Die Blockreihe Nummer 1 enthielt die Standardreihe der DKG:

\begin{tabular}{|c|c|c|}
\hline Substanz & Test Konz. in \% & Vehikel \\
\hline (Chlor)-Methylisothiazolinon (MCI/MI) & 0.01 & Aqua \\
\hline Benzocain (Ethylaminobenzoat) & 5.00 & Vas \\
\hline Bronopol (2-Brom-2-nitropropan-1,3-diol) & 0.50 & Vas \\
\hline Bufexamac & 5.00 & Vas \\
\hline Cetylstearylalkohol & 20.00 & Vas \\
\hline Clioquinol (Iodochlorhydroxyquin) & 5.00 & Vas \\
\hline Dibromdicyanobutan (Methyldibromo Glut.) & 0.30 & Vas \\
\hline Dibromdicyanobutan + 2-Phenoxyethanol & 0.50 & Vas \\
\hline Dibromdicyanobutan + 2-Phenoxyethanol & 1.00 & Vas \\
\hline Dispersions Mix Blau 124/106 & 1.00 & Vas \\
\hline Duftstoff-Mix & 8.00 & Vas \\
\hline Epoxidharz & 1.00 & Vas \\
\hline Formaldehyd & 1.00 & Aqua \\
\hline Kaliumdichromat & 0.50 & Vas \\
\hline Kobalt (II)-chlorid, $6 * \mathrm{H} 2 \mathrm{O}$ & 1.00 & Vas \\
\hline Kolophonium & 20.00 & Vas \\
\hline Kompositen-Mix & 6.00 & Vas \\
\hline Lyral & 5.00 & Vas \\
\hline Mercaptobenzothiazol & 2.00 & Vas \\
\hline Mercapto-Mix ohne MBT & 1.00 & Vas \\
\hline Neomycinsulfat & 20.00 & Vas \\
\hline Nickel (II)-sulfat $6 * \mathrm{H} 2 \mathrm{O}$ & 5.00 & Vas \\
\hline N-Isopropyl-N'-phenyl-p-phenylendiamin & 0.10 & Vas \\
\hline Paraben-Mix & 16.00 & Vas \\
\hline Perubalsam & 25.00 & Vas \\
\hline p-Phenylendiamin (Freie Base)(CI 76060) & 1.00 & Vas \\
\hline Propolis & 10.00 & Vas \\
\hline p-tert.-Butylphenol-Formaldehydharz & 1.00 & Vas \\
\hline Quecksilber (II)-amid-chlorid & 1.00 & Vas \\
\hline Terpentin & 10.00 & Vas \\
\hline Thiomersal & 0.10 & Vas \\
\hline Thiuram Mix & 1.00 & Vas \\
\hline Vaselinum album & 100.00 & \\
\hline Wollwachsalkohole & 30.00 & Vas \\
\hline Zink-diethyldithiocarbamat & 1.00 & Vas \\
\hline
\end{tabular}

Tab. 3: Blockreihe 1 Göttingen 
Die Blockreihe Nummer 401(Ergänzung Standardreihe) enthielt:

\begin{tabular}{|c|c|c|}
\hline Substanz & Konz. in \% & Vehikel \\
\hline 1,3-Diphenylguanidin (DPG) & 1.00 & Vas \\
\hline 2-Phenoxyethanol & 1.00 & Vas \\
\hline Amalgam (mit Zink) & 5.00 & Vas \\
\hline Amalgam (ohne Zink) & 5.00 & Vas \\
\hline Cain-Mix 1 (Procain 1\% und Dibucain 1\%) & 7.00 & Vas \\
\hline Carba-Mix & 3.00 & Vas \\
\hline Clobetasol-17-propionat & 0.25 & Vas \\
\hline Dibromdicyanobutan (Tektamer 38) & 0.10 & Vas \\
\hline Dibromdicyanobutan (Tektamer 38) & 0.30 & Vas \\
\hline Dibromdicyanobutan + 2-Phenoxyethanol & 0.50 & Vas \\
\hline Dispers Blau 124 & 1.00 & Vas \\
\hline Glutaraldehyd & 0.30 & Vas \\
\hline Hg.-Präcipitatsalbe & 1.00 & Vas \\
\hline Hydrocortison-17-butyrat & 0.10 & Vas \\
\hline Imidazolidinylharnstoff (Germall 115) & 1.00 & Vas \\
\hline Incidin plus & 0.10 & Vas \\
\hline Iodpropinylbutylcarbamat & 0.10 & Vas \\
\hline Iodpropinylbutylcarbamat & 0.50 & Vas \\
\hline Kolophonium-Mix II & 15.00 & Vas \\
\hline Kompositen-Mix & 6.00 & Vas \\
\hline Latex-Handschuhextrakt 4\%ige Pricklösg. & 100.00 & \\
\hline Lyral & 5.00 & Vas \\
\hline Mercaptobenzothiazol & 2.00 & Vas \\
\hline Myristylalkohol & 10.00 & Vas \\
\hline Natriumlaurylsulfat & 0.25 & Vas \\
\hline Natriumlaurylsulfat & 0.50 & Vas \\
\hline Natriumlaurylsulfat & 1.00 & Vas \\
\hline p-Aminomethylbenzolsulfonamid (Mafenid) & 10.00 & Vas \\
\hline Poly(hexamethylendiguanid)-HCl & 2.50 & Aqua \\
\hline Poly(hexamethylendiguanid)-HCl & 2.50 & Vas \\
\hline Propolis & 10.00 & Vas \\
\hline Propylenglycol & 5.00 & Vas \\
\hline Propylenglycol & 20.00 & Aqua \\
\hline p-Toluylendiamin (freie Base) & 1.00 & Vas \\
\hline Quaternium 15 & 1.00 & Vas \\
\hline Sesquiterpenlactone Mix & 0.10 & Vas \\
\hline Tixocortol-pivalat & 1.00 & Vas \\
\hline Unguentum leniens DAB 10 & 100.00 & \\
\hline Zinkdibenzyldithiocarbamat & 1.00 & Vas \\
\hline Zinkdibutyldithiocarbamat & 1.00 & Vas \\
\hline Zinkdibutyldithiocarbamat & 3.00 & Vas \\
\hline
\end{tabular}

Tab. 4: Blockreihe 401 Göttingen 
Die Blockreihen 707 und 714 (Anhang IV Standardreihe) enthielten Teebaumöl:

\begin{tabular}{|l|l|l|}
\hline Substanz & Konz. in \% & Vehikel \\
\hline Teebaumöl & 5.00 & DEP \\
\hline Teebaumöl & 20.00 & Oli \\
\hline
\end{tabular}

Tab. 5: Blockreihe 707 und 714 in Göttingen

Die Blockreihe 713 (Anhang IV Standardreihe) enthielt folgende Substanzen:

\begin{tabular}{|l|l|l|}
\hline Substanz & Konz. in \% & Vehikel \\
\hline 2-Mercaptobenzimidazol & 1.00 & Vas. \\
\hline 7-Ethylbicyclooxazolidin (Bioban CS 1246 ) & 1.00 & Vas. \\
\hline Bronidox L (5-Brom-5-nitro-1,3-dioxan) & 0.25 & Vas. \\
\hline Bronidox L (5-Brom-5-nitro-1,3-dioxan) & 0.50 & Vas. \\
\hline Bufexamac & 5.00 & Vas. \\
\hline Carboxymethylcellulose (Na-) & 2.00 & Vas. \\
\hline Dexpanthenol & 5.00 & Vas. \\
\hline Dimethylformamid & 0.10 & Vas. \\
\hline Dispersions Mix Blau 124/106 & 1.00 & Vas. \\
\hline Hexylzimtaldehyd & 2.00 & Vas. \\
\hline KKM 702 & 1.00 & Vas. \\
\hline $\begin{array}{l}\text { Lyral (4-(4-Hydroxy-4-methylpentyl)-3-cyclohexen-1- } \\
\text { carboxaldehyd) }\end{array}$ & 5.00 & Vas. \\
\hline Natriumdisulfit & & \\
\hline Prednicarbat & 1.00 & Vas. \\
\hline Prednicarbat & 0.10 & Vas. \\
\hline Tantalum & 0.25 & Vas. \\
\hline Tantalum & 0.10 & Vas. \\
\hline Tantalum-V-chloride & 1.00 & Vas. \\
\hline Tantalum-V-chloride & 0.10 & Vas. \\
\hline Toluolsulfonamid-Formaldehydharz & 1.00 & \\
\hline Unguentum Leniens & 10.00 & Vas. \\
\hline
\end{tabular}

Tab. 6: Blockreihe 713 in Göttingen 


\subsection{Ergebnisse der Epikutantests (1996 -2004)}

Nachfolgend sind die Substanzen (alphabetisch geordnet) und ihre Sensiblisierungsquoten aufgelistet, die wir in Göttingen parallel zur Standardreihe in den Ergänzungsblöcken getestet haben (die kompletten Tabellen finden sich im Anhang):

\begin{tabular}{|c|c|c|c|c|}
\hline Substanz & \% Konz. & Anzahl $(n=3466)$ & $\% \operatorname{pos}$ & ir \\
\hline 1,3-Diphenylguanidin (DPG) & 1.00 & 385 & 1.6 & 0 \\
\hline 2-Mercaptobenzimidazol & 1.00 & 231 & 0.4 & 0 \\
\hline 2-Phenoxyethanol & 1.00 & 554 & 0.5 & 0 \\
\hline 7-Ethylbicyclooxazolidin (Bioban CS 1246) & 1.00 & 256 & 0.4 & 0 \\
\hline Amalgam (mit Zink) & 5.00 & 2499 & 2.0 & 0 \\
\hline Amalgam (ohne Zink) & 5.00 & 140 & 1.4 & 0 \\
\hline Bronidox L (5-Brom-5-nitro-1,3-dioxan) & 0.25 & 2720 & 0.5 & 0 \\
\hline Bronidox L (5-Brom-5-nitro-1,3-dioxan) & 0.50 & 212 & 5.7 & 1 \\
\hline Bufexamac & 5.00 & 1536 & 2.1 & 0 \\
\hline Cain-Mix 1 (Procain 1\% und Dibucain 1\%) & 7.00 & 611 & 2.3 & 0 \\
\hline Carba-Mix & 3.00 & 388 & 3.1 & 1 \\
\hline Carboxymethylcellulose (Na-) & 2.00 & 2385 & 0 & 0 \\
\hline Clobetasol-17-propionat & 0.25 & 3219 & 0.1 & 2 \\
\hline Dexpanthenol & 5.00 & 2126 & 1.0 & 0 \\
\hline Dibromdicyanobutan (Methyldibromo Glutamat) & 0.10 & 612 & 2.1 & 0 \\
\hline Dibromdicyanobutan (Methyldibromo Glutamat) & 0.30 & 1102 & 4.9 & 0 \\
\hline Dimethylformamid & 0.10 & 1953 & 0.1 & 0 \\
\hline Dispers Blau 124 & 1.00 & 612 & 0.7 & 0 \\
\hline Dispersions Mix Blau 124/106 & 1.00 & 62 & 1.6 & 0 \\
\hline Glutaraldehyd & 0.30 & 3218 & 0.3 & 55 \\
\hline Hexylzimtaldehyd & 2.00 & 792 & 0 & 0 \\
\hline Hg.-Präcipitatsalbe & 1.00 & 213 & 4.2 & 0 \\
\hline Hydrocortison-17-butyrat & 0.10 & 944 & 0.2 & 0 \\
\hline Imidazolidinylharnstoff (Germall 115) & 1.00 & 3214 & 0.5 & 0 \\
\hline Incidin plus & 0.10 & 263 & 0 & 0 \\
\hline Iodpropinylbutylcarbamat & 0.10 & 44 & 0 & 0 \\
\hline Iodpropinylbutylcarbamat & 0.50 & 44 & 0 & 0 \\
\hline KKM 702 (Abk?) & 1.00 & 2379 & 0 & 0 \\
\hline Kolophonium-Mix II & 15.00 & 179 & 1.7 & 0 \\
\hline Latex-Handschuhextrakt 4\%ige Pricklösg. & 100.00 & 178 & 0 & 0 \\
\hline $\begin{array}{l}\text { Lyral (4-(4-Hydroxy-4-methylpentyl)-3-cyclohexen-1- } \\
\text { carboxaldehyd) }\end{array}$ & 5.00 & 337 & 3.9 & 0 \\
\hline
\end{tabular}




\begin{tabular}{|c|c|c|c|c|}
\hline Substanz & \%Konz. & Anzahl (n=3466) & $\%$ pos & ir \\
\hline Mercaptobenzothiazol & 2.00 & 2044 & 0.8 & 0 \\
\hline Myristylalkohol & 10.00 & 28 & 7.1 & 0 \\
\hline Natriumdisulfit & 1.00 & 2635 & 1.3 & 0 \\
\hline Natriumlaurylsulfat & 0.25 & 1413 & 0.1 & 0 \\
\hline Natriumlaurylsulfat & 0.50 & 1413 & 0.1 & 6 \\
\hline Natriumlaurylsulfat & 1.00 & 1413 & 0.4 & 12 \\
\hline p-Aminomethylbenzolsulfonamid (Mafenid) & 10.00 & 611 & 0.7 & 0 \\
\hline Poly(hexamethylendiguanid)-HCl (Aqua) & 2.50 & 651 & 0.5 & 0 \\
\hline Poly(hexamethylendiguanid)-HCl (Vas.) & 2.50 & 464 & 0.2 & 0 \\
\hline Prednicarbat & 0.10 & 2385 & 0.3 & 0 \\
\hline Prednicarbat & 0.25 & 3200 & 0.3 & 0 \\
\hline Propolis & 10.00 & 1606 & 1.7 & 0 \\
\hline Propylenglycol & 5.00 & 3214 & 0.2 & 1 \\
\hline Propylenglycol & 20.00 & 611 & 2.0 & 0 \\
\hline p-Toluylendiamin (freie Base) & 1.00 & 612 & 1.6 & 2 \\
\hline Quaternium 15 & 1.00 & 2635 & 0.6 & 0 \\
\hline Sesquiterpenlactone Mix & 0.10 & 2629 & 1.1 & 0 \\
\hline Tantalum & 0.10 & 78 & 0 & 0 \\
\hline Tantalum & 1.00 & 370 & 0 & 0 \\
\hline Tantalum-V-chloride & 0.10 & 1947 & 0 & 0 \\
\hline Tantalum-V-chloride & 1.00 & 2783 & 0.3 & 0 \\
\hline Teebaumöl & 5.00 & 1171 & 0.7 & 0 \\
\hline Teebaumöl & 20.00 & 1719 & 3.7 & 1 \\
\hline Tixocortol-pivalat & 1.00 & 1641 & 0.9 & 0 \\
\hline Toluolsulfonamid-Formaldehydharz & 10.00 & 3174 & 0.2 & 0 \\
\hline Unguentum leniens DAB 10 & 100.00 & 1288 & 0.1 & 0 \\
\hline Zinkdibenzyldithiocarbamat & 1.00 & 554 & 0 & 0 \\
\hline Zink-dibutyldithiocarbamat & 1.00 & 3213 & 0.1 & 0 \\
\hline Zink-dibutyldithiocarbamat & 3.00 & 791 & 0 & 0 \\
\hline
\end{tabular}

Tab. 7: Ergebnisse der Ergänzungsreihen der Göttinger Substanzen 
Die Substanzen mit einer Sensibilisierungsquote von 1,0\% und höher waren folgende:

\begin{tabular}{|c|c|c|c|c|}
\hline Substanz & \%Konz. & Anzahl $(n=3466)$ & $\%$ pos & ir \\
\hline Myristylalkohol & 10.00 & 28 & 7.1 & 0 \\
\hline Bronidox L (5-Brom-5-nitro-1,3-dioxan) & 0.50 & 212 & 5.7 & 1 \\
\hline Dibromdicyanobutan (Methyldibromo Glutamat) & 0.30 & 1102 & 4.9 & 0 \\
\hline Hg.-Präcipitatsalbe & 1.00 & 213 & 4.2 & 0 \\
\hline $\begin{array}{l}\text { Lyral (4-(4-Hydroxy-4-methylpentyl)-3-cyclohexen-1- } \\
\text { carboxaldehyd) }\end{array}$ & 5.00 & 337 & 3.9 & 0 \\
\hline Teebaumöl & 20.00 & 1719 & 3.7 & 1 \\
\hline Carba-Mix & 3.00 & 388 & 3.1 & 1 \\
\hline Cain-Mix 1 (Procain 1\% und Dibucain 1\%) & 7.00 & 611 & 2.3 & 0 \\
\hline Bufexamac & 5.00 & 1536 & 2.1 & 0 \\
\hline Dibromdicyanobutan (Methyldibromo Glutamat) & 0.10 & 612 & 2.1 & 0 \\
\hline Amalgam (mit Zink) & 5.00 & 2499 & 2.0 & 0 \\
\hline Propylenglycol & 20.00 & 611 & 2.0 & 0 \\
\hline Kolophonium-Mix II & 15.00 & 179 & 1.7 & 0 \\
\hline Propolis & 10.00 & 1606 & 1.7 & 0 \\
\hline 1,3-Diphenylguanidin (DPG) & 1.00 & 385 & 1.6 & 0 \\
\hline Dispersions Mix Blau 124/106 & 1.00 & 62 & 1.6 & 0 \\
\hline p-Toluylendiamin (freie Base) & 1.00 & 612 & 1.6 & 2 \\
\hline Amalgam (ohne Zink) & 5.00 & 140 & 1.4 & 0 \\
\hline Natriumdisulfit & 1.00 & 2635 & 1.3 & 0 \\
\hline Sesquiterpenlactone Mix & 0.10 & 2629 & 1.1 & 0 \\
\hline Dexpanthenol & 5.00 & 2126 & 1.0 & 0 \\
\hline
\end{tabular}

Tab. 8: Ergebnisse der Ergänzungsreihen der Göttinger Substanzen mit $\geq 1 \%$ - Quote 


\subsection{Analyse des MOAHLFA-Index}

Die für den MOAHLFA-Index wichtigen Merkmale zeigten im zeitlichen Verlauf folgende Ergebnisse:

Im Allgemeinen wurde im Testzeitraum ein jährlicher Rückgang an Patienten von anfangs 544 in 1996 auf 307 in 2002 und 177 Patienten in 2004 verzeichnet. Dies geschah vornehmlich aus strukturellen Gründen.

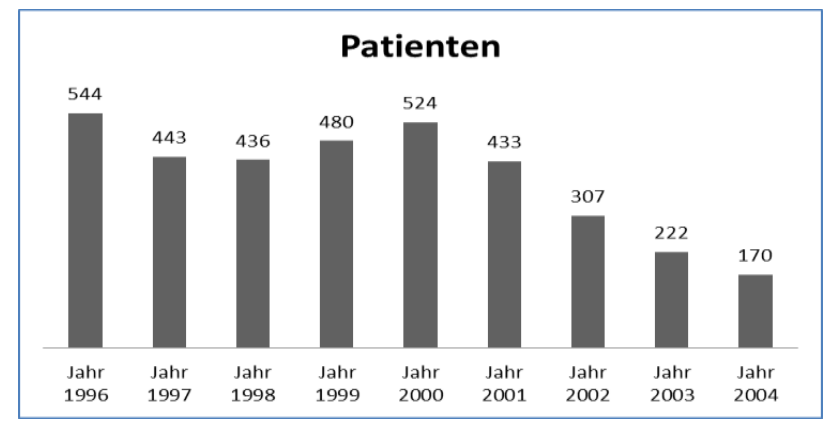

Das Merkmal „männlich“ zeigte einen anteiligen

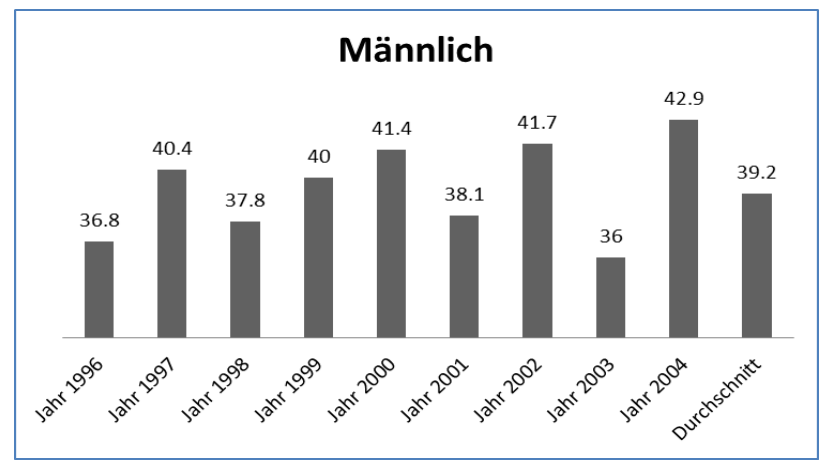

Die Berufsdermatitis zeigte zunächst eine Abnahme von 10,5\% in 1996 auf 7,2\% in 2003. Im Jahr 2004 konnte eine Zunahme auf 12,9\% beobachtet werden.

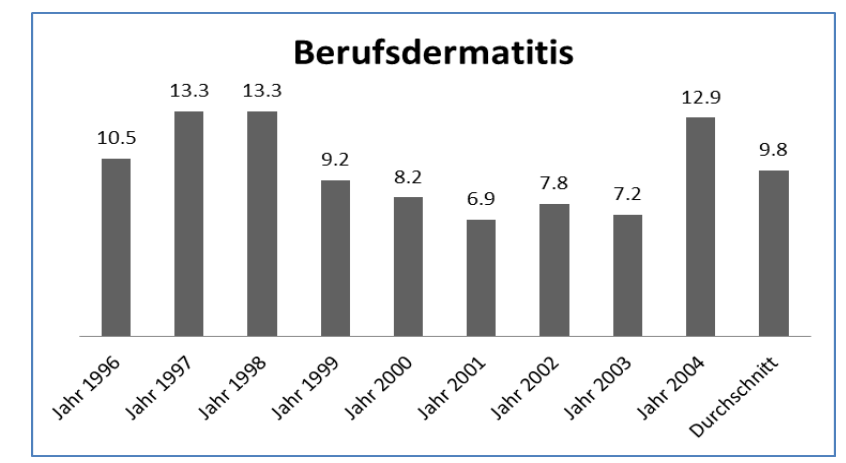

Die atopische Dermatitis zeigte eine wechselhafte Verteilung. 1996 betrug der Anteil 23,5\%. Dieser sank bis 2000 auf 12,2\%, stieg bis 2002 auf $32,6 \%$, um anschließend auf einen Anteil von $18,2 \%$ in 2004 zurück zu gehen.

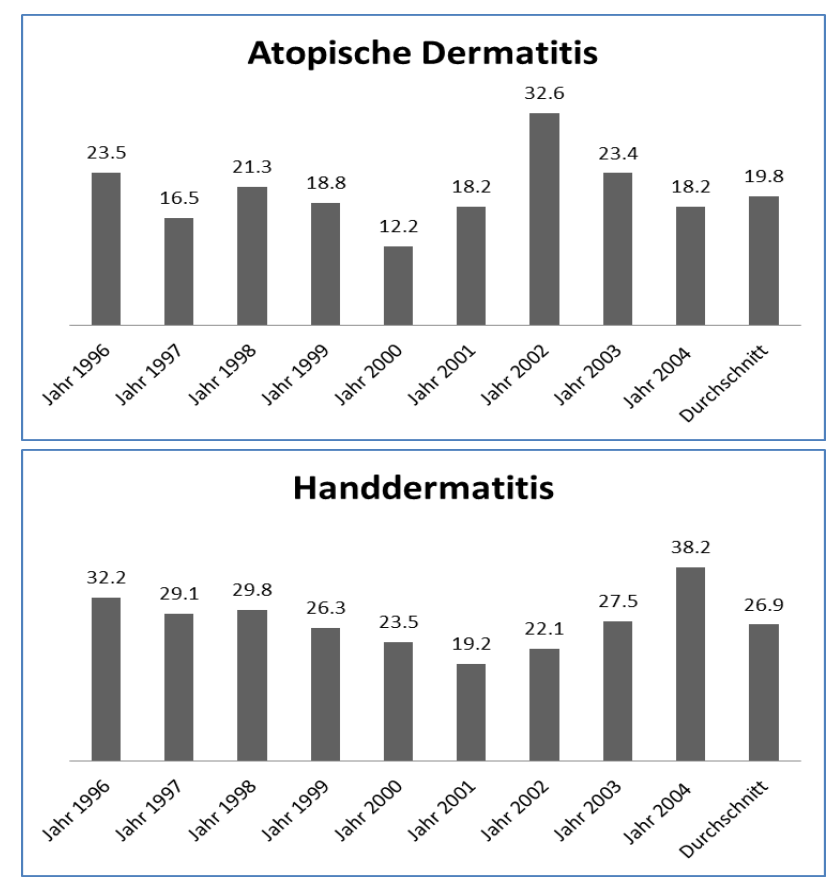


Bei der Beindermatitis wurde eine wechselhafte Verteilung mit von 5,9\% in 1996, 11,3\% in 2000, ein Absinken auf 3,5\% im folgenden Jahr und schließlich 11,2\% in 2004 beobachtet.

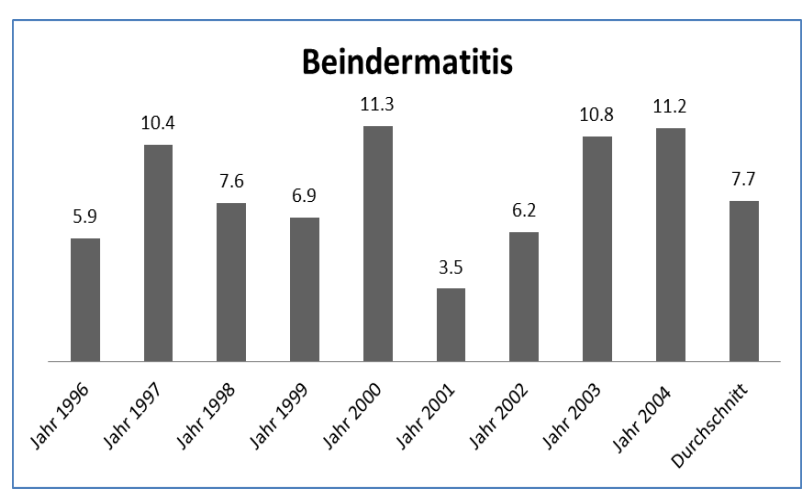

Die Gesichtsdermatitis zeigte einen relativ konstanten Verlauf mit einem Anteil von 17,3\% 1996 über 20,4\% 1999 auf 14,1\% 2004.
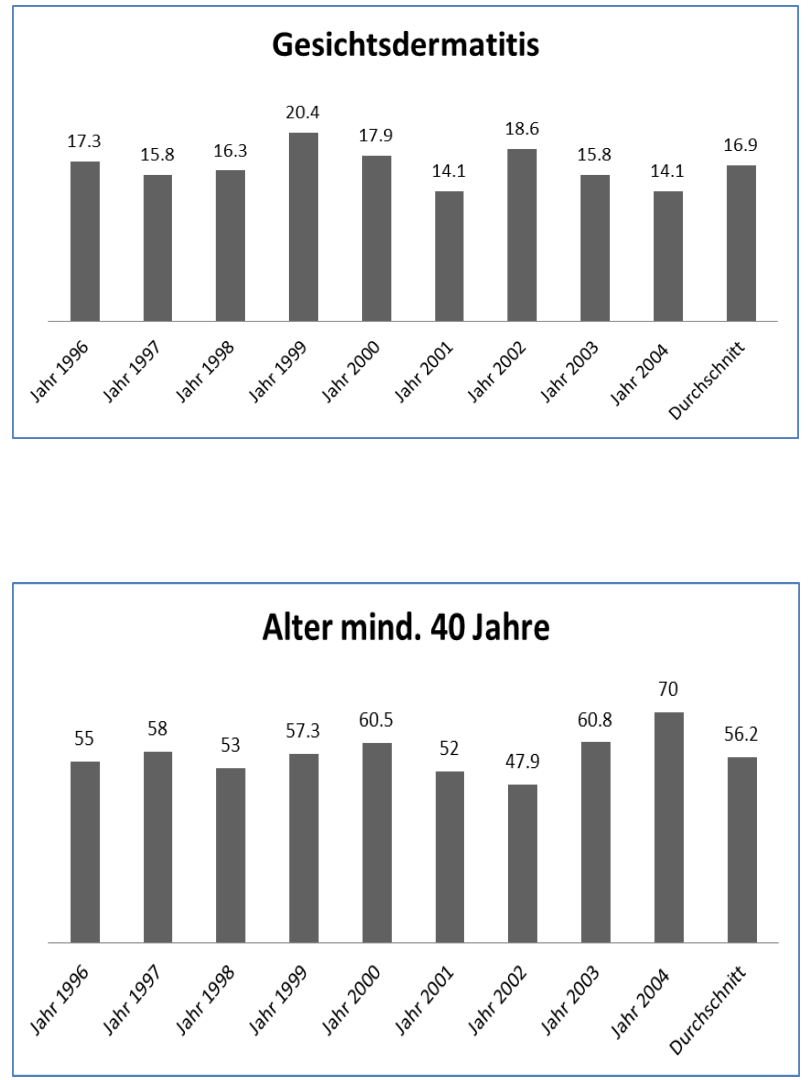

Das Alter von mindestens 40 Jahren zeigte einen weitgehend konstanten Verlauf mit einem Anteil von $55 \%$ bis $57,3 \%$ im Zeitraum von 1996 bis 1999. Ab dem Jahre 2000 konnte ein Anstieg auf zuletzt 70,0\% im Jahre 2004 beobachtet werden. 


\section{Diskussion}

\subsection{Testpopulation}

Anhand des MOAHLFA-Index kann man für die Göttinger Testpopulation feststellen, dass sie mehrheitlich weiblichen Geschlechts war, mit einem Alter von über 40 Jahre. Ungefähr jeder fünfte hatte eine atopische Dermatitis. Diese Verteilung ist vergleichbar mit der Gesamtpopulation des IVDK vom Zeitraum 1999 bis 2006 (Uter et al. 2008). Die größten Unterschiede lassen sich in Göttingen in einem geringeren Anteil an Patienten mit Berufsdermatosen und dem Merkmal Bein Dermatitis erkennen (Uter et al. 2008). Dadurch könnten niedrigere Sensibilisierungsraten auf Stoffe erklärt werden, mit denen Patienten im Rahmen einer beruflichen Exposition in Kontakt kommen oder die bevorzugt zur Behandlung eines Unterschenkelekzems eingesetzt werden. Zum Vergleich sind weitere europäische Daten aus Österreich, Schweiz und Italien in Tabelle 8 aufgeführt (Uter et al. 2012).

\begin{tabular}{ll|llllll}
\hline Merkmal & & Göttingen (\%) & IVDK (\%) & IVDK (\%) & Österreich (\%) & Schweiz (\%) & Italien (\%) \\
& & $1996-2004$ & $1999-2006$ & $2007-2008$ & $2007-2008$ & $2007-2008$ & $2007-2008$ \\
\hline Männlich & M & 39.2 & 37.8 & 39 & 34 & 40 & 34 \\
Berufsdermatose & O & 9.8 & 16.0 & 22 & 16 & 17 & 10 \\
Atop. Dermatitis & A & 19.8 & 17.0 & 16 & 17 & 17 & 10 \\
Hand-Dermatitis & H & 26.9 & 28.3 & 31 & 24 & 31 & 27 \\
Bein-Dermatitis & L & 7.7 & 11.1 & 8 & 9 & 7 & 9 \\
Face-Dermatitis & F & 16.9 & 13.7 & 17 & 14 & 17 & 17 \\
Alter mind. 40 & A & 56.2 & 65.9 & 70 & 66 & 62 & 51 \\
\hline
\end{tabular}

Tab 8.: Vergleich MOAHLFA Index: Göttingen 1996-2004 und IVDK 1999-2006 (Uter, et al. 2008) und ESSCA 2007-2008 (Uter, et al. 2012) 


\subsection{Interpretation der Ergebnisse der Substanzen der Ergänzungsreihen}

\section{Amalgam}

CAS-Nr. 7439-97-6

Amalgam, eine metallische Quecksilberlegierung, hat sein Einsatzgebiet seit dem 19. Jahrhundert klassischerweise in der Füllungstherapie in der Zahnmedizin (Andersen et al. 1987). Seit der Einführung von Amalgamfüllungen gibt es kontroverse Diskussionen bezüglich Nebenwirkungen wie Quecksilberintoxikationen und Kontaktallergien (Fuchs 1994). Berichte über allergische Reaktionen waren höchstens als Einzelfälle, die bis zum Jahre 1905 zurückreichten, publiziert worden (Veron et al. 1986). In Rahmen multizentrischer Studien der DKG wurden zur Überprüfung der Situation in den 1990er Jahren quecksilberhaltige Stoffe parallel zur Standardreihe untersucht, unter anderem im Hinblick auf die Erhebung der Sensibilisierungsquoten und optimalen Testkonzentration. Dabei wurde in Göttingen Amalgam in einer 5\%-Konzentration getestet. Die Firma Hermal ® lieferte bis 1996 das getestete Amalgam ohne den zusätzlichen Inhaltsstoff Zink. Danach enthielt die Testsubstanz auch Zink, was keine allergologische Bedeutung hat, jedoch die entsprechende Unterscheidung in der Benennung bei den Testungen erklärt (s. Seite 20). Für Amalgam ohne Zink wurde in $1,4 \%(n=140)$ eine positive Reaktion und für Amalgam mit Zink in 2,0\% $(n=2499)$ eine positive Reaktion ermittelt. Im Vergleich dazu zeigte das Gesamtergebnis der DKG-Studie für Amalgam 5\% (Vas.) eine Quote von 7,6\% (n=367) und für Amalgam insgesamt in den Konzentrationen von 2-20\% (Vas.) eine Quote von 12\% (n=368) ( Mayenburg von et al. 1996). Gleichzeitig konnte in dieser Studie gezeigt werden, dass fast alle positiven Reaktionen auf Amalgam mittels Quecksilber (II)-amidchlorid als Marker einer Quecksilberallergie aufgedeckt werden können (Mayenburg von et al. 1996). Quecksilberamidchlorid kam daher als Marker in der Standardreihe routinemässig zum Einsatz. Da unspezifische Reaktionen, insbesondere unerwartete, ohne zusätzliche Testungen oft kaum zu interpretieren waren, und als Quelle der Quecksilberexposition am häufigsten Dentalwerkstoffe verdächtigt wurden, wurde diese Testsubstanz im Jahre 2005 aus der Standardreihe gestrichen und in die Testreihe "DKG Dentalmetalle" verlagert (Geier und Mahler 2016). 
p-Aminomethylbenzolsulfonamid (Mafenid)

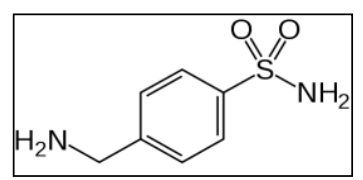

CAS Nr. 138-39-6 (v. Bruchhausen 1999)

p-Aminomethylbenzolsulfonamid, auch unter dem Namen Mafenid bekannt, ist ein topisch wirksames Sulfonamid (Chemotherapeutikum) und wurde seit den 1940er Jahren eingesetzt (Affee und Dressler 1969). In Verbänden und Salben, vor allem bei der BrandWundbehandlung, wurde Mafenid wegen guter bakteriostatischer Wirkung gegen Pseudomonas aeruginosa verwendet (Firoz et al. 2007). Zu diesem seit langem eingesetzten Medikament, welches u. a. in der Tiermedizin verwendet wird, lagen seit der Einführung vereinzelt Berichte von Kontaktdermatitiden vor (Fernandez et al. 1992) und (Sanz de Galdeano et al. 1993), so dass in Göttingen eine Testung parallel zur Standardreihe durchgeführt wurde. Dabei zeigten sich lediglich in $0,7 \%(n=611)$ positive Reaktionen. Auf Grund dieses Ergebnisses und fehlender Hinweise auf bedeutende Sensibilisierungsquoten in der Literatur (Firoz et al. 2007) sollte eine Testung von Mafenid nur gezielt erfolgen, wenn überhaupt.

\section{Bioban CS 1246}

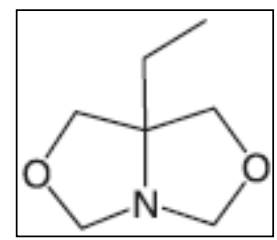

CAS Nr. 7747-35-5 (de Groot et al. 2010).

Bioban CS 1246 ist ein Formaldehyd-abspaltendes Biozid, welches als Konservierungsstoff und Korrosionsschutzmittel verwendet wird. Es kommt in der Metallverarbeitung sowie in Abdichtungen, Latexfarben und Klebstoffen zum Einsatz (Charles et al. 2005). Im Rahmen einer multizentrischen Studie des IVDK kam es in Göttingen zu einer Testung von Bioban CS 1246. Dabei zeigte sich in $0,4 \%(n=256)$ eine positive Reaktion. Eine frühere IVDK-Studie zeigte eine Rate von 1,1\% ( $\mathrm{n}=1111)$ (Geier et al. 1996a). In einer Studie in Dortmund wurde die Sensibilisierungsrate von Metallarbeitern mit Daten der Gesamtpopulation des IVDK verglichen. Dabei zeigten sich bei dieser ausgewählten Population in 2,0\% $(n=408)$ positive Reaktionen, während bei der IVDK-Gruppe, bestehend aus Personen mit verschiedenen Berufen, eine Sensibilisierungsrate von 0,8\% ( $\mathrm{n}=3389)$ beobachet wurde (Brinkmeier, et al. 2002). Die Ergebnisse dieser Daten ließen die Autoren vermuten, dass eine hohe Anzahl an 
Reaktionen irritativer Genese sein könnte, da die Reaktionen oft schwach positiv und nicht reproduzierbar waren (Brinkmeier et al. 2002). Die Vermutung wurde geäußert, dass hier abgespaltenes Formaldehyd, bekannt für irritative Eigenschaften, eine Rolle spielen könnte. Trotz der niedrigen Göttinger Rate von $0,4 \%$ ist eine gezielte Diagnostik bei klinischanamnestischen Verdachtsmomenten und bei beruflich Exponierten zu empfehlen. Bioban CS 1246 ist, auf Vorschlag der Arbeitsgruppe „Allergiediagnostik in der Metallbranche“, dem die DKG und die IVDK angehören, im Block der industriellen Biozide und Block der Kühlschmierstoffe vorhanden und kommt parallel zur Standardreihe regelmäßig zur Testung (Geier et al. 2000a). Die dort verzeichnete Sensibilisierungsrate von 2,5\% ( $n=215)$ scheint diesen Schritt zu rechtfertigen (Geier et al. 2004). In einer multizentrischen europäischen Untersuchung von selektierten Metallarbeitern lag die Rate bei 1,9\% (de Groot A et al. 2010).

\section{Bronidox L (5-Brom-5-Nitro-1,3-dioxan)}

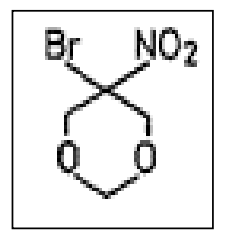

CAS Nr. 30007-47-7 (Potokar et al. 1976)

Bronidox L (5-Brom-5-Nitro-1,3-dioxan) wird wegen seinen antimikrobiellen Eigenschaften als Konservierungsmittel in abwaschbaren (sog. rinse-off) Kosmetika seit den 1970er Jahren eingesetzt (Matissek 1983). Zu diesen Produkten gehören Shampoos, Reinigungscremes und Schaumbäder. Aus Meerschweinchentests konnte man annehmen, dass diese Substanz ein Sensibilisierungspotential $(79 \%, n=24)$ hat (Gruvberger und Bruze 1997). Die Raten bei Patienten lagen, wobei unterschiedliche Vehikel untersucht wurden, für Bronidox L mit einer Konzentration von $0,25 \%$ in Ethanol bei $1,4 \%(n=1024)$ und in gleicher Konzentration in Vaseline als Vehikel bei 0,4\% ( $\mathrm{n}=1085)$ (Gruvberger und Bruze 1997).

In Göttingen wurden parallel zur Standradreihe Epikutantests durchgeführt. Hier wurden in den beiden getesteten Konzentrationen $(0,25 \%$ und $0,5 \%$ jeweils in Vaseline) positive Reaktionen in $0,5 \%(\mathrm{n}=2720)$ bzw. in $5,7 \%(\mathrm{n}=212)$ gesehen. Weitere Untersuchungen liegen nach unserer Kenntnis nicht vor.

Bronidox L scheint bislang nicht als ein sensibilisierender Stoff in Verdacht geraten zu sein. Jedoch sollten auf Grund der genannten Ergebnisse mit Nachweis von epidermalen Sensibilisierungen Epikutantests bei anamnestischem Verdacht auf eine Kosmetikaallergie durchgeführt werden. Es gilt jedoch, die Testkonzentration und das Testvehikel zu optimieren. Ethanol-Lösungen scheinen Konservierungsmittel abzubauen (Gruvberger und 
Bruze 1997). Bei unseren Epikutantests zeigte Bronidox L in 0,5\% Konzentration in Vaseline die höchste Detektionsrate zeigte, wobei nicht auszuschließen ist, dass diese Testkonzentration zu hoch gewählt ist.

\section{Bufexamac}

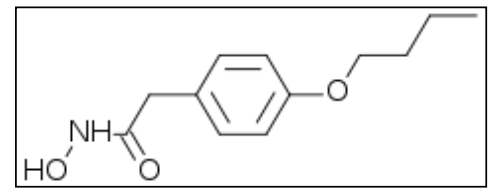

CAS Nr. 2438-72-4 (Korting 1995)

Bufexamac, eine nichtsteroidale antiphlogistische Substanz zur topischen Anwendung, wurde als Kortikoid-Ersatz zur Behandlungen von Ekzemen beworben und vor allem bei Kindern eingesetzt (Kränke et al. 1996). Es hat strukturelle Ähnlichkeiten zum Diclofenac und Naproxol (Perret und Happle 1989):<smiles>O=C(O)Cc1ccccc1Nc1c(Cl)cccc1Cl</smiles>

Diclofenac (Duggan et al. 2010)

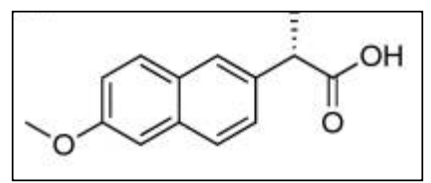

Naproxol (Duggan et al. 2010)

Zusätzlich ist Bufexamac in vielen Präparaten zur Behandlung von Perianalekzem und „Hämorrhoidalleiden“ enthalten (Schnuch et al. 2005a).

Schon Fuchs und Geier zeigten teils heftige kontaktallergische Reaktionen als Folge von Behandlungen von Patienten mit Molluscum contagiosum oder Pityriasis versicolor (Geier und Fuchs 1989). In unseren Göttinger Untersuchungen im Zeitraum zwischen 1996 und 1999 traten bei 2,1 \% ( $n=1536)$ positive Reaktionen auf. Unter anderem wegen dieser hohen Reaktionsquote wurde im Jahre 1999 Bufexamac in die DKG Standardreihe aufgenommen. Hier zeigte sich in der Folgezeit eine Sensibilisierungsquote von 1,4 \% (n=8531) (Schnuch et al. 2005a). In einer Studie aus Österreich wurde eine Quote von 5,7\% ( $n=141)$ publiziert (Kränke et al. 1996). Vor dem Hintergrund, dass diese Substanz zur Therapie von sehr unterschiedlichen Hauterkrankungen eingesetzt würde und sich häufige epidermale Sensibilisierungen zeigten, erscheint eine standardmäßige Testung sinnvoll. Im Anwendungsgebiet von Bufexamac gibt es wirksamere Medikamente mit weniger Nebenwirkungen, so dass in diesem Zusammenhang ein eindeutig negatives Nutzen-Risiko Verhältnis besteht (Schnuch et al. 2005a). In der Zwischenzeit wurde auf Grund dieses Risikoprofils Bufexamac seit Juni 2010 vom Markt in der EU genommen (European Medicines Agency 2010). 


\section{Cain-Mix 1}

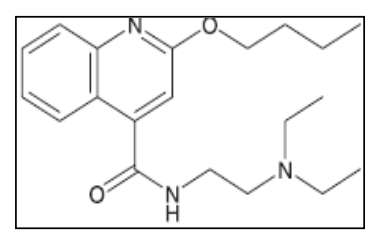

Dibucain (Cinchocain): CAS Nr. 85-79-0 (Le Coz et al. 2006) Procain: CAS Nr. 59-46-1 (Le Coz et al. 2006)

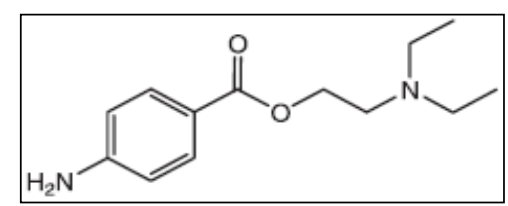

Der Cain-Mix besteht aus den zwei Bestandteilen Dibucain (Cinchocain) (1\%), einem Lokalanästhetikum vom Amid-Typ, und Procain (1\%), einem Lokalanästhetikum vom EsterTyp. Sie werden in Salben, Cremes, Augentropfen und als Sprühspray für den Rachen zur Linderung von Schmerzen eingesetzt. Der Cain Mix kam in Göttingen parallel zu dem in der Standardreihe getesteten Benzocain, ein Lokalanästhetikum vom Ester-Typ mit bekanntem Sensibilisierungspotential (Blaschke und Fuchs 2003), zum Einsatz. Dabei zeigten sich in 2,3\% ( $\mathrm{n}=611)$ positive Reaktionen. Die regelmäßige Cain-Mix Epikutantestung scheint demnach sinnvoll.

Daten aus europäischen Untersuchungen mit Benzocain zeigten relativ hohe Sensiblisierungsquoten mit 1,6\% $(n=26210)$ (Bruynzeel et al. 2005). Allerdings ist davon auszugehen, dass Benzocain allein nicht genügt, um eine allergische Kontaktdermatitis auf Lokalanästethika im Allgemeinen aufzudecken (Lodi et al. 1999). Schließlich wurde im Jahr 2005 Benzocain in die Lokalanästhetika Reihe platziert, in der u.a. auch Procain, Lidocain, Dibucain (Cinchocain) getestet werden. Außerhalb der Anwendung in entsprechenden medizinischen Präparaten hat es keine allgemeine Bedeutung als Allergen und daher reicht eine Anamnese-geleitete Testung aus. 


\section{Carba-Mix}
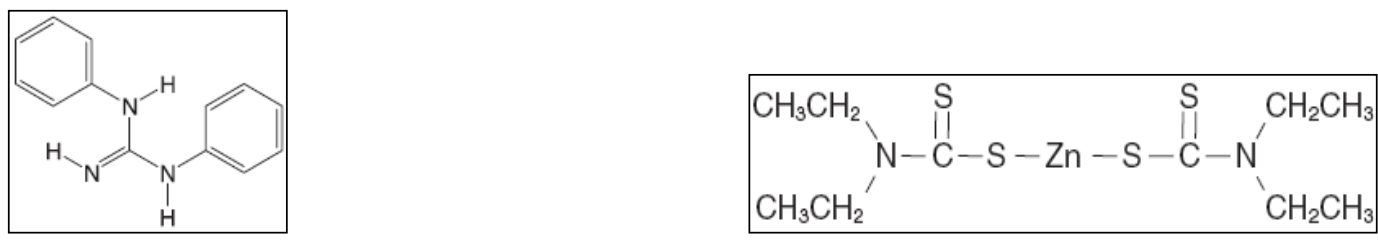

1,3- Diphenylguanidin: CAS Nr. 102-06-7

Zinkdiethyldithiocarbamat: CAS Nr. 14324-55-1

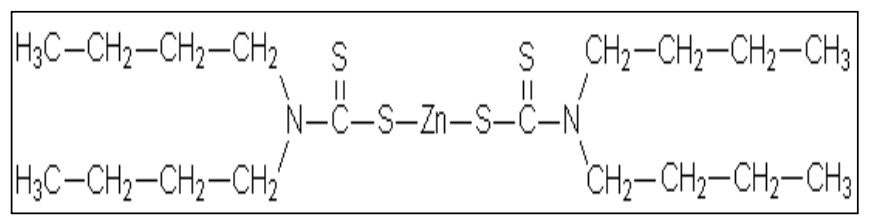

Zinkdibutyldithiocarbamat: CAS Nr. 136-23-2 (Le Coz et al. 2006) und (Geier et al. 2003c)

Der Carba-Mix wird verwendet, um bestimmte Formen von Gummiallergien aufzuklären und enthält die drei Bestandteile 1,3- Diphenylguanidin (DPG) 1\%, Zinkdiethyldithiocarbamat (ZDEC) 1\% und Zinkdibutyldithiocarbamat 1\% (ZDBC) (Sherertz und Swartz 1994). Alle drei Substanzen sind Vulkanisationsbeschleuniger von Gummiprodukten. Vulkanisation wird definiert als ein Verfahren und Vernetzungsprozess, bei dem der vorwiegend plastische Kautschuk durch Verknüpfung von Makromolekülen in den gummielastischen Zustand übergeht (Fuchs 1995) (siehe Zinkdiethyldithiocarbamat und Zinkdibutyldithiocarbamat Seiten 67 und 68). In den Göttinger Untersuchungen wurden in 3,1\% ( $n=388)$ positive Reaktionen beoabachtet. Dabei waren alle höchstens einfach positiv (+) bei einer hohen Anzahl an fraglichen Reaktionen. Dieses spiegelt sich im negativen RI (-0.6), einem Indiz für falsch positive Reaktionen (Geier et al. 2003d). Eine ähnliche Beobachtung wurde in einer Studie in den USA publiziert (Sherertz und Swartz 1994). In einer italienischen Studie wurde bei Medizinalpersonal eine Sensibilisierungsquote von 2,1\% $(n=360)$ beobachtet (Nettis et al. 2002). In nordamerikanischen Studien der NACDG wurden für den Carba Mix Raten von 7,3\% ( $\mathrm{n}=3437)$ (Marks et al. 2000) und 3,9\% ( $\mathrm{n}=4454)$ (Zug et al. 2009) gesehen. In einer Studie der Mayo-Clinic in den USA wurde eine Rate von 5,2\% publiziert (Wetter et al. 2005). Multizentrische Daten aus Europa berichten über Quoten von 2,68\% ( $n=12688)$ (Uter et al. 2016). Eine Erklärung für diese hohen Quoten findet sich wahrscheinlich in der Zusammensetzung vom Carba Mix wieder: Für die allergischen Reaktionen könnten hauptsächlich das ZDEC, ein bekanntes Allergen, welches in die Standardreihe der DKG aufgenommen ist (Schnuch et al. 2005c), verantwortlich gemacht werden. DPG, als ProblemAllergen bekannt (siehe Seite 38), kann ebenfalls allergische Reaktionen verursachen, hat jedoch ein hohes Potential an falsch positiven, d.h. irritativen, Reaktionen. Für ZDBC scheint 
ein geringes allergisches Potential vorzuliegen (siehe ZDBC Seite 68). Aus diesen Erkenntnissen lässt sich ableiten, dass eine routinemässige Testung vom Carba Mix nicht sinnvoll ist, da durch die Epikutantestung der einzelnen Substanzen eine differenziertere Aussage getroffen werden kann. Diese Meinung spiegelt sich aktuellen Diskussionen wieder (Uter et al. 2016).

\section{Carboxymethylcellulose}

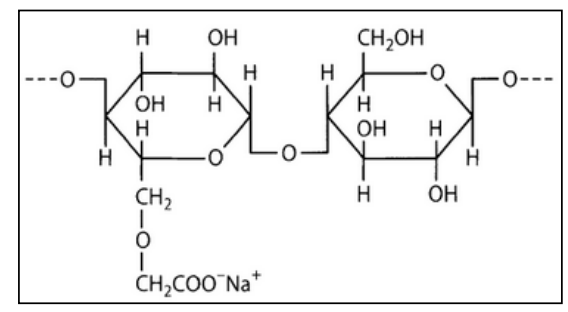

CAS Nr. 9004-32-4 (Thoma 2000)

Carboxymethylcellulose (CMC), auch als E 466 bekannt, ist ein Zusatz- und Bindemittel in der Lebensmittelindustrie (Hamada und Horiguchi 1978). CMC verhindert Verklumpungen und bindet Wasser z.B. in Süßwaren, Speiseeis oder Desserts. Chemisch ist es ein Abkömmling von Cellulose (E460), welches in der Nahrung ein häufig eingesetztes Zusatzmittel ist (Montoro et al. 2000). Medizinische Einsatzgebiete vom CMC sind hydrokolloidale Wundverbände (Koo et al. 2008) wie der Comfeel Verband, welcher z.B. bei der Behandlung von Ulcera im Rahmen einer chronisch venösen Insuffizienz, verwendet werden kann. CMC kann auch bei oberen und unteren Gastrointestinaluntersuchungen Bestandteil von Barium-Kontrastmittellösungen sein (Muroi et al. 2002). Auf Grund der breiten Verwendung und von Fallberichten über Sensibilisierungen auf Inhaltsstoffe von Verbänden bei Patienten mit Wundbehandlung (Koo et al. 2008) sowie einem Bericht über eine anaphylaktische Reaktion nach intra-artikulärer Injektion (Montoro et al. 2000) wurde in Göttingen CMC parallel zur Standardreihe getestet. Dabei zeigte sich keine $(0,0 \%)$ positive Reaktion ( $\mathrm{n}=2385)$. In der Literatur finden sich nur einzelne Fälle von Sensibilisierungen auf CMC (Barbaud et al. 2011). Insofern sollte eine Testung allenfalls gezielt Anamnese bezogen erfolgen. 


\section{Clobetasol-17-propionat}

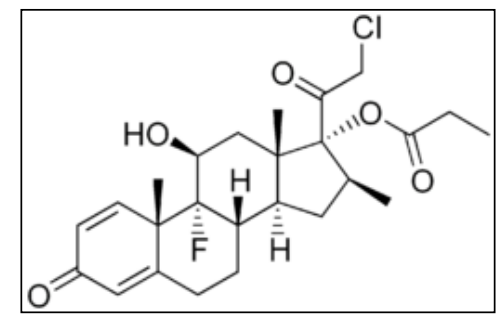

CAS Nr. 25122-46-7 (Gloor 2000)

Clobetasol-17-propionat (CP) (u.a. in Dermovate ${ }^{\circledR}$ ) ist ein stark wirksames Glukokortikosteroid der sog. Klasse D zur topischen Anwendung auf die Haut (Thomson, et al. 1999). Die Einteilung der topisch anwendbaren Glukokortikosteroide in verschiedenen Klassen richtet sich entweder nach ihrer Wirkstärke (Niedner 1996) oder nach der hier verwendeten Klassifikation, die chemische und allergologische Gesichtspunkte berücksichtigt. Demnach gibt es 4 allergene Haupt-Gruppen (A-D) (Matura und Goossens 2000), wobei das CP wie zum Beispiel Hydrocortison-17-butyrat und Prednicarbat in die Gruppe D eingeteilt wird, d.h. langkettiger Ester an C17 und/oder C21. Seit den 1950er Jahren bis heute werden topische Glukokortikosteroide gegen entzündliche und proliferative Hauterkrankungen eingesetzt und haben den Vorteil, dass sie eine relativ geringe systemische Wirkung und Toxizität haben (Isaksson 2004). Es besteht allerdings die Gefahr, dass eine Kontaktallergie durch Fehlinterpretation als Folge der vermeintlichen Krankheit maskiert wird (Gonul und Gul 2005). Da CP in Göttingen häufig zur Behandlung von Patienten mit Neurodermitis eingesetzt wurde, erfolgte eine Testung parallel zur Standardreihe, bei der in $0,1 \%$ ( $n=3219)$ positive Reaktionen beobachtet wurden. In fortlaufenden multizentrischen Untersuchungen des IVDK löste CP in 0,4\% ( $\mathrm{n}=5677)$ positive Reaktionen aus (Uter, et al. 2009). In einer multizentrischen britischen Studie wurde für CP eine Reaktionsquote von 0,8\% $(\mathrm{n}=1562)$ festgestellt (Sommer et al. 2002). Eine australische Untersuchung zeigte keine Reaktionen ( $n=82$ ) auf CP (Keegel et al. 2004). In einer der ersten Studien über Glukokortikoid Kontaktallergien wurde in Belgien eine Quote von 0,2\% ( $\mathrm{n}=2073)$ beobachtet (Dooms-Goossens und Morren 1992). In allen Untersuchungen war Vaseline als Vehikel im Einsatz. In einer nordamerikanischen Untersuchung mit CP (Petr.) wurde eine Rate von 0,3\% $(\mathrm{n}=17978)$ festgestellt (Pratt et al. 2017).

Eine routinemäßige Untersuchung ist nach diesen Ergebnissen nicht sinnvoll. Regelmäßig getestete Marker wie Tixocortol Pivalat und Budenosid könnten hier zur Untersuchung auf von Kontaktallergien auf Kortikosteroide ausreichen (Bruynzeel et al. 2005). 


\section{Dexpanthenol}

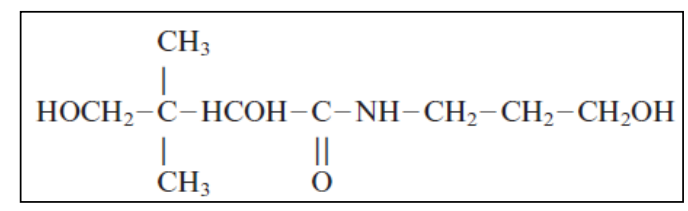

CAS Nr. 81-13-0 (Stables und Wilkinson 1998)

Dexpanthenol (u.a. in Bepanthen ${ }^{\circledR}$ ) wird im Körper zu Panthothensäure (Vitamin B5) umgewandelt. Panthothensäure ist ein Bestandteil des Coenzym A, das wiederum zu Aktivierung von Stoffwechselvorgängen führt (Stables und Wilkinson 1998). Diese Substanz wird in Externa, Kosmetika und darüber hinaus z.B. in systemisch zu verabreichenden Vitamin-Präparaten, eingesetzt (Skudlik et al. 2002). Die dermatologischen Wirkungen von Dexpanthenol sind unter anderem eine erhöhte Fibroblastenproliferation, eine beschleunigte Re-Epilthelisation in Wundgebieten und Hydratation im Stratum corneum der Haut, was vor eindringenden Irritantien schützt (Biro et al. 2003).

Anfang der 1980er Jahre gab es Berichte von teils sehr ausgeprägten Reaktionen auf Dexpanthenol (Ippen 1981). Um einen Eindruck von der Sensibilisierungsrate in Göttingen zu bekommen, wurde es fortlaufend und parallel zur Standardreihe getestet. Dabei wurden in 1,0\% ( $\mathrm{n}=2126)$ positive Reaktionen auf diesen Wirkstoff gesehen. In einer österreichischen Studie wurde Dexpanthenol ebenfalls parallel zur Standardreihe getestet und eine Sensibilisierungsquote von 0,34\% ( $\mathrm{n}=1474)$ beobachtet (Hemmer et al. 1997). Im Rahmen von multizentrischen Testungen des IVDK, in denen die Göttinger Zahlen vertreten waren, kam es in 1,2\% ( $n=13216)$ zu positiven Reaktionen (Skudlik et al. 2002). Die Autoren geben jedoch an, dass die Auswahl der getesteten Patienten zum großen Teil gezielt war, d.h. bei Patienten mit Unterschenkelekzem oder Gesichtsekzem. Dies sei der Grund der relativ hohen Quote. Finnische Untersuchungen zeigten Quoten von 0,7\% $(\mathrm{n}=286)$ und 0,5\% $(\mathrm{n}=5102)$ im Zeitraum von 1995 und 2002 (Hasan et al. 2005). Bei allen Tests wurde Vaseline als Vehikel verwendet. Eine Testung mit Dexpanthenol ist zurzeit nur bei anamestischem Verdacht sinnvoll. 


\section{Dibromdicyanobutan}

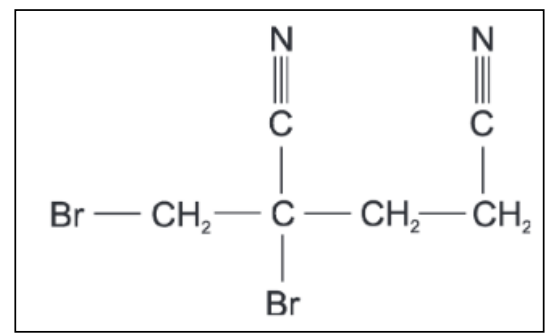

CAS Nr. 35691-65-7 (Dickel et al. 2015)

Dibromdicyanobutan (Methyldibromoglutaronitril (MDBGN) oder Tektamer 38) ist ein flüssiges Konservierungsmittel mit einem breiten Wirkungsspektrum gegen Pilze, Hefen und Bakterien (de Groot et al. 1996). Industriell wird MDBGN zur Konservierung von Klebstoffen, Polituren und Kühlschmierstoffen eingesetzt (Dickel et al. 2015). Seit Anfang der 80er Jahre fand MDBGN als ein Bestandteil vom Euxyl ${ }^{\circledR} \mathrm{K} 400$ (mit einem Anteil von ca. 20-25\%) Verwendung in Produkten wie z.B. Shampoos, Seifen, Badezusätzen oder Gesichtscremes und war damit eines der meist gebräuchlichen Konservierungsmittel für Kosmetika in Deutschland ist (Hausen 1993a). Die zweite Komponente des Euxyl ${ }^{\circledR}$ K 400 ist mit einem Anteil von ca. 80\% das Phenoxyethanol. Eine starke Zunahme des Einsatzes von Euxyl ${ }^{\circledR}$ K 400 führte zu einem Anstieg von Sensibilisierungen, weswegen es im Jahre 1991 in die Standardreihe der DKG aufgenommen wurde (Geier et al. 1996b). Während kontinuierlich eine Zunahme von Sensibilisierungen gegenüber MDGBN beobachtet wurde, waren Reaktionen gegenüber Phenoxyethanol Einzelfälle (Geier et al. 1996b). Um eine bessere Differenzierung dieser beiden Allergene vornehmen zu können, untersuchten wir MDGBN in Göttingen, im Rahmen einer multizentrischen Studie der DKG, in den Testkonzentrationen von $0,1 \%$ und $0,3 \%$ (jeweils in Vas.). Dabei zeigten sich in 2,1\% ( $\mathrm{n}=612)$ bzw. 4,9\% ( $\mathrm{n}=1102)$ positive Reaktionen. In einer IVDK-Studie wurde im Jahre 1996 publiziert, dass bei einer Testkonzentration von 0,1\% (Vas.) eine stetige Zunahme der Sensibilisierungsquote von 0,5\% auf 2,0\% im Zeitraum 1990 bis $1994 \mathrm{zu}$ verzeichnen war (Geier et al. 1996b). Für die gleiche Testkonzentration wurde in einer darauf folgenden großen deutschen multizentrischen Studie eine Quote von 1,8\% (n=11052) publiziert (Schnuch et al. 1998). Für die Testkonzentration von 0,3\% (Vas.) wurde in einer weiteren IVDK-Studie eine Quote von 3,3\% ( $\mathrm{n}=4615)$ gesehen (Geier et al. 2000b). Zuletzt schwankte die Rate bei Testungen des IVDK bei 2,1\% und 2,6\% (Dickel et al. 2015).

Der Trend mit zunehmend hohen Sensibilisierungsraten wurde in internationalen Untersuchungen bestätigt. Multizentrische finnische Studien zeigten einen Anstieg der Quoten von 1,0\% (n=5218) auf 1,5\% (n=11786) zwischen den Jahren 1995 und 2002 (Hasan et al. 2005). In einer britischen Studie wurde MDGBN mit einer Quote von 1,1\% ( $\mathrm{n}=6958)$ in 
0,3\% Konzentration (Vas.) getestet (De Jong et al. 2007). In Italien wurde eine Quote von 2,3\% bei einer Konzentration von 2,5\% (Vas.) ( $\mathrm{n}=919)$ beobachtet (Tosti et al. 1995). In nordamerikanischen Studien der NACDG liegen für MDBGN/PE kombinierte Sensibilisierungsquoten von 5,8\% vor (Zug et al. 2009). Aus Nordamerika wurde in der bislang einzigen Publikation, wo MDBGN in 0,5\% Konzentration (Vas.) getestet wurde, eine Quote von 0,27\% ( $\mathrm{n}=5167)$ gemeldet (Jacobs et al. 1995).

Für Phenoxyethanol (PE) (siehe Phenoxyethanol Seite 53) zeigten Studien deutlich geringere Quoten $(<0,5 \%)$. Somit ist MDBGN als das wesentliche Allergen anzusehen. In der Folge wurde 2005 als Konsequenz aus diesen Ergebnissen Euxyl ${ }^{\circledR}$ K 400 aus der Standardreihe der DKG gestrichen und durch MDGBN, das einzeln bis dahin im Kühlschmierstoff Block der DKG vertreten war, in 0,3\%ige Konzentration (Vas.) ersetzt (Geier und Mahler 2016). Zu gleicher Zeit erfolgte die Empfehlung zur Aufnahme von MDGBN in die europäische Standardreihe (Bruze et al. 2005). Allerdings blieb es noch unklar, welche Testkonzentration als die optimale angesehen werden kann. Einige Autoren empfehlen eine Testkonzentration von 0,2\% (Vas.) und nicht 0,3\% (Vas.) (Schnuch et al. 2005b).

\begin{tabular}{|l|l|l|l|l|}
\hline & $0,1 \%$ & $0,2 \%$ & $0,3 \%$ & $0,5 \%$ \\
\hline Göttingen 1996-2004 & $2,1 \%(\mathrm{n}=612)$ & & $4,9 \%(\mathrm{n}=1102)$ & \\
\hline Italien 1995 & & & & $2,3 \%(\mathrm{n}=919)$ \\
\hline USA 1995 & & & & $0,3 \%(\mathrm{n}=5167)$ \\
\hline IVDK 1990-1994 & $\begin{array}{l}0,5 \%(\mathrm{n}=1961) \\
2,0 \%(\mathrm{n}=4690)\end{array}$ & & & \\
\hline IVDK 1998 & $1,8 \%(\mathrm{n}=11052)$ & $3,3 \%(\mathrm{n}=4615)$ & \\
\hline IVDK 2000 & & & & \\
\hline Finnland 1995-2002 & $\begin{array}{l}1,0 \%(\mathrm{n}=5218) \\
1,5 \%(\mathrm{n}=11786)\end{array}$ & & $1,1 \%(\mathrm{n}=6958)$ & \\
\hline Grossbritannien 2007 & & & & \\
\hline Schweiz 2012 & & $1,7 \%(\mathrm{n}=1212)$ & & \\
\hline Niederlande 2012 & & $1,7 \%(\mathrm{n}=1212)$ & & \\
\hline Deutschland 2012 & & $2,1 \%(\mathrm{n}=573)$ & $3,2 \%(\mathrm{n}=2080)$ & \\
\hline Österreich 2012 & & & & $0 \%(\mathrm{n}=984)$ \\
\hline Italien 2012 & & & $0,3 \%(\mathrm{n}=1220)$ & \\
\hline Grossbritannien 2012 & & & $0,8 \%(\mathrm{n}=8909)$ & \\
\hline Litauen 2012 & & & $5,7 \%(\mathrm{n}=670)$ & \\
\hline Spanien 2012 & & & & $1,2 \%(\mathrm{n}=821)$ \\
\hline Dänemark 2012 & & & & $3,1 \%(\mathrm{n}=1318)$ \\
\hline
\end{tabular}

Tabelle 9: Zusammenfassung der MDBGN Daten (chronologische Reihenfolge)

Das hohe Sensibilisierungspotential führte im Jahre 2003 seitens der Europäischen Kommission zu einem Verbot von MDBGN aus Produkten, die nach der Anwendung nicht abgewaschen werden (sog. leave-on) und zu einer Limitierung des Einsatzes in abwaschbaren (sog. rinse-off-) Produkten (Schnuch et al. 2005b). Seit Februar 2011 darf MDBGN in der EU darüber hinaus nicht mehr in Beschichtungsschutzmitteln, Schutzmitteln für Fasern, Leder, Gummi und polymerisierten Materialien, Schutzmitteln für Baumaterialien sowie Schutzmitteln für Flüssigkeiten in Kühl- und Verfahrenssystemen eingesetzt werden (Dickel 
et al. 2015). Diese Maßnahmnen haben praktisch dazu geführt, dass eine MDGBN Exposition in der EU selten geworden ist (Dickel et al. 2015).

\section{Dimethylformamid}

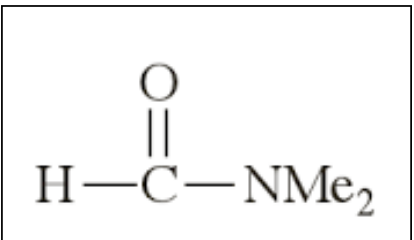

CAS Nr. 68-12-2 (Brückner 2015)

Dimethylformamid ist ein Lösungsmittel, das als Frostschutzmittel in Lacken, Farben, Harzen und in der Kunststoffindustrie eingesetzt wird (Cox und Mustchin 1991). Ende der 1990er Jahre wurde im Rahmen eines Fallbeispiels erstmals eine Sensibilisierung auf Dimethylformaid publiziert (Camarasa 1987). In Göttingen wurde daraufhin parallel zur Standardreihe die Sensibilisierungsquote untersucht. Dabei zeigten sich in 0,1\% $(n=1953)$ positive Reaktionen mit einem negativen RI (-0.8), einem Indiz für falsch positive, d.h. nicht allergische bzw. irritative, Reaktionen (Geier et al. 2003d). Eine Testung erscheint somit höchstens gezielt sinnvoll, wenn sich anamnestisch Hinweise auf einen entsprechenden Kontakt ergeben.

Nicht zu verwechseln ist Dimethylformamid mit Dimethylfumarat (DMF), welches als biozid zur Vermeidung von Schimmelbildung in Möbeln und Schuhe aber auch als Medikament (Fumaderm®) zur Behandlung von Psoriasis sowie der Multiple Sklerose eingesetzt wird (Lammintausta et. al 2010). Ab dem Jahre 2007 wurden in mehreren EU Ländern teils heftige allergische Reaktionen auf DMF, sog. „Sofa Dermatitis“, nach Exposition mit aus China importierten Möbeln festgestellt, woraufhin die Verwendung von DMF in Konsumgütern in der EU ab 2009 verboten wurde (Susitaival et al. 2010).

\section{1,3-Diphenylguanidin}<smiles>N=C(Nc1ccccc1)Nc1ccccc1</smiles>

CAS Nr. 102-06-7 (Le Coz et al. 2006)

Diphenylguanidin (DPG) ist ein Vulkanisationsbeschleuniger für Gummiprodukte und findet Verwendung in Reifen, Gummistiefeln (Fuchs 2007b) und seltener in Handschuhen (Geier et 
al. 2003a). Zuletzt war ein Trend zu beobachten, dass zunehmend Handschuhe aus natürlichem Gummi (Latex) durch Handschuhe aus synthetischem Gummi ersetzt werden, in denen DPG vermehrt vorkommen kann (Piskin et al. 2006). Unsere Epikutantestungen mit DPG in einer Konzentration von 1\% (Vas.) führten in 1,6\% $(n=385)$ zu positiven Reaktionen. Auffallend war, dass eine überwiegende Anzahl an Reaktionen schwach positiv war und gleichzeitig eine relativ hohe Anzahl an fraglichen Reaktionen vorlag. Dies spiegelt sich im negativen RI (-0,7), einem Indiz für falsch positive Reaktionen, wider (Geier et al. 2003d). Diese Beobachtung konnte im Rahmen einer multizentrischen Studie des IVDK, in der die Göttinger Daten vertreten waren, bei einer Sensibilisierungsquote von 1,9\% ( $n=1455)$ bestätigt werden (Geier et al. 2003a). Bei einer späteren IVDK Studie bei Patienten mit berufbedingter Dermatose fand sich eine Quote 3,0\% ( $n=3448)$ (Geier et al. 2012). In einer spanischen Studie zeigte DPG eine Quote von 2,3\% ( $\mathrm{n}=686)$ (Conde-Salazar et al. 1993). Aktuelle multizentrische europäische Daten berichten von Quoten von 3,26\% (Uter et al. 2016). In allen o.g. Studien wurde DPG in einer Konzentration von 1\% (Vas.) getestet. In der Literatur gibt es Hinweise, dass die hohe Anzahl an fraglich positiven Reaktionen möglicherweise auf eine $\mathrm{zu}$ hohe Testkonzentration zurückgehen könnte und evtl. eine Testkonzentration von 0,1\% geeigneter wäre (Fuchs 1995).

Es lässt sich schlussfolgern, dass eine Testung zumindestens bei Verdachtsmomenten auf eine Gummiallergie, z. B. im Rahmen der Gummireihe der DKG, in der DPG vertreten ist, erfolgen sollte. Es gibt sogar Diskussionen, DPG in die Standardreihe aufzunehmen (Uter et al. 2016). Eine Testung im Rahmen des Carba-Mixes erscheint somit nicht sinnvoll, da durch Epikutantestungen der einzelnen Substanzen eine differenziertere Aussage getroffen werden kann (siehe Carba-Mix Seite 32).

\section{Dispersions Blau 124 und Mix 124/106}

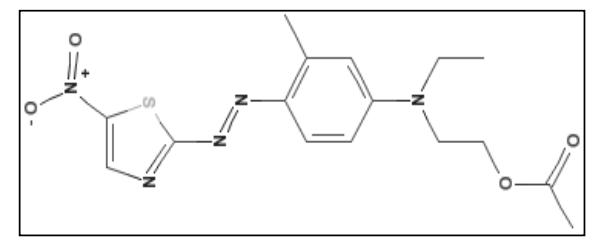

Dispersions Blau 124: CAS Nr. 61951-51-7

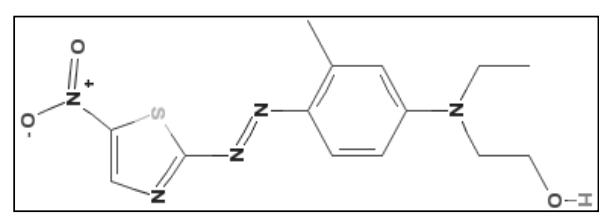

Dispersions Blau 106: CAS Nr. 12223-01-7

(Le Coz et al. 2006)

Dispersions Blau (DB) 124 und der DB-Mix, bestehend aus den beiden Komponenten DB 106 und DB 124, sind Textilfarbstoffe, die unter anderem zur Färbung von Unterwäsche, Hosen und Oberbekleidung verwendet werden (Maurer et al. 1995). Seit den 1980er Jahren gibt es Hinweise, dass DB 106 und 124 ein nicht unerhebliches Sensibilisierungspotential 
haben (Hausen 1993b). Im Rahmen von multizentrischen Studien des IVDK testeten wir DB Mix 124/106 und DB 124 parallel zur Standardreihe. Dabei zeigten sich für DB Mix 124/106 in $1,6 \%(n=62)$ positive Reaktionen und für DB 124 in $0,7 \%(n=612)$ positive Reaktionen (jeweils $1 \%$ in Vas.). Die Gesamtpopulation des IVDK zeigte für DB Mix 124/106 in 1,1\% $(\mathrm{n}=2415)$ positive Reaktionen (Aberer et al. 2003). Chemische Analysen zeigten, dass DB 124 nur durch einen Acetylrest von DB 106 zu unterscheiden ist und zudem so instabil ist, dass DB 106 stets mit DB 124 kontaminiert sein dürfte (Aberer et al. 2003). Im Jahre 2002 erschien die Verwendung von DB Mix 124/106 und die Aufnahme in die Standardreihe sinnvoll, jedoch ergaben in den folgenden Jahren u.a. auch durch unsere Untersuchungen, dass es in nicht mehr als 0,6\% $(\mathrm{n}=24980)$ zu positiven Reaktionen kam (Uter et al. 2007b), so dass im Jahre 2006 der DB Mix 124/106 ersatzlos aus der Standardreihe gestrichen wurde, zumal dieser Mix vom Hersteller nicht mehr angeboten wurde. Seitdem gilt die Empfehlung, DB 106 und DB 124 als Einzelkomponenten in der Testkonzentration von 0,3\% (Vas.), in dem so genannten Leder und Textilfarben Block der DKG zu testen (Geier und Mahler 2016). Aktuelle Daten des IVDK zeigen bei Testungen von Patienten mit Verdacht auf Textilallergie Reaktionsquoten von 2,3\% $(n=3207)$ bei DB 124 und 2,0\% $(n=3207)$ bei DB 106 (Heratizadeh et al. 2017). Bei einer Konzentration von $1,0 \%$ wurden iatrogene Sensibilisierungen durch den Test vermutet (Kimber et al. 2005). Die o.g. Substanzen sollen mittlerweile in Deutschland nicht mehr zur Textilfärbung verwendet werden, da sie zu den potentiell stärksten sensibilisierenden Allergenen in Bekleidung gehören (Frosch et al. 2006), wobei eine Expositionsmöglichkeit über Importware aber nach wie vor gegeben ist.

\section{Glutaraldehyd}

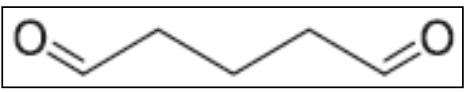

CAS Nr. 111-30-8 (Le Coz et al. 2006)

Glutaraldehyd (GA) ist ein häufig verwendetes Kalt-Sterilisationsmittel mit bakteriziden und viroziden Eigenschaften (Shaffer und Belsito 2000) und wird als Desinfektionsmittel von medizinischem Gerät, z.B. in Zahnarztpraxen und operativen Bereichen, aber auch von Friseurutensilien eingesetzt (Kiec-Swierczynska und Krcisz 2001). Vor allem Berufsgruppen wie Krankenschwestern, Technische Assistenten, Reinigungskräfte und Friseure können gehäuft exponiert sein (Geier und Schnuch 1995). In den letzten Dekaden, im Zeitalter viral übertragbarer Krankheiten (z.B. HIV, Hepatitis), hat der Gebrauch von Glutaraldehyd trotz bekannter irritativer Wirkung auf die Haut (Hansen und Menne 1990) zunehmende Beliebtheit erlangt. Gleichzeitig wurden zunehmend Dermatitiden und Reizungen des 
Respirationstraktes beschrieben, die in Zusammenhang mit allergischen Reaktionen gebracht wurden (Shaffer und Belsito 2000). Daher erfolgten in Göttingen für GA in einer Konzentration von 0,3\% (Vas.) Testungen parallel zur Standardreihe, in denen bei 0,3\% ( $n=3218)$ positive Reaktionen beobachtet wurden. Die Anzahl irritativer Reaktionen war um den fünffachen Faktor erhöht. Dies spiegelt sich im negativen RI (-0,8), einem Indiz für falsch positive Reaktionen wieder (Geier et al. 2003d). In nordamerikanischen Studien wurden in einer Studie der Mayo-Clinic eine Quote von 5,5\% $(n=1040)$ (Wetter et al. 2005) und in zwei Studien der NACDG Quoten von 1,9\% ( $n=5802)$ (Marks et al. 2003) und 2,6\% $(n=4094)$ (Marks et al. 2000) gesehen. Die Ursache für die deutlich höheren Sensibilisierungsquoten als bei uns könnte bei den genannten Studien in einer höheren Testkonzentration (1\% in Vas.) liegen und sind nach den Ergebnissen der eigenen Untersuchungen sehr wahrscheinlich irritativer Natur. Für eine Testkonzentration von 0,2\% (Vas.) wurde in Nordamerika eine Quote von 1,7\% ( $\mathrm{n}=4096)$ publiziert (Marks et al. 2000). Da Glutaraldehyd fast nur für Berufstätige im Krankenhaus und Raumpfleger relevant ist, ist eine regelmäßige Testung mittels Standardreihe nicht $\mathrm{zu}$ empfehlen. Testungen somit sollten gezielt nach anamnestischen Angaben erfolgen.

\section{Hexylzimtaldehyd}

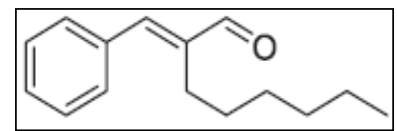

CAS Nr. 101-86-0 (Le Coz et al. 2006)

Hexylzimtaldehyd, auch unter dem englischen Namen alpha-hexyl-cinnamic-aldehyde (AHCA) bekannt, ist ein Duftstoff, der u. a. in Kosmetika, Parfums und Deodorants eingesetzt wird (Frosch et al. 2002). Duftstoffe wurden üblicherweise mit einer Mischung, dem Duftstoff-Mix I, der acht Inhaltsstoffe (Zimtalkoholaldehyd, alpha-Amylcinnamylaldehyd, Eugenol, Isoeugenol, Hydroxycitronellal, Geraniol und Eichenmoos) beinhaltet, in der Standardreihe regelmäßig getestet. Es zeigte sich jedoch, dass nicht alle Duftstoffe, die zu Sensibilisierungen führen können, mittels dieser Duftstoff-Mischung entdeckt wurden (de Groot, et al. 1993). So erfolgte in Göttingen parallel zur Standardreihe die Testung auf weiterer Duftstoffe wie AHCA (2\% in Vas.). Dabei zeigten sich keine positive Reaktionen $(n=792)$. In einer multizentrischen Studie des IVDK zeigte sich eine Sensibilisierungsquote von $0,1 \%(n=2019)$ bei einer Konzentration von 10\% (Vas.) (Schnuch et al. 2007b). Eine multizentrische europäische Studie zeigte eine Quote von 0,3\% (10\% in Vas.) $(n=1606)$ (Frosch et al. 2002). Somit scheint AHCA kein häufiges Allergen zu sein. Für den strukturverwandten, jedoch nicht identischen, Zimtaldehyd (engl. cinnamic aldehyde) liegen 
deutlich höhere Quoten 1,7\% ( $\mathrm{n}=1321)$ (Wetter et al. 2005) und 3,6\% ( $\mathrm{n}=5831)$ (Marks et al. 2003) im nordamerikanischen Studien vor. Mittlerweile kommen zwei Duftstoff-Mixe in der Standardreihe regelmäßig zum Einsatz, da gezeigt werden konnte, dass dadurch zusätzliche Sensibilisierungen entdeckt werden (Frosch et al. 2005). AHCA ist ein Bestandteil des Duftstoff-Mix II (Hexylzimtaldehyd, Citral, Citronellol, Cumarin, Farnesol und Lyral). Daher sollte die regelmäßige Testung in diesem Mix auf Grund der oben genannten Ergebnisse diskutiert werden.

\section{Hg.-Präcipitatsalbe}

$\left[\mathrm{Hg}\left(\mathrm{NH}_{2}\right)\right]^{+} \mathrm{Cl}$

Quecksilber (II)-amidchlorid : CAS NR. 10124-48-8

Lanolin: CAS Nr. 8006-54-0

(Koch 2007)

Präcipitatsalbe (Hg.= Quecksilber) setzt sich aus Quecksilber (II)-amidchlorid (ca. 10\%), Lanolin (Wollwachs), Vaseline, Ammoniaklösung und Wasser zusammen (Winter 1932) und wurde zur topischen Lokaltherapie bei entzündlichen Erkrankungen eingesetzt (Steigleder 1987). Wollwachs, ein Sekret aus Talgdrüsen von Schafen, besitzt neben hautpflegenden Eigenschaften auch ein gewisses Sensibilisierungspotential (Steigleder 1987). Seit langem gibt es bezüglich Intoxikationen und Sensibilisierungen auf quecksilberhaltige Stoffe kontroverse Diskussionen (Fuchs 1994). In Göttingen wurden in den 1990er Jahren parallel zur Standardreihe quecksilberhaltige Stoffe untersucht, unter anderem im Hinblick auf Sensibilisierungsquoten und optimale Testkonzentrationen. In unseren Untersuchungen wurde bei Hg.-Präcipitatsalbe (Konzentration $1 \%$ in Vas.) in 4,2\% positive Reaktionen $(n=213)$ beobachtet. Eine frühere Göttinger Arbeit zeigte für die gleiche Konzentration (1\% Vas.) eine Quote von 3,0\% (n=3240) (Mintrop 1994). Eine multizentrische Studie der DKG, bei der verschiedene quecksilberhaltige Substanzen bei Patienten mit Verdacht auf Quecksilberallergie verglichen wurden, konnte zeigen, dass auf Grund einer guten Korrelation Quecksilber (II)-amidchlorid als Marker ausreichend ist, um Sensibilisierungen gegenüber quecksilberhaltige Stoffe im Allgemeinen aufzudecken (Mayenburg von et al. 1996). Quecksilber (II)-amidchlorid wurde darufhin als Marker in der Standardreihe aufgenommen. Da Reaktionen, insbesondere unerwartete, ohne zusätzliche Testungen oft kaum zu interpretieren waren, und als Quelle der Quecksilberexposition am häufigsten Dentalwerkstoffe verdächtigt wurden, wurde diese Testsubstanz im Jahre 2005 aus der Standardreihe gestrichen und in die Testreihe "DKG Dentalmetalle" (Block Nr. 17) verlagert 
(Geier und Mahler 2017). Hier wird es seitdem parallel zur Standardreihe getestet wird, falls indiziert.

\section{Hydrocortison-17-butyrat}

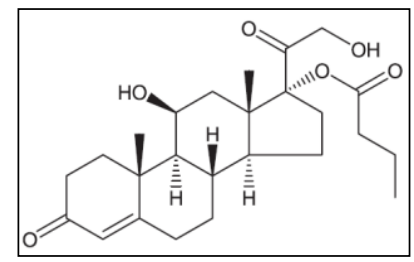

CAS Nr. 13609-67-1 (Le Coz et al. 2006)

Hydrocortison-17-butyrat (HB) ist ein mittelstark wirksames topisches Glukokortikosteroid (Handelsname z.B. Alfason ${ }^{\circledR}$ ) der sog. Klasse D zur topischen Anwendung auf die Haut (Matura und Goossens 2000). Die Einteilung der topisch anwendbaren Glukokortikosteroide in Klassen richtet sich entweder nach ihrer Wirkstärke (Niedner 1996) oder nach der hier verwendeten Klassifikation, die chemische und allergologische Gesichtspunkte berücksichtigt. Demnach gibt es 4 allergene Haupt-Gruppen (A-D) (Matura und Goossens 2000), wobei das HB wie zum Beispiel Clobetasol-17-propionat und Prednicarbat in die Gruppe D eingeteilt wird, d.h. langkettiger Ester an C17 und/oder C21.

Seit den 1950er Jahren bis heute werden topische Glukokortikosteroide gegen entzündliche und proliferative Hauterkrankungen eingesetzt (Isaksson 2004). Bei topischen Glukokortikoiden besteht die Möglichkeit, dass eine Kontaktallergie durch die Fehlinterpretation der vermeintlichen Krankheit maskiert wird (Gonul und Gul 2005). Da HB in Göttingen unter anderem häufig zur Behandlung von Neurodermitis eingesetzt wurde, erfolgte bei uns die Epikutantestung parallel zur Standardreihe, bei der wir in 0,2\% ( $\mathrm{n}=944)$ positive Reaktionen bei einer Konzentration von $0,1 \%$ (Vas.) beobachteten. In einer multizentrischen Studie des IVDK konnte für HB (0,1\% in Vas.) eine Sensibilisierungsquote von 1,5\% (n=5817) zwischen 1995 bis 2004 beobachtet werden (Uter et al. 2009). In einer australischen Studie wurden keine Reaktionen beschrieben (1\% in Vas.) (n=82) (Keegel et al. 2004). In einer der ersten Studien zu Kortikoidallergien wurde aus Belgien eine Quote von $1,0 \%$ (1\% in Eth.) ( $\mathrm{n}=2073)$ publiziert (Dooms-Goossens und Morren 1992). Eine regelmäßige Testung scheint nicht sinnvoll, jedoch zeigt vor allem das IVDK-Ergebnis, dass ein Sensibilisierungspotential besteht. HB sollte zumindest gezielt anamnestisch oder mit dem sog. Kortikoidblock getestet werden.

Zur Erfassung von Glukokortikosteroid-Allergien werden Markersubstanzen eingesetzt und für HB wurde Budesonid als geeignet beschrieben (Isaksson 2004). 


\section{Imidazolidinylharnstoff (Germall 115)}

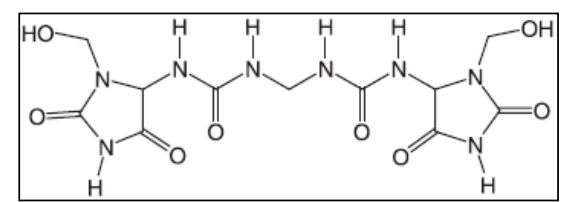

CAS Nr. 39236-46-9 (Le Coz et al. 2006)

Imidazolidinylharnstoff (IMID) (Handelsname Germall ${ }^{\circledR} 115$ ) hat eine breite antimikrobielle Wirkung und wird als Konservierungsstoff häufig in Kosmetika, Shampoos, Deodorants, Medikamenten und Salben eingesetzt (Fisher 1975).

Auf Grund seiner Strukturformel gehört IMID zur Gruppe der sog. Formaldehydabspalter, d.h. Konservierungsmittel, die in Anwesenheit von Wasser Formaldehyd freisetzen (Brasch und de Groot 2007). Es besitzt somit irritative Eigenschaften, jedoch setzt es relativ gesehen in dieser Gruppe die geringste Menge Formaldehyd frei, während z.B. Quaternium-15 die grösste Menge freisetzt (Brasch und de Groot 2007). Gleichzeitig weist IMID auch ein eigenes sensibilisierendes Potential auf (Lehmann et al. 2006).

Seit der Markteinführung von IMID in den 1970er Jahren wurden immer wieder Fälle von allergischen Kontaktdermatitiden beschrieben (Agner et al. 2001). Im Rahmen einer multizentrischen Studie testeten wir IMID in einer Konzentration von 1\% (Vas.). Dabei zeigten sich in $0,5 \%(\mathrm{n}=3214)$ positive Reaktionen. Nordamerikanische Studien zeigten Sensibilisierungsquoten von $2,7 \%$ bzw. 4,3\% $(\mathrm{n}=3028)$ bei Testungen mit Konzentrationen von $1 \%$ und 2\% (jeweils in Aqua) (Warshaw et al. 2009) und Quoten von 3,2\% (2\% Vas, $\mathrm{n}=4094$ ) sowie 2,5\% (2\% Aqua, $\mathrm{n}=4101$ ) (Marks, et al. 2000). Britische Studien zeigten bei Testungen mit einer Konzentration von 2\% (Vas.) Quoten von 1,0\% (n=5167) (Jacobs, et al. 1995) und 0,9\% (n=6958) (Jong et al. 2007). Eine türkische Studie ergab eine Quote von 0,3\% $(\mathrm{n}=308)$ (Boyvat et al. 2005) und eine schweizer Studie 1,0\% ( $\mathrm{n}=2295)$ (Perrenoud et al. 1994) mit jeweils 2\%iger (Vas.) Konzentration. Multizentrische finnische Studien zeigten Quoten von $1,1 \%(\mathrm{n}=1954)$ und $0,8 \%(\mathrm{n}=11794)$ bei einer Konzentration von $2 \%$ (Aqua) im Zeitraum von 1995 bis 2002 (Hasan et al. 2005). Bezüglich des Vehikels wurde berichtet, dass eine Testung von IMID in Vaseline eine signifikant sensitivere Detektionsrate von Sensibilisierungen gegenüber wäßrigen Lösungen (Aqua) erzielt (Rietschel et al. 2007).

Auf Grund der genannten Ergebnisse ist eine Aufnahme von IMID in die Standardreihe nicht sinnvoll. Es ist Bestandteil des Konservierungsmittel Blocks der DKG. Gegebenfalls sind Testungen gezielt nach anamnestischen Angaben und bei Hinweisen auf eine Kosmetikallergie sinnvoll. 
Incidin ${ }^{\circledR}$ Plus

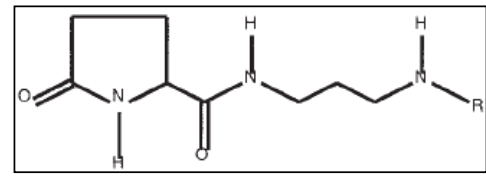

Glucoprotamin: CAS Nr. 164907-72-6 (Widmer und Frei 2003)

Incidin ${ }^{\circledR}$ Plus, ein flüssiges Konzentrat, wird u. a. in Krankenhäusern zur Flächendesinfektion wegen seiner Wirksamkeit gegen Bakterien, Pilzen und auch begrenzt gegen Viren eingesetzt (Widmer und Frei 2003) und gilt als biologisch schnell abbaubar (Ecolab 2017). Der Hauptwirkstoff (ca. 30\% Bestandteil) ist Glucoprotamin (Ecolab 2017), welcher seit Mitte der Neunziger Jahre auf dem Markt erhältlich ist und ein breites Einsatzspektrum hat (Disch 1994). Glucoprotamin $®$ entsteht als Reaktionsprodukt bei der Umsetzung von Kokospropylen-1,3-diamin mit L-Glutaminsäure (Steber und Schröder 1996). Das Incidin folgte einem Trend der letzten Dekaden, wonach die Zahl der Flächendesinfektionsreiniger von ursprünglich 3 auf 175 angestiegen waren (Wallhäuser 1988). Zur Erhebung eines Sensibilisierungspotentials zeigten unsere Epikutantests mit Incidin Plus in 0,1\% (Vas.) Konzentration keine $(0,0 \%)$ positive Reaktion $(n=263)$. In der Literatur ist, soweit recherchierbar, bisher kein Fall einer allergischen Reaktion gegenüber Incidin Plus und Glucoprotamin publiziert worden. Incidin Plus ist zur Zeit kein relevantes Kontaktallergen.

\section{Iodpropinylbutylcarbamat}

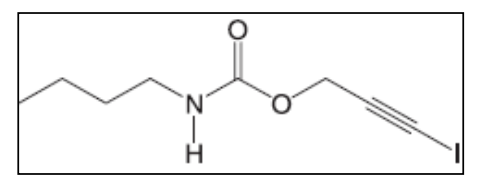

CAS Nr. 55406-53-6 (Le Coz et al. 2006)

Iodpropinylbutylcarbamat (IPBC) ist ein Konservierungsmittel mit bioziden Eigenschaften und wird insbesondere in der holzverarbeitenden Industrie verwendet (Schnuch et al. 2002a). Die EU hat IPBC im Jahre 1998 zur Verwendung in Kosmetika in einer Konzentration von 0,1\% zugelassen (Natkunarajah et al. 2008). Seither wird es zunehmend in Shampoos, Make Up, Cremes und Babyprodukten eingesetzt. IPBC stand im Verdacht, sensibilisierend zu sein und es häuften sich Fallberichte über allergische Reaktionen (Natkunarajah et al. 2008). In Göttingen testeten wir IPBC parallel zur Standardreihe. Hierbei zeigten sich für zwei Testkonzentrationen $(0,1 \%$ und $0,5 \%$, jeweils in Vas.) keine positiven Reaktionen $(n=44)$. In einer NACDG-Untersuchung aus Nordamerika wurde eine Quote von 0,4\% ( $n=5770)$ bei einer Konzentration von 0,1\% (Vas.) publiziert (Marks et al. 2000). Eine multizentrische Studie des IVDK zeigte eine Sensibilisierungsquote von $0,3 \%(n=4883)$, wobei die Autoren 
anmerkten, dass auf Grund der niedrigen Testkonzentration (0,1\% in Vas.) falsch negative Reaktionen anzunehmen seien (Schnuch et al. 2002a). Um dies zu prüfen und eine optimale Testkonzentration zu definieren, wurde IPBC in einer weiteren multizentrischen Studie des IVDK in verschiedenen Konzentrationen getestet. Hierbei zeigten sich abhängig von der Konzentration $(0,1 \%, 0,2 \%, 0,3 \%, 0,5 \%$, alle in Vas.), ansteigende Sensibilisierungsraten $(0,5 \%, 0,8 \%, 1,3 \%, 1,7 \%)(n=8106)$. Gleichzeitig stieg auch überproportional die Anzahl irritatitver Reaktionen (Brasch et al. 2004). Schließlich kamen die Autoren zu dem Schluss, dass eine Testkonzentration von $0,2 \%$ (Vas.) geeignet für weitere Untersuchungen erscheint (Brasch et al. 2004). Akutelle Daten aus Europa mit dieser Konzentration zeigen Raten zwischen $0 \%(n=624)$ in Litauen sowie Polen und 1,7\% $(n=1337)$ in den Niederlanden (Uter et al. 2012).

Unsere Ergebnisse ergeben keinen Hinweis darauf, dass IPBC ein starkes Allergen ist und allenfalls gezielt bzw. bei anamnestischen Hinweisen getestet werden sollte.

\section{$\mathrm{KKM}^{\circledR} 702$}<smiles>CC(=O)C1C(O)CC(C)OC1O</smiles>

Dehydroacetatsäure CAS Nr. 520-45-6

(Schülke 2006)

Polyaminopropyl biguanid CAS Nr. 27083-27-8 (Schülke 2006)

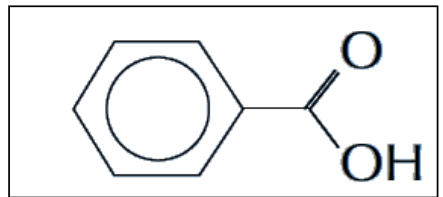

Benzoesäure CAS Nr. 65-85-0 (Schülke 2006)

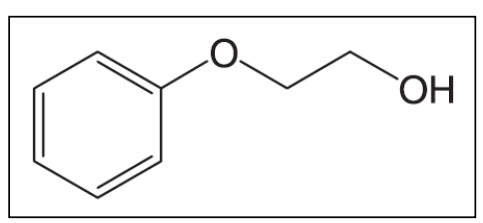

Phenoxyethanol CAS Nr.122-99-6

(Le Coz et al. 2006)
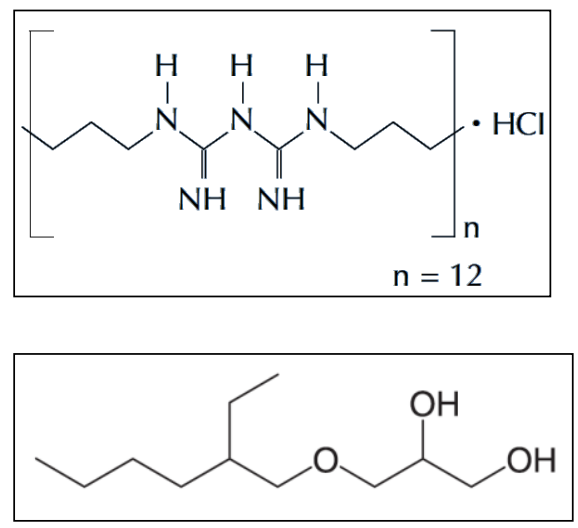

Ethylhexylglycerin: CAS Nr. 70445-33-9 (Le Coz et al. 2006)

$\mathrm{KKM}^{\oplus} 702$ (Synonym Euxyl $^{\circledR} \mathrm{K}$ 702) ist ein Gemisch auf der Basis von Benzoesäure, Dehydroacetatsäure, Phenoxyethanol, Polyaminopropyl biguanid und Ethylhexylglycerin. Es wird laut Firmenbroschüre als flüßiges Konservierungsmittel in kosmetischen Produkten wie 
Cremes, Lotionen oder Shampoos eingesetzt (Schülke 2006). Es ist ein biologisch wirksames Produkt mit biostatischer und biozider Wirkung gegenüber Hefen, Bakterien und Schimmelpilzen und ist vor allem in pH Bereichen <6 wirksam (Schülke 2006).

In unseren Tests konnte bei $\mathrm{KKM}^{\oplus} 702$ (1\% Vas.) keine positive Reaktion gesehen werden $(\mathrm{n}=2379)$. Bislang liegen unseren Wissens keine Publikationen zu Epikutantests oder weiteren Fallberichte vor. Phenoxyethanol ist als Bestandteil des $\mathrm{KKM}^{\circledR} 702$ als ein gering sensibilisierendes Allergen bekannt (siehe Phenoxyethanol Seite 54). Eine regelmäßige Testung vom $\mathrm{KKM}^{\circledast} 702$ erscheint trotz der breiten Verwendung auf Grund der oben genannten Ergebnisse derzeit nicht sinnvoll.

\section{Kolophonium-Mix II}

Kolophonium, ein bräunliches Naturharz und gewonnen aus Kiefern, Tannen und Fichten (Hausen et al. 1982a), wird in einer Vielzahl von verschiedenen Produkten, wie Papier, Kartons, Pflaster, Farben, Lacke, Klebstoffe, Seifen und Kaugummi eingesetzt (Hausen et al. 1982b). Natives Kolophonium, bekannt als eines der allergologisch bedeutendsten Substanzen mit Sensibilisierungsquoten um 4,5\% (Geier und Hausen 2006), kann chemisch modifiziert werden, um beispielsweise farblose Eigenschaften zu erlangen. Es ist möglich durch diesen Vorgang die sensibilisierenden Eigenschaften jedoch zu verstärken (Hausen und Mohnert 1989). Um die Situation zu überprüfen wurde Ende der 1990er Jahre in einer multizentrischen Studie des IVDK parallel zum nativen Kolophonium, das bereits in der Standardreihe der DKG war, auch ein Kolophonium-Mix I, bestehend aus gleichen Teilen chinesisches Kolophonium, Kalkzinkhartharz und ein Maleinsäurederivat (Hersteller: Prof. Hausen, Buxtehude), getestet. Dabei zeigte sich, dass 11,5\% mehr Allergiker durch die zusätzliche Testung zum nativem Kolophonium entdeckt wurden (Geier und Hausen 2000). In einer Nachfolgeuntersuchung an der sich Göttingen beteiligte, wurde im Rahmen einer weiteren multizentrischen Studie des IVDK Anfang 2000 eine Testung mit einem Kolophonium Mix II durchgeführt. Es hatte eine modifizierte Zusammenstellung, die sich an den auf dem Markt befindlichen kolophoniumhaltigen Produkten, nämlich einen Glycerinester, ein Tallharz und einen Pentaerythritester orientierte (Aberer et al. 2003). Dabei betrug die Göttinger Quote bei einer Konzentration von $15 \%$ (Vas.) $1,7 \%(n=179)$. Die Sensibilisierungsquote des IVDK entsprach ebenfalls 1,7\% (15\% in Vas.) ( $\mathrm{n}=3787)$ (Geier und Hausen 2006). Diese Studie zeigte zudem, dass in 8,3\% Kolophonium-Allergiker diagnostiziert werden konnten, die bei alleiniger Testung mit dem nativen Kolophonium unentdeckt geblieben wären (Geier und Hausen 2006). Die genannten Ergebnisse lassen eine Ergänzung der Testung in der 
Standardreihe mit dem Kolophonium-Mix II zum nativen Kolophonium als diagnostisch sinnvolle Maßnahme erscheinen.

\section{Latexhandschuhextrakt (4\% ige Pricklösung)}

Naturgummilatex (syn. Latex) ist eine kolloidale Dispersion von Kautschuk in Wasser, wobei zwischen Natur- und Synthesekautschuk unterschieden wird, je nach Kautschukart (Fuchs 1995). Naturlatex kann aus dem kautschukartigen Milchsaft von verschiedenen Pflanzenarten gewonnen werden. Wirtschaftlich am bedeutsamsten ist Hevea brasiliensis, deren Hauptanbaugebiet sich in Malaysia und Indonesien befindet (Fuchs 1995). Direkt nach dem Zapfen besteht es aus einem 50-60\% wäßrigen Anteil, aus einer 30-40\% dispersen Kautschukphase und zu 5-8\% aus Nichtkautschukbestandteilen. Durch Trocknung entsteht Latex Gummi, dessen rasche bakterielle Zersetzung u.a. durch Zugabe von Ammoniak als Stabilisator verhindert werden kann (Fuchs 1995). Industrielle Verwendung im medizinischen Bereich findet Latex vornehmlich als Schutz- und Operationshandschuh. Für Latex Gummi sind allergische Sofortreaktionen bekannt (Wakelin und White 1999). Um Hinweisen bezüglich eines allergischen Kontaktekzems nachzugehen, führten wir parallel zur Standardreihe Epikutantests mit einem 4\% wäßrigen Latexhandschuhextrakt (Hersteller: Allergopharma, Reinbek) durch. Dabei zeigten sich keine positiven Reaktionen ( $\mathrm{n}=178)$. Dieses entspricht dem Ergebnis einer Untersuchung, bei der keine (n=2002) epidermale Sensibilisierungen auf Gummilatex in den Konzentrationen von $2 \%$ und $4 \%$ beobachtet wurden (Gutgesell et al. 2000). In einer Publikation aus England wurden auf Gummilatex in 1,2\% ( $\mathrm{n}=822)$ positive Reaktionen gesehen (Wilkinson und Beck 1996). Im Unterschied zu unserer und zur Untersuchung von Gutgesell et al wurde hierbei nicht der ammonium-freie Gummilatex verwendet. Dies könnte die hohe Quote an irritativen und falsch positiven Reaktionen bei Wilkinson und Beck erklären (Gutgesell et al. 2000).

Ein epikutaner Test mit einem Naturgummilatexextrakt erscheint auf Grund der genannten Ergebnisse allenfalls gezielt, z.B. bei unklarer Handschuhallergie, sinnvoll. 


\section{Lyral (4-(4-Hydroxy-4-methylpentyl)-3-cyclohexen-1-carboxaldehyd)}

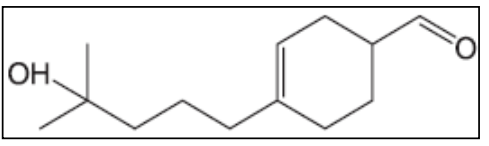

CAS Nr. 31906-04-4 (Le Coz et al. 2006)

Lyral, ein künstlicher Duft, gehört zu den am meisten verwendeten Duftstoffen in der Parfümerie (Frosch et al. 2006). In einer europäischen Untersuchung enthielten mehr als die Hälfte von 73 zufällig auf dem europäischen Markt (Deutschland, Schweden, England, Dänemark und Frankreich) zu kaufenden Deodorants und Parfüms Lyral (Rastogi et al. 1998). Üblicherweise wurden Duftstoffe mittels sog. Marker, wie den ersten Duftstoff-Mix, der acht Inhaltsstoffe (Zimtalkoholaldehyd, alpha-Amylcinnamylaldehyd, Eugenol, Isoeugenol, Hydroxycitronellal, Geraniol und Eichenmoos) beinhaltete und ursprünglich in den 70er Jahren der vorigen Jahrhunderts zusammengestellt wurde (Larsen 1977), regelmäßig in der Standardreihe getestet. Es zeigte sich jedoch, dass nicht alle Duftstoffallergien mit diesem Duftstoff-Mix I, entdeckt werden konnten (de Groot et al. 1993). So erfolgten im Rahmen einer multizentrischen Untersuchung der DKG parallel zur Standardreihe Epikutantestungen mit Lyral in einer Konzentration von 5\% (Vas.). In den unseren Tests zeigten sich in 3,9\% ( $n=337)$ positive Reaktionen. Das Gesamtergebnisses der DKG-Studie ergab eine Sensibilisierungsquote von 1,9\% $(\mathrm{n}=3185)$ mit der Konsequenz, dass Lyral im Jahre 2002 in die Standardreihe aufgenommen wurde (Geier et al. 2002a). In einer multizentrischen europäischen Untersuchung konnte für Lyral (5\%, Vas.) eine Sensibilisierungsquote von 2,7\% ( $\mathrm{n}=1855)$ beobachtet werden (Frosch et al. 1999), was die Aufnahme in die Standardreihe unterstütze. Auffällig hier war die regionale Variationsbreite der Quoten von 1,2\% in London bis $17 \%$ in Leuven, Belgien (Frosch et al. 1999).

Mittlerweile werden zwei Duftstoff-Mixe (Bestandteil von Mix I s.o.; Mix II: alphaHexylzimtaldehyd, Citral, Citronellol, Cumarin, Farnesol und Lyral) in der Standardreihe regelmäßig getestet, da gezeigt werden konnte, dass dadurch zusätzliche Sensibilisierungen entdeckt werden können (Frosch et al. 2005).

Die europäischen Sensibilisierungsraten vom Duftstoff-Mix II (beinhaltet Lyral) betragen zwischen 1,9\% $(\mathrm{n}=1845)$ in Spanien sowie 4,3\% $(\mathrm{n}=2694)$ in Deutschland und 7,9\% $(\mathrm{n}=678)$ in Österreich (Uter et al. 2012). 


\section{2-Mercaptobenzimidazol}

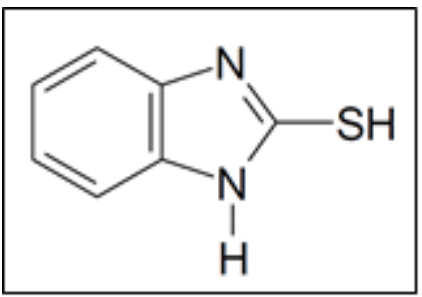

CAS Nr. 53988-10-6 (Sakemi et al. 2002)

2-Mercaptobenzimidazol (MBI) wird als Antioxidant (Handelsname u.a. Altofane MTB®) (Adams 1975) und Alterungsschutzmittel in Gummiprodukten (Fuchs 1995) eingesetzt. Durch berufliche Expostion wie z.B. beim Tragen von Gummistiefeln kann es zum Kontakt mit MBI kommen.

Mitte der 1990er Jahre wurde in einer Dortmunder Studie für MBI, bis dahin als seltenes Allergen beschrieben (Foussereau et al. 1983), eine Sensibilisierungsquote von 2,5\% (1\% in Vas., $\mathrm{n}=198$ ) publiziert (Geier et al. 1994). Außer einzelnen Fallberichten lagen keine weiteren Publikationen vor (Geier et al. 1994), so dass in Göttingen ab 1995 Tests parallel zur Standardreihe zur Abklärung dieser hohen Quote durchführten (1\% in Vas.). Hier wurden in 0,4\% ( $\mathrm{n}=231)$ positive Reaktionen beobachtet. Weitere Studien mit Angaben zu Sensibilisierungsquoten sind unseres Wissens nicht bekannt. Aus diesem Ergebnis lässt sich keine Empfehlung zur regelmäßigen Testung ableiten. Jedoch können die Dortmunder Schlussfolgerungen, dass MBI häufiger als angenommen zu Sensibilisierungen führen kann (Geier et al. 1994), nicht widersprochen werden, so dass zumindest gezielte Testungen bei Verdacht auf eine entsprechende Gummiallergie sinnvoll erscheinen.

\section{Mercaptobenzothiazol}

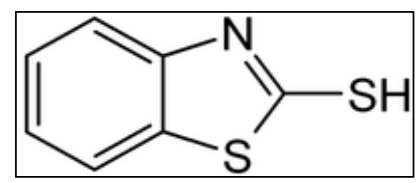

CAS Nr. 149-30-4 (Le Coz et al. 2006)

Mercaptobenzothiazol (MBT) wird in der Gummiindustrie als Vulkanisationsbeschleuniger und Alterungsschutzmittel (Fuchs 1995) eingesetzt und findet Verwendung in Handschuhen, Reifen, Schuhsohlen, Kabeln aber auch als Fungizid in der Agrarindustrie. Seit den 1960er Jahren wurde ein allergisches Potential von MBT und seinen Derivaten beschrieben (Fuchs 1995). Als Marker wurde bis Mitte der 1990er Jahre der sog. Mercapto-Mix in die Standardreihe der DKG eingesetzt, bestehend aus 4 Komponenten in gleichen Konzentrationen: N-cyclohexyl-2-benzothiazylsulfenamid (CBS), Dibenzothiazyldisulfid 
(MBTS), Morpholinylmercaptobenzothiazol (MOR) und dem MBT. Auswertungen von IVDK Daten zeigten, dass dieser Mix keine befriedigende Sensitivität hatte (Geier und Gefeller 1995). Darüber hinaus gab es Hinweise für chemische Instabilitäten bezüglich der Konzentrationen in der vier Komponenten Mischung (Geier et al. 2006a). Frühere Untersuchungen mit MBT (2\% in Vas.) zeigten in Deutschland eine Sensibilisieungsquote von 0,9\% ( $\mathrm{n}=851$ ) (Fuchs 1995). Ab dem Jahr 1995 kam ein neuer Mercapto-Mix, bestehend aus 3 Komponenten (CBS, MBTS und MOR), ohne MBT, in die Standardreihe zur Testung. In Göttingen führten wir im Rahmen einer multizentrischen Untersuchung der DKG Testungen parallel zur Standardreihe mit MBT in 2\% Konzentration (Vas.) durch und beobachteten in $0,8 \% \quad(n=2044)$ positive Reaktionen. Die Sensibilisierungsrate der Gesamtpopulation des IVDK betrug 1,8\% ( $\mathrm{n}=7198)$ (Geier et al. 2002b). In dieser Studie konnte gezeigt werden, dass eine Testung des neuen Mercapto-Mix allein nicht zu einer genügenden Detektionsrate führte, jedoch in Kombination mit MBT diese Rate deutlich erhöht werden konnte (Geier et al. 2002b).

Multizentrische finnische Studien zeigten für MBT (2\% in Vas.) Sensibilisierungsquoten von 0,5\% ( $\mathrm{n}=11840)$ (Hasan et al. 2005). In Nordamerika kam seit dem Jahre 1977 der verkleinerte Mercapto-Mix und MBT getrennt zum Einsatz (Lynde et al. 1982). Die Quoten für MBT (1\% in Vas.) betrugen 2,7\% $(\mathrm{n}=5732)$ in einer multizentrischen Untersuchung (Lynde et al. 1982), 1,0\% ( $\mathrm{n}=1039)$ bei einer Konzentration von 1\% (Vas.) und 0,7\% bei einer Konzentration von 2\% (Vas.) im Rahmen einer Studie der Mayo-Clinic (Wetter et al. 2005). Die NACDG publizierte Quoten für eine Konzentration von 1\% (Vas.) von 1,8\% ( $\mathrm{n}=3440)$ (Marks et al. 2000) und 2,0\% ( $\mathrm{n}=5834)$ (Marks et al. 2003).

Seit 1995 erfolgte die Aufnahme von MBT in die Standardreihe (Geier et al. 2002b), mit der Empfehlung die Diagnostik mit einer Testkonzentration von 2\% (Vas.) parallel zum neuen Mercapto-Mix durchzuführen (Diepgen et al. 2006). Dies scheint in der aktuellen Diskussion weiterhin die gängige Meinung zu sein (Uter et al. 2016). Die Sensibilisierungsquoten im Rahmen der Standardreihe der DKG betrugen 0,5\% ( $\mathrm{n}=8536)$ (Schnuch et al. 2005c) bzw. 0,6\% ( $\mathrm{n}=30705)$ (Worm et al. 2005). Erhebungen der europäischen Standardreihe, in der MBT (2\% in Vas.) ebenfalls parallel mit dem Mercapto-Mix zum Einsatz kommt, betrug die Quote 1,0\% (Bruynzeel et al. 2005). Aktuelle europäische Daten zeigten Raten zwischen 0,2\% $(n=670)$ in Litauen, 0,7\% $(n=2694)$ in Deutschland und 1,4\% $(n=2402)$ in der Schweiz (Uter et al. 2012). Zuletzt wurden Quoten von 0,79\% $(\mathrm{n}=28141)$ in einer multizentrischen Auswertung publiziert (Uter et al. 2016). 
Für MBT sind darüberhinaus karzinogene Eigenschaften ermittelt worden (Drexler und Broding 2007).

\section{Myristylalkohol}

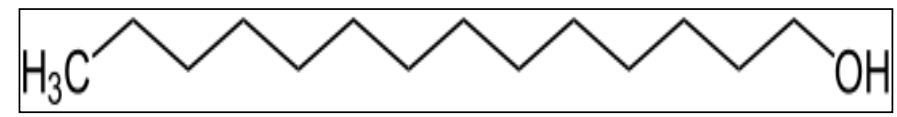

CAS Nr. 112-72-1 (Corberan et al. 2014)

Myristylalkohol (MA) (1-tetradecanol) wird als Weichmacher und Lösungsmittel in Kühlschmierstoffen verwendet (Geier et al. 2006b). Weiterhin ist es ein Zusatzstoff in Kosmetika (de Groot et al. 1988). In Göttingen untersuchten wir MA in einer Konzentration von $10 \%$ (Vas.). Dabei beoachteten wir in $7,1 \%$ positive Reaktionen, jedoch bei einer geringen Patientenzahl $(n=26)$. Hinweise für ein problematisches Allergen zeigten sich in einer typischen Konstellation mit häufig fraglichen und schwach positiven Reaktionen und einem negativen RI (-0.4), einem Indiz für falsch positive Reaktionen (Geier et al. 2003d). In einer multizentrischen Studie der DKG, um Sensibilisierungsquoten bei Kühlschmierstoffen $\mathrm{zu}$ erheben, zeigten sich für MA in einer Konzentration von 10\% (Vas.) in 2,8\% (n=128) positive Reaktionen mit häufig fraglichen und schwach positiven Reaktionen (Geier, et al. 2006). In einer anderen deutsch-dänischen Untersuchung wurde bei MA (10\% in Vas.) eine Sensibilisierungsrate von $7 \%(\mathrm{n}=90)$ bei einer ähnlichen Konstellation mit einem hohen Anteil an fraglichen und einfach positiven (+) Reaktionen und einem negativen RI $(-0.7)$ beobachtet (Geier et al. 2006b). Diese Ergebnisse ließen vermuten, dass eine Testkonzentration von 5\% (Vas.) anstatt 10\% (Vas.) sinnvoll sein könnte, wie bereits in einer italienischen Studie untersucht. Die Quote lag hier bei 2,1\% $(n=146)$ mit wenigen Hinweisen auf irritative Reaktionen (Tosti et al. 1996). Weitere Publikationen bezüglich Sensibilisierungsquoten liegen nach unseren Recherchen nicht vor. $\mathrm{Zu}$ beachten ist, dass in der Regel keine klinischen Beschwerden im Falle einer positiven Reaktion vorlagen (Geier et al. 2006b). Um MA zukünftig besser beurteilen zu können, sollte zunächst die optimale Testkonzentration bestimmt werden. Evtl. könnten Tests parallel zur Standardreihe z. B. im Kühlschmierstoff- oder im Externa-Block der DKG in Erwägung gezogen werden. Zumindest sollte MA gezielt nach anamnestischen Hinweisen getestet werden. 


\section{Natriumdisulfit}

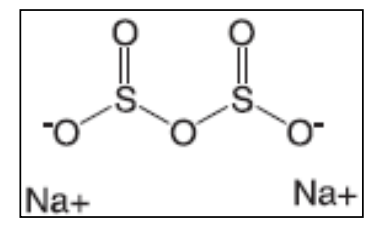

CAS Nr. 7681-57-4 (Le Coz et al. 2006)

Natriumdisulfit (Natriumpyrosulfit oder Natriummetabisulfit) wird als Antioxidant und Konservierungsmittel in oralen, parenteralen und topischen Arzneimitteln eingesetzt (Madan et al. 2007). Bereits in der Zeit vor Christus wurden in China Sulfite als Konservierungsmittel für Lebensmittel verwendet, und bis heute ist Natriumdisulfit als Lebensmittelzusatzstoff E223 zugelassen (Przybilla und Ring 1987). Weitere Verwendung findet es als Stabilisator in der Fotoindustrie (Riemersma et al. 2004). Auf Grund von publizierten epidermalen Sensibilisierungen unklarer Relevanz (Madan et al. 2007) führten wir Testungen parallel zur Standardreihe durch. Dabei konnten wir in 1,3\% ( $\mathrm{n}=2635)$ positive Reaktionen auf Natriumdisulfit (1\% Vas.) beobachten. Aus Italien wurde eine Sensibilisierungsquote von 1,7\% ( $\mathrm{n}=2894)$ publiziert (Vena et al. 1994). In einer britischen Untersuchung wurde bei gleicher Konzentration (1\% in Vas.) eine Quote von 4,1\% (N=1751) berichtet (Madan et al. 2007). In einer schwedischen Studie wurde bei Natriumdisulfit in einer Konzentration von 2\% (Vas.) eine Quote von 3,4\% ( $\mathrm{n}=1518)$ beobachtet (Kaaman et al. 2010). Hinweise auf irritative bzw. falsch positive Reaktionen in der Testkonzentration von 1\% (Aqua) (AaltoKorte et al. 2009) haben zu einer Diskussion bezüglich des Testvehikels und der Testkonzentration geführt (Madan und Beck 2009), wobei derzeit als Vehikel Vaseline empfohlen wird (Kaaman et al. 2010). Englische Untersuchungen berichteten zuletzt über eine Rate von 3,8\% $(\mathrm{n}=183)$ in $1 \%$ (Petr.) (Oliphant et al. 2012). Eine retropsektive Auswertung einer Hautarztpraxis zeigte eine Quote von 3,1\% ( $\mathrm{n}=780)$, jedoch wurde die klinische Relevanz dieser Sensibilisierungen bei mehr als die Hälfte der Patienten in Frage gestellt (Häberle et al. 2016).

Aktuell ist Natriumdisulfit lediglich im Ophtalmika-Block der DKG enthalten. Auf Grund der genannten Ergebnisse scheint eine gezielte anamnesebezogene Testung sinnvoll. Eine regelmäßige Testung darüberhinaus wird aktuell auf Grund der geringen klinischen Relevanz trotz der weiten Verbreitung dieser Substanz für nicht sinnvoll erachtet (Häberle et al. 2016). 


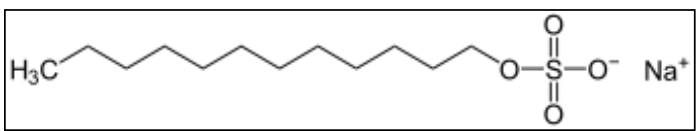

CAS Nr. 151-21-3 (Le Coz et al. 2006)

Natriumlaurylsulfat ist ein Tensid, welches in u.a. Shampoos, Waschmittel, Zahncremes und Schaumbäder eingesetzt wird (Lee und Maibach 1995) und ein bekanntes Hautirritans (Frosch et al. 2006). In Göttingen testeten wir im Rahmen einer multizentrischen Studie der DKG drei Konzentrationen $(0,25 \%, 0,5 \%$ und $1 \%$ in Vas.) um Sensibilisierungs- und Irritationsraten festzustellen. Dabei zeigte sich eine proportional zur Konzentration ansteigende Anzahl an irritativen Reaktionen bei geringem Anstieg von allergischen Reaktionen mit Quoten von $0,1 \%, 0,1 \%$ und $0,4 \%(\mathrm{n}=1413$, jeweils in Vas.) bei einem stark negativen RI ( $-0,9$ bis -1.0$)$, einem Indiz für falsch positive (Geier et al. 2003d). Natriumlaurylsulfat bietet eine diagnostische Hilfe, um vor allem schwach positive (+) allergische Reaktionen gegenüber irritativen Reaktionen differenzieren zu können und eine simultane Testung wird bei allen Allergentestungen empfohlen (Tupker et al. 1997) und ist in der Standardreihe vertreten. Die Empfehlungen weisen daruf hin, dass das optimale Testvehikel wäßrig (Aqua) sein sollte (Löffler et al. 1998) bei einer Testkonzentration von 0,25\% (Becker 2013). Interessant ist die Beoabachtung, dass die Reaktionsquoten abhängig von der Jahreszeit sein können. Im Winter und Frühling wurden im Vergleich zum Sommer und Herbst signifikant häufiger irritative Reaktionen beobachtet (Geier et al. 2003b).

\section{Phenoxyethanol}

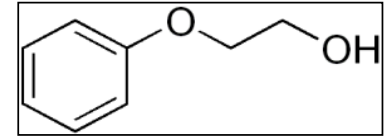

CAS Nr. 122-99-6 (Le Coz et al. 2006)

2-Phenoxyethanol (PE), ein breit angewandtes Konservierungsmittel mit bakterizidem Wirkungsspektrum, wird industriell zur Konservierung von Harzen, Farbtinte und in Kühlschmierstoffen eingesetzt (Hausen 1993a). Weitere Verwendung findet es in Desinfektions- und Lösungsmitteln sowie in Kosmetika wie Hautpflegeprodukten und Deodorants. PE ist mit 80\% Hauptbestandteil vom Euxyl ${ }^{\circledR}-\mathrm{K}-400$ (Hausen 1993a), welches eines der meist gebräuchlichen Konservierungsmittel für Kosmetika in Deutschland war. Seit Anfang der 80er Jahre fand Euxy $1^{\circledR}-\mathrm{K}-400$ Verwendung in Produkten wie z.B. Shampoos, Seifen, Badezusätze und Gesichtscremes (Hausen 1993a). Die starke Zunahme der Verwendung führte ebenfalls zu einem Anstieg von Sensibilisierungen, weswegen es im Jahre 1991 in die Standardreihe der DKG aufgenommen wurde (Geier et al. 1996a). Die zweite 
Komponente des $\operatorname{Euxyl}^{\circledR}-\mathrm{K}-400$ ist mit einem Anteil von 20\% Dibromidcyanobutan (=Methyldibromoglutaronitril oder MDBGN). Während eine stetige Zunahme von Sensibilisierungen gegenüber MDGBN beobachtet wurden, waren Reaktionen gegenüber Phenoxyethanol Einzelfälle (Fuchs et al. 1991). Wir testeten Phenoxyethanol (1\% in Vas.) im Rahmen einer multizentrischen Studie der DKG, um einen Eindruck über die Sensibilisierungsrate zu bekommen. Dabei zeigten sich in $0,5 \%(n=554)$ positive Reaktionen. Im Rahmen einer früheren großen multizentrischen Studie der 1990er Jahre wurde eine Sensibilisierungsrate (1\% in Vas.) von $0,1 \%(\mathrm{n}=11120)$ gesehen (Schnuch et al. 1998). In Italien wurde im Jahre 2005 über eine PE eine Sensibilisierung in Folge des Einsatzes eines topischen Medikaments berichtet (Gallo et al. 2005). Eine frühere italienische Studie (5\% in Vas.) zeigte eine Quote von 0,1\% ( $\mathrm{n}=3433)$ (Tosti et al. 1995).

PE kann auf Grund der o.g. Ergebnisse als ein seltenes Allergen angesehen werden. Seit dem Jahre 2005 wurde Euxyl ${ }^{\circledR}-\mathrm{K}-400$ aus der Standardreihe der DKG gestrichen und durch MDBGN (siehe Seite 36) als das wesentliche Allergen ersetzt (Geier und Mahler 2016).

Nach gezielten Tests auf PE bei entsprechenden anamnestischen Hinweisen, z.B. bei Drehern, konnte eine Sensibilisierungsquote von 0,5\% ( $\mathrm{n}=199)$ beobachtet werden (Geier et al. 2004). PE ist aktuell im Kühlschmierstoff-Block der DKG vertreten und zumindest eine gezielte Untersuchung erscheint sinnvoll.

In diesem Zusammenhang erscheint nennenswert, dass in Nordamerika weiterhin die Kombination von MDBGN/PE getestet wird (Zug et al. 2009).

\section{Poly(hexamethylendiguanid)-HCL}

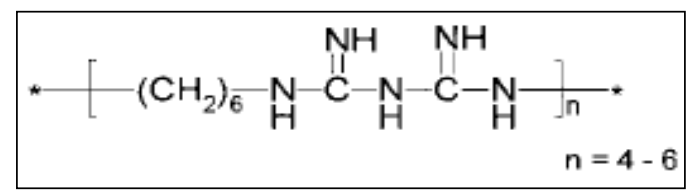

CAS Nr. 28 757-47-3 (Schnuch et al. 2000)

Polyhexamethylendiguanid-HCl entspricht dem Salz von Polyhexamethylenbiguanid (PHMB) und wird wegen seinen antimikrobiellen Eigenschaften als Konservierungsmittel in Kosmetika, medizinischen Produkten wie Kontaktlinsen- und Wundspüllösungen (Schnuch et al. 2000) sowie als Desinfektionsmittel in Schwimmbädern eingesetzt (Schnuch et al. 2007a). PHMB untersuchten wir im Rahmen einer multizentrischen Studie des IVDK parallel zur Standardreihe in einer Konzentration von 2,5\% jeweils gelöst in Aqua und Vaselin. Dabei beoachteten wir in 0,5\% ( $\mathrm{n}=651$, Aqua) und $0,2 \%(\mathrm{n}=464$, Vas.) positive Reaktionen. Ähnliche Sensibilisierungsquoten von $0,5 \%$ (2,5\% in Aqua) und 0,8\% (5\% in Aqua) (n=1975) wurden in der IVDK-Gesamtpopulation festgestellt (Schnuch et al. 2007a). Eine frühere 
IVDK-Studie zeigte eine Quote von 0,4\% ( $\mathrm{n}=1554)$ für eine Testkonzentration von 2,5\% (Aqua) (Schnuch et al. 2000). Die Daten weisen daraufhin, dass PHMB trotz der vielseitigen Anwendungen ein seltenes Allergen zu sein scheint. Regelmäßige Testungen sind derzeit nicht sinnvoll, so dass allenfalls gezielt anamnesebezogene Testungen in Erwägung gezogen werden sollten.

\section{Prednicarbat}

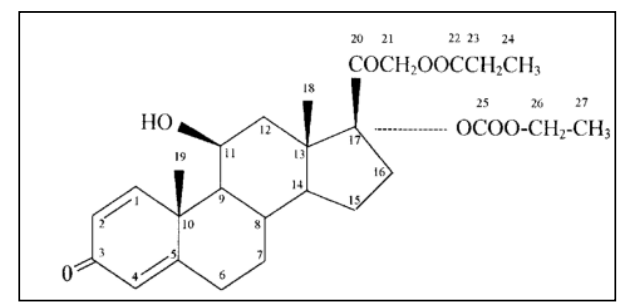

CAS Nr. 73771-04-7 (Stingeni und Lisi 1999)

Prednicarbat (P) ist ein mittelstark wirksames Glukokortikosteroid zur topischen Anwendung auf der Haut (Dunkel et al. 1991) und wird insbesondere zur Behandlung der atopischen Dermatitis und Psoriasis eingesetzt (Gupta und Chow 2004). Die Einteilung der topisch anwendbaren Glukokortikosteroide in verschiedenen Klassen richtet sich entweder nach ihrer Wirkstärke (Niedner 1996) oder nach der hier verwendeten Klassifikation, die chemische und allergologische Gesichtspunkte berücksichtigt. Demnach gibt es 4 (A-D) allergene HauptGruppen (Matura und Goossens 2000), wobei Prednicarbat (wie z.B. Hydrocortison-17butyrat) in die Gruppe D eingeteilt wird, d.h. langkettiger Ester an C17 und/oder C21.

Seit den 1950er Jahren werden topische Glukokortikosteroide gegen entzündliche und proliferative Hauterkrankungen eingesetzt und haben den Vorteil, dass sie eine relativ geringe systemische Wirkung und Toxizität haben (Isaksson 2004). Bei einer Kontaktallergie auf Glukokortikoide besteht grundsätzlich die Möglichkeit, dass durch Fehlinterpretation die Allergie maskiert wird (Gonul und Gul 2005). Als Nebenwirkung einer Langzeittherapie ist vor allem die Hautatrophie zu nennen. Des Weiteren kann es zu einer Unterdrückung der Hypothalamus-Hypophysen-Achse kommen, die $\mathrm{zu}$ einer Störung der endogenen Kortisonausschüttung führen kann (Schäfer-Korting et al. 1993).

Prednicarbat ist bekannt für ein positives Risiko/Nutzen Verhältnis zwischen Wirkung und Nebenwirkung (Schäfer-Korting et al. 1993) und wird deshalb häufig als Glukokortikosteroid der Wahl bei der Behandlung von Kindern, älteren Patienten und als Langzeittherapie eingesetzt (Gupta und Chow 2004).

Prednicarbat zeigte in den unseren Epikutantestungen mit den Testkonzentrationen von 0,1\% (Vas.) und 0,25\% (Vas.) jeweils in $0,3 \%$ ( $n=2385$ und $n=3200)$ positive Reaktionen. Eine 
holländisch/ belgische Untersuchung zeigte für eine Testkonzentration von 1\% (Eth.) Quoten von 0,65\% ( $\mathrm{n}=533)$ bzw. 0,4\% ( $\mathrm{n}=610)$ (Dooms-Goossens et al. 1994). Italienische Studien verglichen Sensibilisierungsquoten von 1\% Prednicarbat in Vaseline bzw. Ethanol und beobachteten bei jeweils 0,62\% ( $n=325)$ positive Reaktionen (Stingeni und Lisi 1999).

Routinemäßige Tests von Prednicarbat in der Standardreihe sind auf Grund der o.g. Ergebnisse derzeit nicht zu empfehlen. Gezielte anamnesebezogene Epikutantests erscheinen ausreichend. Als Marker für Prednicarbat ist Budenosid, bereits vertreten in der DKG Kortkoidreihe, beschrieben (Goossens et al. 2000)

\section{Propolis}

Propolis ist ein Bienenharz mit einer Zusammensetzung von etwa 55\% Naturharz, 30\% Wachs, 10\% ätherischen Ölen und 5\% Pollen (Hausen et al. 1992), und bereits im alten Ägypten wurde Propolis zur Einbalsamierung von Mumien verwendet (Ghisalberty 1979).

Wegen anti-inflammatorischen und anästhesiologischen Eigenschaften kann es gegen Akne und Psoriasis als Antibiotikum und Antimykotikum eingesetzt werden (Hausen et al. 1987). Bei Imkern sind Propolisallergien als Berufskrankheit anerkannt (Hausen et al. 1987) und erste Beobachtungen eines Kontaktekzems auf Propolis reichen bis ins Jahr 1915 zurück (Hausen et al. 1987).

Propolis als Naturheilmittel durch Einsatz in vielen Naturkosmetika vermehrt in Mode gekommen (Aberer et al. 2003). Dies könnte ein Grund für eine stetige Zunahme des Kontaktekzems auf Propolis in den letzten Jahren sein (Giusti et al. 2004). In Europa konnten als Hauptallergene Kaffesäureester identifiziert werden (Hausen 2005), daneben kommen Ester der Benzoesäure, der Ferulasäure und der Isoferulasäure als Sensibilisatoren in Betracht (Aberer et al. 2003). In Göttingen führten wir im Rahmen einer multizentrischen Studie der DKG parallel zur Standardreihe Tests mit 10\% Propolis in Vas. durch und beobachteten in 1,7\% ( $\mathrm{n}=1606)$ positive Reaktionen. Die Gesamtpopulation der DKG zeigte eine Sensibilisierungsquote von ebenfalls $1,7 \%$ (10\% in Vas., $n=3309)$ (Uter et al. 2005). Diese Ergebnisse führten dazu, dass Propolis 10\% in Vas. ab 1999 in die Standardreihe aufgenommen wurde, wo Sensibilisierungsquoten von 2,2\% ( $\mathrm{n}=8531)$ (Schnuch et al. 2005c) und 2,3\% ( $n=31029)$ (Worm et al. 2005) beobachtet wurden. In Finnland wurde im Zeitraum von 1995 bis 2002 ein Anstieg der Quote von 0,5\% (n=3885) auf 1,4\% (n=5130) (10\% Vas.) publiziert (Hasan et al. 2005). 
Beachtenswert ist die hohe Sensibilisierungsquote von 4,9\% (20\% in Vas., $\mathrm{n}=1255$ ) bei Kindern auf Propolis in einer italienischen Studie mit der Schlussfolgerung der Autoren, dass Propolis nicht in topischen Produkten für Kinder verwendet werden sollte (Giusti et al. 2004).

\section{Propylenglykol}

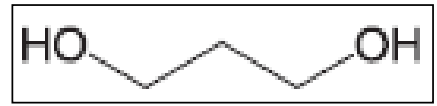

CAS Nr. 57-55-6 (Le Coz et al. 2006)

Propylenglykol (PG) ist ein flüssiger Trägerstoff, der mit Ethanol und Wasser gut mischbar ist (Andersen und Storrs 1982). PG ist farblos, fast geruchlos und ein häufig eingesetzter Bestandteil von Lösungsmitteln, da es durch die Verbesserung der Löslichkeit zu einer höheren Resorption von vielen Substanzen führen kann (Andersen und Storrs 1982). Auf Grund weiterer antimikrobieller Eigenschaften von PG brauchen oft keine Konservierungsmittel eingesetzt werden (Andersen und Storrs 1982). Verwendet wird PG in Pharmaka, Nahrungszusatzstoffen (z.B. Farb- und Geschmacksverstärkern), Waschmitteln oder als Befeuchtungsmittel in Zigaretten (Aberer et al. 1993). Anfang der 1980er Jahre war PG in bis zu 30\% aller Kosmetika in den USA enthalten (Maibach et al. 1980). Industriellen Einsatz findet PG in Lösemitteln, Farbtinten und Lacken (Aberer et al. 1993). Anfang der 1990er Jahre wurde im Rahmen einer multizentrischen Studie der DKG versucht, nach wiederholten Fallberichten über Sensibilisierungen (Aberer et al. 1993), die optimale Testkonzentration für PG zu ermitteln. Es wurde berichtet, dass es wegen häufigen irritativen Reaktionen schwierig war, eine genaue Differenzierung gegenüber allergischen Reaktionen vorzunehmen (Aberer et al. 1993). Als Konsequenz erfolgte die Empfehlung, PG in 20\% (Aqua) zu testen (Aberer et al. 1993). Im Rahmen einer späteren multizentrischen Studie der DKG testeten wir PG 5\% in Vas. und 20\% in Aqua parallel zur Standradreihe und beobachteten in $0,2 \%(n=3214)$ bzw. in 2,0\% $(n=611)$ positive Reaktionen. Auffällig waren häufig fragliche sowie höchstens einfach positive (+) Reaktionen. Dies spiegelt sich im negativen RI (jeweils -0.6) wider, einem Indiz für falsch positive oder irritative Reaktionen (Geier et al. 2003d).

Finnische Studien zeigten im Zeitraum zwischen 1995 und 2002 Sensibilisierungsquoten von 0,3\% $(\mathrm{n}=5270)$ und $0.2 \%(\mathrm{n}=6875)$ bei nicht angegebener Konzentration (Hasan et al. 2005). Die NACDG aus Nordamerika publizierten Quoten von 3,5\% ( $\mathrm{n}=4454)$ (30\% in Vas.) (Warshaw et al. 2009) und 3,7\% (n=5804) (10\% in Vas.) (Marks et al. 2003) sowie 3,8\% $(\mathrm{n}=4095)$ (30\% in Aqua) (Marks et al. 2000). Eine Untersuchung aus der Mayo-Clinic in den USA zeigte eine Quote von 0,8\% (10\% in Vas.) (n=711) (Wetter et al. 2005). Eine türkische 
Untersuchung wiederum zeigte keine allergischen Reaktionen (5\% in Vas.) $(n=308)$ (Boyvat et al. 2005). Die Sensibilisierungsquote der IVDK Gesamtpopulation lag bei 2,3\% (20\% in Aqua, $n=45138$ ) mit der Beobachtung von häufig fraglichen sowie höchstens einfach positiven (+) Reaktionen (RI -0.14) (Lessmann et al. 2005). Eine aktualisierte Empfehlung nach 2005 bezüglich einer optimalen Testkonzentration liegt unserer Kenntniss nach nicht vor. Man könnte in Erwägung ziehen, allenfalls positive Reaktionen mit PG 5\% (Vas.) nachzutesten, um potentiell irritative Reaktionen zu überprüfen.

Derzeit wird PG in 20\% (Aqua) im Externa Block der DKG getestet. Auf Grund des breiten Einsatzes von PG und den oben genannten Ergebnissen erscheint eine regelmäßige Testung parallel zur Standardreihe sinnvoll.

\section{Quaternium-15}

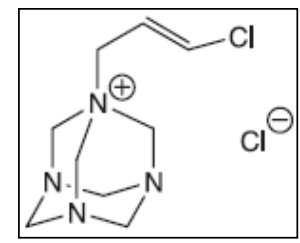

CAS Nr. 4080-31-3 (Le Coz et al. 2006)

Quaternium-15 (N-(3-chlorallyl) hexaminium Chlorid) wird auf Grund bakterizider Eigenschaften als Konservierungsmittel in vielen Produkten, wie Shampoos, Gesichtskosmetik, Feuchtigkeitslotionen und Sonnenmilch eingesetzt (Zina et al. 2000).

Es besitzt zudem als sog. Formaldehydabspalter die Eigenschaft in Anwesenheit von Wasser die grösste Menge an Formaldehyd in dieser Gruppe freisetzen zu können und ist eines der meist verwendeten sensibilisierenden Konservierungsmittel in den USA (Fuchs 2007b).

In Göttingen nahmen wir Tests parallel zur Standardreihe vor und zeigten in 0,6\% $(n=2635)$ positive Reaktionen (1\% in Vas.). In Australien und Nord Amerika, wo Quaternium 15 ein häufig verwendetes Produkt ist, fanden sich unterschiedlich hohe Sensibilisierungsquoten (zwischen 1\% - 9\%) (Cahill und Nixon 2005). Aus Nordamerika liegen mehrere Sensiblisierungsquoten vor: In der Mayo-Clinic wurde eine Quote von 5,1\% für 2\% (Vas., $\mathrm{n}=408$ ) sowie eine Quote von 7,8\% ( $\mathrm{n}=913$ ) für 1\% (Vas.) beobachtet (Wetter et al. 2005). Die NACDG berichtete über eine stetige Zunahme der bereits hohen Quoten. Im Jahre 2000 wurde eine Quote von 9,0\% (2\% Vas., n=3436) publiziert (Marks et al. 2000), diese stieg 2003 auf 9,2\% (2\% Vas., n=5832) (Marks et al. 2003) und 2009 wurde eine Quote von 10,3\% (2\% Vas., $\mathrm{n}=4454$ ) publiziert (Zug et al. 2009).

In Italien zeigte sich zu Beginn der 1990er Jahre eine Rate von 0,2\% (1\% Vas., n=4470) (Tosti et al. 1990). In einer schweizer Untersuchung Mitte der 1990er Jahre betrug die Quote 
$1,0 \%$ (1\% in Vas., $\mathrm{n}=2295$ ) (Perrenoud et al. 1994), britische Studien (1\% in Vas.) berichteten über Quoten von 1,9\% ( $\mathrm{n}=6958)($ Jong et al. 2007) und 1,8\% $(\mathrm{n}=5167)$ (Jacobs et al. 1995). In türkischen Tests betrug die Quote 0,6\% (1\% Vas., $\mathrm{n}=308)$ (Boyvat et al. 2005). Multizentrische finnische Studien fanden Quoten von 1,1\% ( $n=9364)$ und 0,8\% ( $n=11802)$ bei nicht angegebener Konzentrationen (Hasan et al. 2005). Quaternium 15 (1\% in Vas.) ist in der Europäischen Standardreihe vertreten und zeigte dort eine Sensibilisierungsrate von 1,3\% $(n=26210)$ (Bruynzeel et al. 2005).

Die oben genannten unterschiedlichen Quoten vermitteln den Eindruck, dass unterschiedliche regionale Einflüsse, wie die Verwendung von verschiedenen Kosmetika, eine wichtige Rolle spielen könnten. Diesen Eindruck bestätigte eine europäische Untersuchung wo Raten zwischen $0,1 \%(\mathrm{n}=1676)$ in Italien über 1,2\% $(\mathrm{n}=4013)$ in Spanien und den Niederlanden bis hin zu 3,2\% ( $\mathrm{n}=760)$ in Finnland beobachtet wurden (Uter et al. 2012).

Quaternium-15 ist im Konservierungsblock der DKG enthalten. Auf Grund der gezeigten Ergebnisse sollte eine Untersuchung zumindest gezielt bei anamnestischem Verdacht auf Kosmetikaallergie erfolgen.

\section{Sesquiterpenlactone-Mix}

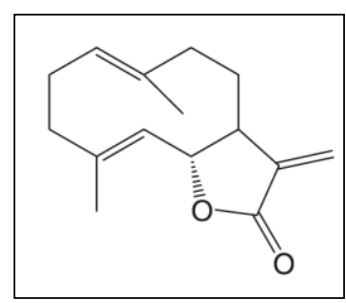

Costunolid: CAS Nr. 553-21-9

(Le Coz et al. 2006)

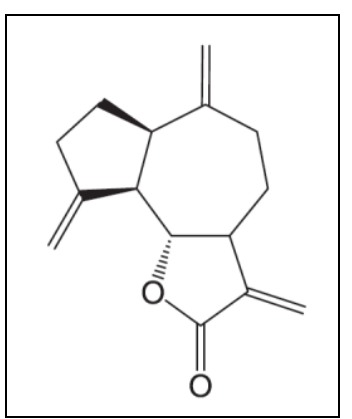

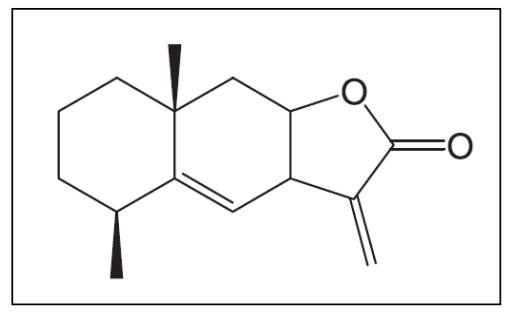

Alantolakton: CAS Nr. 546-43-0

(Le Coz et al. 2006)

Dehydrocostuslakton: CAS Nr. 477-43-0 (Le Coz et al. 2006)

Der Sesquiterpenlactone-Mix (SL) besteht aus drei pflanzlichen Substanzen: Costunolid, Alantolakton und Dehydrocostuslakton (Aberer et al. 2003). Diese Substanzen kommen insbesondere in Blumen der Familie der Kompositen (Korbblütler) vor (Paulsen 1992). Bekannte Sorten dieser Blumen sind Arnika und Kamille. Wegen ihren 
entzündungshemmenden Eigenschaften werden diese u. a. als Arzneimittel für die Haut in Cremes, Salben und Naturkosmetika eingesetzt (Paulsen 1992). Wegen vermeintlich zunehmender Kontaktallergien auf Kompositen (Ducombs et al. 1990) führten wir in Göttingen Epikutantests parallel zur Standardreihe durch und beobachteten in 1,1\% ( $\mathrm{n}=2629)$ positive Reaktionen bei einer Konzentration von $0,1 \%$ (Vas.). In einer multizentrischen europäischen Studie wurde ebenfalls eine Sensibilisierungsrate von 1,1\% (0,1\% in Vas., $\mathrm{n}=26210$ ) berichtet (Bruynzeel et al. 2005). Eine multizentrische finnische Studie zeigte Quoten von $0,6 \%(0,1 \%$ in Vas., $n=5965)$ (Hasan et al. 2005) und eine dänische Studie publizierte eine Quote von 2,8\% (0,1\% in Vas., $n=4386)$ (Paulsen et al. 2001). Nordamerikanische Untersuchungen zeigten mit dem SL Mix in jeweils einer Konzentration von $0,1 \%$ (Vas.) Quoten von 1,1\% ( $\mathrm{n}=708)$ in der Mayo-Clinic (Wetter et al. 2005) sowie 0,9\% ( $\mathrm{n}=5800)$ (Marks et al. 2003) und 0,7\% ( $\mathrm{n}=4095)$ (Marks et al. 2000) bei der NACDG. In britischen Studien (jeweils $0,1 \%$ in Vas.) wurden Quoten von 2,1\% ( $n=656)$ (von der Werth et al. 1999) und 1,1\% $(\mathrm{n}=3062)$ (Britton et al. 2003) publiziert.

Trotz der hohen Sensibilisierungsraten erscheint eine Aufnahme des SL-Mix in die Standardreihe nicht sinnvoll, da Tests mit SL-Mix nicht die sensitivste Methode darstellen, um Kompositen-Allergien zu entdecken (von der Werth et al. 1999). Bei einem Vergleich mit dem sog. Kompositen-Mix, welches Kurzetherextrakte der 5 Kompositenpflanzen Arnika, Kamille, Schafgarbe, Rainfarn und Mutterkraut enthält (Aberer et al. 2003), wurden bis zu $50 \%$ niedrigere Detektionsraten festgestellt (von der Werth et al. 1999). Eine weitere finnische Studie bekräftigte, dass eine Testung vom SL-Mix keinen Vorteil gegenüber dem Kompositen-Mix besitzt (Kanerva et al. 2001).

Durch die routinemäßige Testung vom Kompositen-Mix im Standard der DKG ist eine regelmäßige Testung des SL-Mix nicht notwendig, jedoch erscheint es meines Erachtens sinnvoll, den SL-Mix bei gezieltem Verdacht auf eine Pflanzen Allergie ergänzend zu testen.

\section{Tantalum und Tantalum-V-Chlorid}

Tantalum ist ein Metall und wird als das 73. Element im Periodensystem der Elemente aufgeführt. Dessen Metallsalz Tantalum-V-Chlorid findet vor allem industriell in Kondensatoren, die in der Automobilindustrie aber auch in Mobiltelefonen eingesetzt werden, verwendung (Werman und Rietschel 1981). Seit den 1940er Jahren werden diese Substanzen auch in medizinischen Instrumenten und Implantaten eingesetzt (Werman und Rietschel 1981). Dabei macht man sich die Eigenschaft zu Nutze, dass Tantalum nicht mit Körperflüssigkeiten und Körpergeweben reagiert und äußerst hitzebeständig ist (Werman und Rietschel 1981). Auf Grund von Fallberichten aus Spanien über Kontaktallergie nach einer 
Metallosteosynthese sowie zunehmenden Einsatzes von Tantalum und dessen Metallsalz in Implantaten (Romaguera und Vilaplana 1995), wie z.B. Hüftprothesen, führten wir Tests mit verschiedenen Konzentrationen von Tantalum und Tantalum-V-Chlorid jeweils in Vaseline durch. Dabei zeigte Tantalum in Konzentrationen von $0,1 \%(n=78)$ und $1 \%(n=370)$ keine positiven Reaktionen. Bei Tantalum-V-Chlorid beobachteten wir in Konzentrationen von $0,1 \%$ und $1 \%$ in $0 \%(n=1947)$ bzw. $0,3 \%(n=2783)$ positive Reaktionen. Weitere Studien mit Sensibilisierungsquoten liegen nicht vor. In zwei Publikation aus den USA wurde über Urtikaria nach Einsatz mit Tantalum legierten chirurgischen Clips berichtet (King et al. 1993). Tantalum und dessen Salz scheinen auf Grund der genannten Ergebnisse keine relevanten Allergene zu sein und sollten allenfalls gezielt bei entsprechendem Verdacht getestet werden.

\section{Teebaumöl}

Terpinen-4-ol:

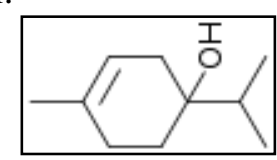

$\alpha$-Terpinen:

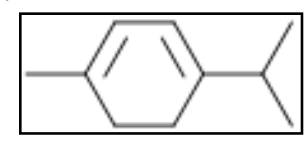

CAS Nr. 562-74-3 (European Commision 2008) CAS Nr. 99-86-5 (European Commision 2008)

$\gamma$-Terpinen:

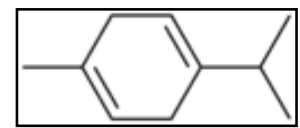

1,8-Cineol:

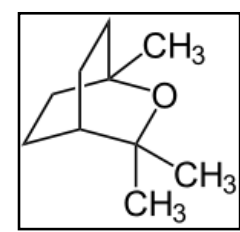

CAS Nr. 99-85-4 (European Commision 2008)

CAS Nr. 470-82-6 (European Commision 2008)

Teebaumöl ist das Destillationsprodukt der nadelartigen Blätter des australischen Teebaumes Melaleuca alternifolia (Pirker et al. 2003). Es ist ein blassgelbes, leicht nach Terpentin riechendes Gemisch bestehend aus über hundert Stoffen: Größtenteils Terpene (Mono- und Sesquiterpene), wobei hier das Terpinen-4-ol (ca. 30-40\%) und die $\alpha$ - (ca. 10\%), und $\gamma$ Terpinen (ca. 20\%) die größten Anteile haben (Pirker, et al. 2003). Weiterer wichtiger Bestandteil ist das 1,8-Cineol (=Eucalyptol) mit einem Anteil von ca. 15\% (Pirker et al. 2003). Australische Ureinwohner benutzten schon Teebaumöl zur Behandlung von Hautkrankheiten, Insektenstichen und Wunden. Heute wird es vermehrt als alternative Behandlungsmethode bei Dermatosen, Akne, Pilz- und Candidainfektionen verwendet (de Groot und Weyland 1992). Mit dem gestiegenen Verbrauch seit den 1980er Jahren wurden auch häufiger Fallberichte über Sensibilisierungen publiziert (Pirker et al. 2003). Da es keine standardisierte Testkonzentration gab, testeten wir zwei Konzentrationen, um die lokale Quote zu erheben. Für Teebaumöl in einer Konzentration von 5\% (DEP.) konnten wir in 0,7\% 
$(\mathrm{n}=1171)$ positive Reaktionen sehen und bei einer Konzentration von 20\% (Oli.) beobachteten wir in 3,7\% ( $\mathrm{n}=1719)$ positive Reaktionen. Im Rahmen einer multizentrischen Studie der DKG, in der unsere Daten vertreten waren, zeigte sich für Teebaumöl in einer Konzentration von 5\% (DEP.) eine Sensibilisierungsquote von 1,1\% ( $n=3375)$ (Pirker et al. 2003). Eine dänische Untersuchung fand eine Quote von $0,5 \%(n=217)$ für eine Konzentration von 10\% (Vas.) und von 0\% ( $\mathrm{n}=160)$ für eine Konzentration von 5\% (Vas.) (Veien et al. 2004). Aus Italien wurde eine Quote von 0,1\% (5\% in Vas., $n=725)$ (Rutherford et al. 2007) berichtet. In Nordamerika wurden in multizentrischen Studien (5\% in Vas.) Quoten von 0,3\% ( $\mathrm{n}=1603)$ (Crawford et al. 2004) und 0,5\% ( $\mathrm{n}=1892)$ publiziert (Warshaw et al. 2009). In Australien zeigte eine multizentrische Untersuchung eine Quote von 1,8\% (10\% in Vas., $n=2320)$ (Rutherford et al. 2007).

Auf Grund der genannten hohen Sensibilisierungsquoten auf Teebaumöl sollten regelmäßige Tests parallel zur Standardreihe durchgeführt werden. Die empfohlene Testkonzentration sollte 5\% (in Oli. oder DEP.) betragen (Pirker et al. 2003). Eine Aufnahme von Teebaumöl in die Standardreihe erscheint nicht sinnvoll, denn zunächst sollte eine StandardTestkonzentration definiert werden, was sich bislang auf Grund der unterschiedlichen Zusammensetzung von Teebaumöl bei der Herstellung als schwierig herausstellt (Pirker et al. 2003). Eine aktuelle Empfehlung deutet auf die Konzentration von 5\% hin (de Groot und Schmidt 2016).

Darüber hinaus spielen Oxidationsvorgänge, bei denen die Einwirkung von Licht und Wärme wesentlich sind, bei der Stabilität eine wichtige Rolle. Es wird angenommen, dass je frischer das Öl, umso geringer das Sensibilisierungspotential ist (Hausen et al. 1999).

\section{Tixocortol-pivalat}

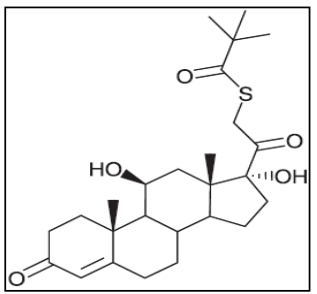

CAS Nr. 55560-96-8 (Le Coz et al. 2006)

Tixocortol-pivalat (TP), Handelsname Pivalone®, ist ein topisches Kortikosteroid zur Anwendung auf die Haut (Kalavala et al. 2007). Die Einteilung der topisch anwendbaren Glukokortikosteroide in Klassen richtet sich entweder nach ihrer Wirkstärke (Niedner 1996) oder nach der hier verwendeten Klassifikation, die chemische und allergologische Gesichtspunkte berücksichtigt. Demnach gibt es 4 allergene Haupt-Gruppen (A-D) (Matura 
und Goossens 2000), wobei das TP zusammen mit Prednisolon oder Hydrokortison in die Gruppe A, d. h. unsubstituierter D-Ring oder C17-Kette, aber C17- und/oder C21-Acetatester, eingeteilt wird. Seit den 1950er Jahren bis heute werden topische Glukokortikosteroide gegen entzündliche und proliferative Hauterkrankungen eingesetzt und haben dabei den Vorteil, dass sie eine relativ geringe systemsiche Wirkung und Toxizität haben (Isaksson 2004). Bei einer Sensibilsierung auf Glukokortikoide besteht die Möglichkeit, dass durch eine Fehlinterpretation das Kontaktekzem maskiert werden kann (Gonul und Gul 2005). Um ein Kontaktekzem zu erfassen, wird empfohlen bestimmte Substanzen als Marker einzusetzen, die repräsentativ Glukokortikoid-Allergien aufdecken können (Isaksson 2004). Zu diesen Markern gehörten TP und Budesonid (Gruppe B), die wir regelmäßig parallel zur Standardreihe testeten. Für TP beobachteten wir in 0,9\% (n=1641) positive Reaktionen in einer Konzentration von $1 \%$ (Vas.). Multizentrische finnische Untersuchungen zeigten Sensibilisierungsquoten von $3,0 \%(n=3416)$ und $3,5 \% \quad(n=10142)$ für TP in $0,1 \%$ (Vas.) (Hasan et al. 2005). Seit dem Jahre 2000 ist TP in der europäischen Standardreihe vertreten (Isaksson et al. 2000), wo für eine Konzentration von 0,1\% (Vas.) eine Quote von 1,3\% $(n=26210)$ gesehen wurde (Bruynzeel et al. 2005). Als Bestandteil der Standardreihe in Grossbritannien zeigte TP eine Quote von 1,5\% (n=3062) mit einer Konzentration von 0,1\% (Vas.) (Britton et al. 2003).

In Studien der NACDG und der Mayo-Clinic in Nordamerika wurden Quoten von 2,7\% $(\mathrm{n}=5807)$ (Marks et al. 2003) und 2,9\% ( $\mathrm{n}=1319)$ (Wetter et al. 2005) für jeweils Konzentrationen von 1\% (Vas.) publiziert. In Australien beschrieb man eine Quote von 0,3\% $(\mathrm{n}=1153)$ für die gleiche Konzentration (1\% in Vas.) (Keegel et al. 2004). Eine der ersten Studien über Kortikoidallergien aus Belgien zeigte eine Quote von 1,1\% (1 \% in Vas., $\mathrm{n}=2073$ ) (Dooms-Goossens und Morren 1992). Eine multizentrische europäische Untersuchung konnte mit jeweils 0,9\% (n=5432) keinen Unterschied der Sensibilisierungsquoten für die Testkonzentrationen von $0,1 \%$ und $1,0 \%$ (jeweils Vas.) beobachten (Isaksson et al. 2000). Diese Ergebnisse wurden von einer multizentrischen britischen Studie bestätigt, wo Testkonzentrationen von $0,1 \%$ und $1 \%$ (beide in Vas.) mit Quoten von $0,7 \%$ und $0,8 \%(n=3747)$ ebenfalls keinen signifikanten Unterscheid ergaben (Kalavala et al. 2007).

Auf Grund der genannten Ergebnisse kann man in Erwägung ziehen, TP als repräsentativer Kortikoid Marker in die Standardreihe aufzunehmen. Die Konzentration sollte dabei 0,1\% (Vas.) betragen, um ein eventuelles Risiko einer Sensibilisierung durch die Testung zu 
minimieren (Goldsmith et al. 1995) und obige Daten belegen, dass dadurch keine niedrigeren Detektionsquoten zu erwarten sind.

\section{Toluolsulfonamid-Formaldehydharz}

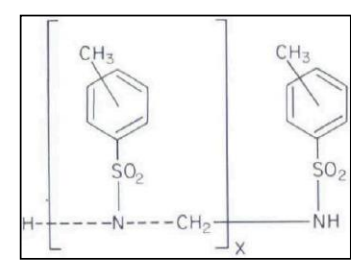

CAS Nr. 25035-71-6 (Liden et al. 1993)

Toluolsulfonamid-Formaldehydharz (toluene sulphonamide formaldehyde resin (TSFR)) wird seit Ende der 1930er Jahre in kosmetischen Nagellacken verwendet, um die Festigkeit und Brüchigkeit von Nägel zu verbessern und das Aufbringen von Farben und Lacken zu erleichtern (Hausen et al. 1995). Weitere Einsatzgebiete sind Laminatharze und industrielle Farblacke. TSFR galt als kaum sensibilisierend, bis Ende der 1980er Jahre in Holland (de Wit et al. 1988) und Mitte der 1990er Jahre in Hamburg in Fallbeispielen Sensibilisierungen beschrieben wurden (Hausen et al. 1995).Weiterhin wurde in einer schwedischen Publikation gefordert, TSFR in die dortige Standardreihe aufzunehmen (Liden et al. 1993). Um die Situation in Göttingen zu klären, wurde TSFR zunächst in einer Konzentration von 10\% (Vas.) untersucht, wo eine Quote von 0,3\% (10\% in Vas., $\mathrm{n}=1018$ ) beobachtet wurde (Fuchs und Gutgesell 1996). Im Verlauf blieb TSFR zunächst in den Tests parallel zur Standardreihe unter Beobachtung und wir fanden in unseren Testungen in $0,2 \% \quad(n=3174)$ positive Reaktionen. Multizentrische finnische Studien zeigten bei unbekannten Testkonzentrationen Quoten von 0,7\% (n=9394) und 0,6\% (n=11788) im Zeitraum zwischen von 1995 bis 2002 (Hasan et al. 2005). Nordamerikanische Studien zeigten für Konzentrationen von 10\% (Vas.) Quoten von 1,4\% ( $\mathrm{n}=1322)$ aus der Mayo-Clinic (Wetter et al. 2005) sowie von 1,5\% $(\mathrm{n}=4097)($ Marks et al. 2000) und 1,3\% (n=5800) (Marks et al. 2003) von der NACDG.

Auf Grund der genannten Ergebnisse erscheinen routinemässige Tests wegen den geringen Quoten nicht sinnvoll. Es sollte bei Verdacht auf eine Nagellackallergie TSFR gezielt untersucht werden.

Nennenswert ist noch, dass Kontaktallergien auf Grund von Nagellackanwendungen mit TSFR bereits im Kindesalter beschrieben sind (Jacob und Stechschulte 2008). 


\section{p-Toluylendiamin}

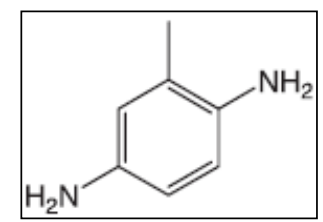

CAS Nr. 95-70-5 (Le Coz et al. 2006)

p-Toluylendiamin (PTD) gehört zu den am häufigsten verwendeten Inhaltsstoffen von Haarfärbemitteln (Winhoven et al. 2007). Es färbt die Haare zu einem dunkleren, rötlichem Ton und ist vor allem in skandinavischen Ländern sehr beliebt. Auch zum Färben von Pelzen wird es eingesetzt (Winhoven et al. 2007). Im Rahmen unserer Tests sollte untersucht werden, ob zusätzlich zu dem standardmäßig getesteten Haarfarbstoffmittel p-Phenylendiamin (PPD) eine Testung mit PTD sinnvoll sein könnte. Wir beoachteten bei einer Konzentration von $1 \%$ (Vas.) in 1,6\% ( $\mathrm{n}=612)$ positive Reaktionen. In einer italienischen Untersuchung wurden für PTD (1\% in Vas.) Sensibilisierungsquoten von 4,6\% $(n=212)$ bei Friseurkunden sowie 13,2\% bei Friseuren $(n=302)$ berichtet (Guerra et al. 1992). In Belgien wurde bei unbekannter Konzentration eine Quote von 1,6\% $(\mathrm{n}=5202)$ bei routinemäßig getesteten Patienten publiziert (Broeckx et al. 1987). Aus England wurde eine Quote von 2,7\% (1\% in Vas., $\mathrm{n}=511$ ) veröffentlicht (Winhoven et al. 2007). In Finnland wurde ein Anstieg der Quoten von 1,4\% $(n=362)$ auf 5,2\% (n=502) im Zeitraum von 1995 bis 2002 bei unbekannter Konzentration beobachtet (Hasan et al. 2005). Zuletzt wurde eine Quote 1,4\% $(\mathrm{n}=8036)$ in einer multizentrischen Studie aus Holland publiziert (Vogel et al. 2017).

Regelmäßige Testungen von PTD scheinen sinnvoll zu sein. Jedoch sollten diese im Rahmen der Friseurstoffreihe und nicht in der Standardreihe durchgeführt werden, da PTD außer in Haarfärbemitteln kaum weitere Bedeutung als Allergen hat (Winhoven et al. 2007). Erwähnenswert ist in diesem Zusammenhang, dass der strukturell verwandte PPD lange Zeit in der Standardreihe vertreten war, bis beobachtet wurde, dass durch Epikutantests aktive Sensibilisierungen stattfanden (Uter et al. 2007a). Deshalb wurde PPD im Jahre 2005 ersatzlos aus der Standardreihe der DKG gestrichen (Geier und Mahler 2016) und nur noch gezielt bei entsprechenden Hinweisen getestet.

\section{Unguentum leniens}

Unguentum leniens (UL), auch als Kühlsalbe bekannt, ist eine Wundsalbe zum Auftragen auf die Haut mit kühlenden Eigenschaften. Die Hauptbestandteile sind Bienenwachs (ca. 7\%), Cetylpalmitat (ca. 8\%), Erdnussöl (ca. 60\%) und destilliertes Wasser (ca. 25\%) (Junghans et al. 2002). Anfang der 1990er Jahre wurde UL in Göttingen zur Behandlung der 
Neurodermitis verabreicht. Im Rahmen einer fortlaufenden Epikutantestung parallel zur Standardreihe erhoben wir die Sensibilisierungsquote und beobachteten dabei in $0,1 \%$ ( $\mathrm{n}=1288$ ) positive Reaktionen. Weitere Publikationen mit Sensibilisierungsquoten liegen nach unserer Kenntnis nicht vor.

Es wird diskutiert, dass Propolis bei einem Kontaktekzem auf UL als Allergen verantwortlich ist (Junghans et al. 2002). Propolis wiederum kann Bestandteil vom Bienenwachs (s.o.) sein und ist als starkes Allergen bekannt (Hausen et al. 1992) und in der Standardreihe vertreten (siehe Propolis Seite 57).

Somit erscheinen Epikutantestungen auf UL allenfalls gezielt anamnestisch sinnvoll, beispielsweise bei bekannter Verwendung von Bienenwachs Produkten.

\section{Zinkdibenzyldithiocarbamat}

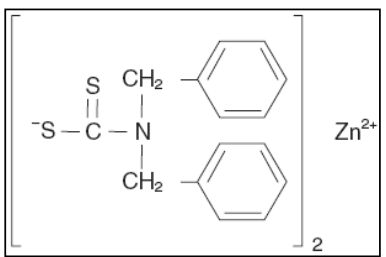

CAS Nr. 14726-36-4 (Geier et al. 2003c)

Zinkdibenzyldithiocarbamat (ZBEC) gehört der Substanzgruppe der Dithiocarbamate an, die als Vulkanisationsbeschleuniger in der Herstellung von Gummihandschuhen eingesetzt werden (Fuchs 1995). Vulkanisation wird definiert als ein Verfahren und Vernetzungsprozess bei dem der vorwiegend plastische Kautschuk durch Verknüpfung von Makromolekülen in den gummielastischen Zustand übergeht (Fuchs 1995). Dithiocarbamate kommen wegen ihren fungiziden Eigenschaften als Konservierungsmittel in verschiedenen industriellen Substanzen vor (Bundesanstalt für Arbeitsschutz und Arbeitsmedizin 1999).

In unseren Tests beobachteten wir für ZBEC in einer Konzentration von 1\% (Vas.) keine positiven Reaktionen ( $\mathrm{n}=554)$, bei wenigen fraglichen Reaktionen. Im Rahmen der multizentrischen Datenauswertung des IVDK zeigte sich eine Sensibilisierungsquote von ebenfalls $0 \%$ (1\% in Vas., $\mathrm{n}=1385$ ) (Geier et al. 2003c). Dies ist uns die bislang einzig bekannte Publikation.

ZBEC scheint im Vergleich zu Zinkdiethyldithiocarbamat (ZDEC), welches als bekanntes Allergen in der Standardserie der DKG vertreten ist (Uter et al. 2016), und Zinkdibutyldithiocarbamat (siehe ZDBC Seite 68) ein deutlich geringeres Sensibilisierungspotential zu haben und regelmäßige Tests erscheinen z. Zt. nicht sinnvoll. 


\section{Zinkdibutyldithiocarbamat}

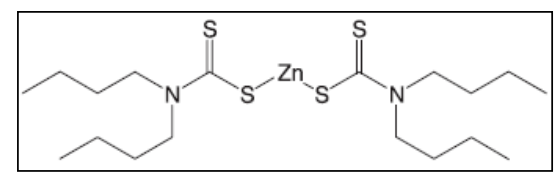

CAS Nr. 136-23-2 (Le Coz et al. 2006)

Zinkdibutyldithiocarbamat (ZDBC) gehört der Substanzgruppe der Dithiocarbamate an, die als Vulkanisationsbeschleuniger in der Herstellung von Gummihandschuhen eingesetzt werden (Fuchs 1995). Vulkanisation wird definiert als Verfahren und Vernetzungsprozess bei dem der vorwiegend plastische Kautschuk durch Verknüpfung von Makromolekülen in den gummielastischen Zustand übergeht (Fuchs 1995). Außerdem kommen sie wegen ihrer fungiziden Eigenschaften als Konservierungsmittel in verschiedenen industriellen Substanzen vor (Bundesanstalt für Arbeitsschutz und Arbeitsmedizin 1999). In unseren Tests konnten wir für ZDBC in einer Konzentration von $1 \%$ (Vas.) in $0,1 \%(n=554)$ positive Reaktionen und in einer Konzentration von 3\% (Vas.) keine positive Reaktion ( $\mathrm{n}=791)$ beobachten. Diese Ergebnisse sind vergleichbar mit Untersuchungen einer multizentrischen Studie der DKG, in der unsere Daten vertreten waren. Die Sensibilisierungsquote von ZDBC betrug dort 0,4\% $(\mathrm{n}=1385)$ in einer Konzentration von 1\% (Vas.) (Geier et al. 2003c).

ZDBC ist Bestandteil vom Carba-Mix (siehe Carba-Mix Seite 32) und wird bei Verdacht auf eine Kontaktallergie auf Gummi im sog. Gummiblock der DKG regelmäßig getestet. Eine Testung darüber hinaus erscheint derzeit nicht sinnvoll. 


\section{Zusammenfassung}

Im Zeitraum von 1996 bis 2004 führten wir Epikutantestungen an 3466 Patienten in der dermatologischen Abteilung der Universitätsklinik in Göttingen durch. Bei diesen Patienten wurde die Indikation zum Epikutantest überwiegend bei Verdacht auf bzw. zum Ausschluss eines allergischen Kontaktekzems gestellt. Der Altersdurchschnitt lag bei 43,5 Jahren mit einem überwiegenden Frauenanteil von ca. $60 \%$.

Es kamen etwa 60 Testsubstanzen zum Einsatz, die parallel zu der Standardreihe in sogenannten Ergänzungsreihen oder auf Grund von individuellem anamnestischen Verdacht getestet wurden.

Im Rahmen dieser Arbeit wurde untersucht, welche Eigenschaften und Verwendungen diese Substanzen besitzen und wie hoch die Sensibilisierungsquoten der einzelnen Substanzen, teils in verschiedenen Konzentrationen und teils in unterschiedlichen Vehikeln, waren. Anschließend wurde ein Literaturvergleich zu nationalen und internationalen Publikationen, insbesondere denen des IVDK (Informationsverbund Dermatologischer Klinken) durchgeführt und Empfehlungen bezüglich weiteren Testungen abgegeben.

Eine Aufnahme in die Standardreihe wird ab einer Sensibilisierungsquote von $>1 \%$ und häufiger Verwendung der Substanz empfohlen.

Insbesondere für folgende Substanzen haben wir wesentliche Ergebnisse in Göttingen beobachtet:

- Dibromidcyanobutan (MDBGN), u. a. ein Konservierungsmittel in Kosmetika: Wir beobachteten hohe Quoten von 2,1\% bzw. 4,9\%. Zusammen mit den Ergebnissen des IVDK hatte es zur Folge, dass MDBGN in die Standardreihe aufgenommen wurde. Durch ähnliche Beobachtungen europaweit wurde diese Substanz schließlich in der EU weitgehend verboten.

- Lyral, ein Duftstoff, da beobachteten wir eine Quote von 3,9\% und im IVDK 1,9\%. Es erfolgte die Aufnahme in Standardreihe.

- Dispersion Blau Mix 124/106 sind Textilfarbstoffe, die durch hohe Quoten sowohl bei uns $(1,6 \%)$ als auch durch den IVDK $(1,1 \%)$ zunächst in die Standardreihe aufgenommen wurden. In der Folgezeit bestätigten sich diese Quoten nicht $(0,6 \%)$ und die Farbstoffe wurden wieder aus dem Standard gestrichen.

- Glutaraldehyd, ein Desinfektionsmittel, zeigte bei uns eine Quote von 0,3\%. Wegen dieses Ergebnisses und den Hinweisen auf ein hohes irritatives Potential konnten wir die international erhobenen Ergebnisse mit Reaktionsquoten bis 5,5\% relativieren. 
- Mercaptobenzothiazol (MBT), ein Vulkanisationsbeschleuniger in der Gummiindustrie, zeigte bei uns zwar eine Quote von 0,8\%, wurde jedoch auf Grund des IVDK-Ergebnisses von 1,8\% in die Standardreihe aufgenommen. Weiterhin liess sich eine optimale Testkonzentration von $2 \%$ (Vas.) definieren.

- Natriumdisulfit, ein Antioxidant u. a. in Arzneimitteln, zeigte bei uns eine hohe Quote mit $1,3 \%$ positiven Reaktionen. Auch internationale Daten weisen darauf hin, dass diese Substanz regelmäßig getestet werden sollte, z. B. im KonservierungsmittelBlock oder in der Standardreihe. Zunächst müssten allerdings die Diskussionen über ein geeignetes Vehikel abgeschlossen werden.

- Propylenglykol, ein flüssiges Lösemittel mit breiter Verwendung in Kosmetika, Medikamenten und Nahrungszusatzstoffen, zeigte in einer Konzentration von 20\% (Aqua) bei uns eine Quote von 2,0\% und im IVDK von 2,3\%. Hiernach scheint eine Aufnahme in die Standardreihe sinnvoll.

- Toluolsulfonamid-Formaldehydharz (TSFR), ein Härter in Nagellacken, zeigte in Göttingen eine Sensibilisierungsquote von 0,2\%. Diese Daten stehen im Gegensatz zu höheren Quoten bis zu 1,5\% in international publizierten Studien. 


\section{Anhang}

Göttingen, 1996 - 2004

$\underline{\text { Reaktionen an Tag } 3 \text { auf DKG-Testreihe Nr. } 1}$

\section{Substanz}

Nickel (II)-sulfat $6 * \mathrm{H} 2 \mathrm{O}$

Duftstoff-Mix

Perubalsam

Dibromdicyanobutan + 2-Phenoxyethanol

Kompositen-Mix

Thiomersal

Kolophonium

Kobalt (II)-chlorid, 6* $\mathrm{H} 2 \mathrm{O}$

Kaliumdichromat

p-Phenylendiamin (Freie Base)(CI 76060)

Dibromdicyanobutan (Methyldibromo Glut.)

Wollwachsalkohole

Quecksilber (II)-amid-chlorid

Dispersions Mix Blau 124/106

Terpentin

$\begin{array}{ccccccccccccc}\text { Konz. } & \text { Ein- } & \text { Vehi- } & \text { Test- } & \text { neg } & ? & \mathrm{f} & + & ++ & +++ & \text { ir } & \text { \%pos } & \text { RI } \\ & \text { heit } & \text { kel } & \text { zahl } & & & & & & & & & \\ 5.00 & \% & \text { Vas } & 2999 & 2447 & 125 & 51 & 156 & 170 & 47 & 3 & 12.4 & 0.4 \\ 8.00 & \% & \text { Vas } & 3115 & 2596 & 263 & 4 & 160 & 72 & 19 & 1 & 8.1 & 0.0 \\ 25.00 & \% & \text { Vas } & 3119 & 2607 & 284 & 4 & 152 & 53 & 16 & 3 & 7.1 & 0.1 \\ 1.00 & \% & \text { Vas } & 650 & 573 & 41 & 0 & 23 & 9 & 4 & 0 & 5.5 & 0.1 \\ 6.00 & \% & \text { Vas } & 1167 & 1037 & 68 & 2 & 31 & 15 & 12 & 2 & 5.0 & 0.1 \\ 0.10 & \% & \text { Vas } & 2593 & 2353 & 112 & 2 & 79 & 37 & 10 & 0 & 4.9 & 0.1 \\ 20.00 & \% & \text { Vas } & 3122 & 2923 & 64 & 3 & 69 & 37 & 26 & 0 & 4.2 & 0.3 \\ 1.00 & \% & \text { Vas } & 3096 & 2768 & 160 & 16 & 72 & 40 & 11 & 29 & 4.0 & 0.3 \\ 0.50 & \% & \text { Vas } & 3114 & 2862 & 124 & 6 & 75 & 32 & 7 & 8 & 3.7 & 0.1 \\ 1.00 & \% & \text { Vas } & 3097 & 2894 & 82 & 8 & 65 & 33 & 14 & 1 & 3.6 & 0.1 \\ 0.30 & \% & \text { Vas } & 846 & 757 & 62 & 1 & 11 & 11 & 4 & 0 & 3.1 & 0.4 \\ 30.00 & \% & \text { Vas } & 3143 & 2894 & 150 & 5 & 68 & 23 & 3 & 0 & 3.0 & 0.2 \\ 1.00 & \% & \text { Vas } & 3146 & 2910 & 139 & 6 & 50 & 32 & 9 & 0 & 2.9 & 0.2 \\ 1.00 & \% & \text { Vas } & 348 & 315 & 21 & 2 & 7 & 3 & 0 & 0 & 2.9 & 0.4 \\ 10.00 & \% & \text { Vas } & 3156 & 2978 & 90 & 1 & 54 & 22 & 11 & 0 & 2.8 & 0.0\end{array}$


Thiuram Mix

(Chlor)-Methylisothiazolinon (MCI/MI)

Dibromdicyanobutan + 2-Phenoxyethanol

Neomycinsulfat

Lyral

Propolis

Bufexamac

Mercaptobenzothiazol

Benzocain (Ethylaminobenzoat)

Epoxidharz

Mercapto-Mix ohne MBT (nur CBS,MBTS,MOR)

Paraben-Mix

N-Isopropyl-N'-phenyl-p-phenylendiamin

Cetylstearylalkohol

Formaldehyd

Zink-diethyldithiosiehemat

p-tert.-Butylphenol-Formaldehydharz

Vaselinum album

$\begin{array}{ccccccccccccc}1.00 & \% & \text { Vas } & 3135 & 3011 & 46 & 1 & 46 & 23 & 8 & 0 & 2.5 & 0.2 \\ 100.00 & \text { ppm } & \text { Aqua } & 3140 & 3019 & 42 & 1 & 54 & 20 & 3 & 1 & 2.5 & 0.3 \\ 0.50 & \% & \text { Vas } & 802 & 771 & 11 & 2 & 7 & 10 & 1 & 0 & 2.2 & 0.2 \\ 20.00 & \% & \text { Vas } & 3124 & 2954 & 97 & 6 & 40 & 21 & 6 & 0 & 2.1 & 0.2 \\ 5.00 & \% & \text { Vas } & 590 & 564 & 13 & 1 & 8 & 4 & 0 & 0 & 2.0 & 0.1 \\ 10.00 & \% & \text { Vas } & 1617 & 1548 & 38 & 0 & 23 & 6 & 2 & 0 & 1.9 & 0.1 \\ 5.00 & \% & \text { Vas } & 1619 & 1574 & 16 & 0 & 17 & 9 & 3 & 0 & 1.8 & 0.3 \\ 2.00 & \% & \text { Vas } & 1164 & 1135 & 13 & 0 & 6 & 8 & 2 & 0 & 1.4 & 0.1 \\ 5.00 & \% & \text { Vas } & 3135 & 3069 & 35 & 4 & 17 & 7 & 3 & 0 & 0.9 & 0.2 \\ 1.00 & \% & \text { Vas } & 3140 & 3075 & 39 & 0 & 11 & 9 & 6 & 0 & 0.8 & 0.2 \\ 1.00 & \% & \text { Vas } & 3142 & 3087 & 27 & 2 & 13 & 12 & 1 & 0 & 0.8 & 0.1 \\ 16.00 & \% & \text { Vas } & 3143 & 2976 & 138 & 1 & 26 & 0 & 0 & 2 & 0.8 & 0.7 \\ 0.10 & \% & \text { Vas } & 3136 & 3065 & 44 & 2 & 15 & 6 & 4 & 0 & 0.8 & 0.3 \\ 20.00 & \% & \text { Vas } & 3144 & 3042 & 78 & 1 & 19 & 3 & 1 & 0 & 0.7 & 0.5 \\ 1.00 & \% & \text { Aqua } & 3134 & 3080 & 29 & 0 & 14 & 6 & 1 & 4 & 0.7 & 0.2 \\ 1.00 & \% & \text { Vas } & 3142 & 3109 & 19 & 0 & 8 & 5 & 1 & 0 & 0.4 & 0.2 \\ 1.00 & \% & \text { Vas } & 3142 & 3092 & 35 & 3 & 7 & 3 & 2 & 0 & 0.4 & 0.5 \\ 100.00 & \% & & 2592 & 2558 & 33 & 1 & 0 & 0 & 0 & 0 & 0.0 & 1.0\end{array}$


$\underline{\text { Reaktionen an Tag } 3 \text { auf Kliniksblock Nr. } 401}$

\begin{tabular}{|c|c|c|c|c|c|c|c|c|c|c|c|c|c|c|c|}
\hline Substanz & Von & Bis & Konz. & $\begin{array}{l}\text { Ein- } \\
\text { heit }\end{array}$ & $\begin{array}{l}\text { Vehi- } \\
\text { kel }\end{array}$ & $\begin{array}{l}\text { Test- } \\
\text { zahl }\end{array}$ & neg & $?$ & $\mathrm{f}$ & + & ++ & +++ & ir & $\%$ pos & $\mathrm{RI}$ \\
\hline Myristylalkohol & 05.10 .04 & 11.03 .05 & 10.00 & $\%$ & Vas & 28 & 21 & 5 & 0 & 2 & 0 & 0 & 0 & 7.1 & -0.4 \\
\hline Dibromdicyanobutan (Methyldibromo & 22.07.99 & 02.11 .04 & 0.30 & $\%$ & Vas & 1102 & 966 & 79 & 3 & 38 & 14 & 2 & 0 & 4.9 & -0.2 \\
\hline \multicolumn{16}{|l|}{ Glut.) } \\
\hline Hg.-Präcipitatsalbe & 23.01 .95 & 09.09 .96 & 1.00 & $\%$ & Vas & 213 & 193 & 11 & 0 & 8 & 0 & 1 & 0 & 4.2 & -0.1 \\
\hline Lyral & 21.06 .01 & 27.12 .01 & 5.00 & $\%$ & Vas & 194 & 186 & 1 & 0 & 5 & 1 & 1 & 0 & 3.6 & 0.8 \\
\hline Carba-Mix & 23.01 .95 & 27.03 .97 & 3.00 & $\%$ & Vas & 388 & 328 & 47 & 0 & 12 & 0 & 0 & 1 & 3.1 & -0.6 \\
\hline Cain-Mix 1 (Procain $1 \%$ und Dibucain & 27.12 .95 & 27.03 .97 & 7.00 & $\%$ & Vas & 611 & 583 & 14 & 0 & 11 & 2 & 1 & 0 & 2.3 & 0.0 \\
\hline \multicolumn{16}{|l|}{$1 \%)$} \\
\hline Dibromdicyanobutan (Methyldibromo & 23.01 .95 & 02.10 .96 & 0.10 & $\%$ & Vas & 612 & 590 & 9 & 0 & 6 & 6 & 1 & 0 & 2.1 & 0.2 \\
\hline \multicolumn{16}{|l|}{ Glut.) } \\
\hline Amalgam (mit Zink) & 09.04 .96 & 27.12 .01 & 5.00 & $\%$ & Vas & 2499 & 2351 & 92 & 5 & 30 & 19 & 2 & 0 & 2.0 & -0.3 \\
\hline Propylenglycol & 23.01 .95 & 27.03 .97 & 20.00 & $\%$ & Aqua & 611 & 547 & 52 & 0 & 11 & 1 & 0 & 0 & 2.0 & -0.6 \\
\hline Propolis & 23.01 .95 & 22.09 .99 & 10.00 & $\%$ & Vas & 1606 & 1549 & 28 & 1 & 16 & 9 & 3 & 0 & 1.7 & -0.0 \\
\hline Kolophonium-Mix II & 16.03 .00 & 06.07 .00 & 15.00 & $\%$ & Vas & 179 & 169 & 6 & 1 & 3 & 0 & 0 & 0 & 1.7 & -0.4 \\
\hline p-Toluylendiamin (freie Base) & 23.01 .95 & 27.03 .97 & 1.00 & $\%$ & Vas & 612 & 588 & 12 & 0 & 7 & 2 & 1 & 2 & 1.6 & -0.2 \\
\hline 1,3-Diphenylguanidin (DPG) & 25.10 .95 & 02.10 .96 & 1.00 & $\%$ & Vas & 385 & 342 & 37 & 0 & 5 & 1 & 0 & 0 & 1.6 & -0.7 \\
\hline Amalgam (ohne Zink) & 23.01 .95 & 02.04 .96 & 5.00 & $\%$ & Vas & 140 & 133 & 5 & 0 & 2 & 0 & 0 & 0 & 1.4 & -0.4 \\
\hline Sesquiterpenlactone Mix & 23.01 .95 & 27.01 .01 & 0.10 & $\%$ & Vas & 2629 & 2566 & 32 & 1 & 12 & 8 & 10 & 0 & 1.1 & -0.0 \\
\hline
\end{tabular}


Tixocortol-pivalat

Mercaptobenzothiazol

p-Aminomethylbenzolsulfonamid

(Mafenid)

Dispers Blau 124

Quaternium 15 (1-(3-Chlorallyl)-3,5,7-..

2-Phenoxyethanol

Imidazolidinylharnstoff (Germall 115)

Poly(hexamethylendiguanid)-HCl

Natriumlaurylsulfat

Glutaraldehyd

Poly(hexamethylendiguanid)- $\mathrm{HCl}$

Hydrocortison-17-butyrat

Propylenglycol

Zink-dibutyldithiocarbamat

Unguentum leniens DAB 10

Natriumlaurylsulfat

Natriumlaurylsulfat

Clobetasol-17-propionat

Zink-dibutyldithiocarbamat

Iodpropinylbutylcarbamat

$\begin{array}{ccccccccccccccc}22.07 .99 & 11.03 .05 & 1.00 & \% & \text { Vas } & 1641 & 1604 & 22 & 0 & 10 & 5 & 0 & 0 & 0.9 & -0.2 \\ 23.01 .95 & 06.07 .00 & 2.00 & \% & \text { Vas } & 2044 & 2013 & 15 & 0 & 6 & 10 & 0 & 0 & 0.8 & 0.0 \\ 23.01 .95 & 27.03 .97 & 10.00 & \% & \text { Vas } & 611 & 604 & 3 & 0 & 3 & 1 & 0 & 0 & 0.7 & 0.1 \\ & & & & & & & & & & & & & & \\ 23.01 .95 & 27.03 .97 & 1.00 & \% & \text { Vas } & 612 & 599 & 9 & 0 & 2 & 0 & 2 & 0 & 0.7 & -0.4 \\ 23.01 .95 & 27.12 .01 & 1.00 & \% & \text { Vas } & 2635 & 2599 & 18 & 1 & 12 & 5 & 0 & 0 & 0.6 & -0.1 \\ 19.02 .02 & 11.03 .05 & 1.00 & \% & \text { Vas } & 554 & 542 & 9 & 0 & 3 & 0 & 0 & 0 & 0.5 & -0.5 \\ 23.01 .95 & 11.03 .05 & 1.00 & \% & \text { Vas } & 3214 & 3175 & 22 & 1 & 14 & 1 & 1 & 0 & 0.5 & -0.2 \\ 02.02 .98 & 22.07 .99 & 2.50 & \% & \text { Aqua } & 651 & 647 & 1 & 0 & 3 & 0 & 0 & 0 & 0.5 & 0.5 \\ 30.12 .99 & 26.08 .04 & 1.00 & \% & \text { Vas } & 1413 & 1199 & 196 & 0 & 6 & 0 & 0 & 12 & 0.4 & -0.9 \\ 23.01 .95 & 16.03 .00 & 0.30 & \% & \text { Vas } & 3218 & 3132 & 21 & 0 & 9 & 0 & 1 & 55 & 0.3 & -0.8 \\ 22.07 .99 & 06.07 .00 & 2.50 & \% & \text { Vas } & 464 & 462 & 0 & 1 & 0 & 1 & 0 & 0 & 0.2 & 0.0 \\ 18.01 .01 & 11.03 .05 & 0.10 & \% & \text { Vas } & 944 & 861 & 81 & 0 & 2 & 0 & 0 & 0 & 0.2 & -1.0 \\ 23.01 .95 & 11.03 .05 & 5.00 & \% & \text { Vas } & 3214 & 3191 & 17 & 0 & 5 & 0 & 0 & 1 & 0.2 & -0.6 \\ 25.10 .95 & 11.03 .05 & 1.00 & \% & \text { Vas } & 3213 & 3192 & 16 & 2 & 2 & 1 & 0 & 0 & 0.1 & -0.7 \\ 08.05 .00 & 11.03 .05 & 100.00 & \% & & 1288 & 1267 & 20 & 0 & 1 & 0 & 0 & 0 & 0.1 & -0.9 \\ 30.12 .99 & 26.08 .04 & 0.25 & \% & \text { Vas } & 1413 & 1373 & 39 & 0 & 1 & 0 & 0 & 0 & 0.1 & -1.0 \\ 30.12 .99 & 26.08 .04 & 0.50 & \% & \text { Vas } & 1413 & 1278 & 128 & 0 & 1 & 0 & 0 & 6 & 0.1 & -1.0 \\ 23.01 .95 & 11.03 .05 & 0.25 & \% & \text { Vas } & 3219 & 2737 & 477 & 1 & 1 & 1 & 0 & 2 & 0.1 & -1.0 \\ 21.06 .01 & 11.03 .05 & 3.00 & \% & \text { Vas } & 791 & 785 & 6 & 0 & 0 & 0 & 0 & 0 & 0.0 & -1.0 \\ 07.05 .01 & 21.06 .01 & 0.10 & \% & \text { Vas } & 44 & 43 & 1 & 0 & 0 & 0 & 0 & 0 & 0.0 & -1.0\end{array}$


Iodpropinylbutylcarbamat

Zinkdibenzyldithiocarbamat

Latex-Handschuhextrakt 4\%ige

Pricklösg.

Incidin plus

$\begin{array}{lllllllllllllll}07.05 .01 & 21.06 .01 & 0.50 & \% & \text { Vas } & 44 & 34 & 10 & 0 & 0 & 0 & 0 & 0 & 0.0 & -1.0 \\ 25.10 .95 & 11.03 .05 & 1.00 & \% & \text { Vas } & 554 & 549 & 5 & 0 & 0 & 0 & 0 & 0 & 0.0 & -1.0 \\ 23.01 .95 & 27.03 .97 & 100.00 & \% & & 178 & 176 & 2 & 0 & 0 & 0 & 0 & 0 & 0.0 & -1.0 \\ 09.09 .96 & 27.03 .97 & 0.10 & \% & \text { Vas } & 263 & 260 & 3 & 0 & 0 & 0 & 0 & 0 & 0.0 & -1.0\end{array}$

$\underline{\text { Reaktionen an Tag } 3 \text { auf Kliniksblock Nr. } 707}$

\begin{tabular}{|c|c|c|c|c|c|c|c|c|c|c|c|c|c|c|}
\hline Substanz & Von & Bis & Konz. & $\begin{array}{l}\text { Ein- } \\
\text { heit }\end{array}$ & $\begin{array}{l}\text { Vehi- } \\
\text { kel }\end{array}$ & $\begin{array}{l}\text { Test- } \\
\text { zahl }\end{array}$ & neg & ? & $\mathrm{f}$ & + & ++ & +++ & ir & $\%$ pos \\
\hline Teebaumöl & 01.04 .98 & 01.04 .98 & 20.00 & $\%$ & Oli & 1719 & 1584 & 71 & 0 & 42 & 17 & 4 & 1 & 3.7 \\
\hline
\end{tabular}

$\underline{\text { Reaktionen an Tag } 3 \text { auf Kliniksblock Nr. } 714}$

\begin{tabular}{|c|c|c|c|c|c|c|c|c|c|c|c|c|c|c|}
\hline Substanz & Von & Bis & Konz. & Ein- & & Test- & neg & $?$ & $=$ & + & ++ & +++ & $r$ & $\%$ pos \\
\hline Teebaumöl & 15.10 .99 & 22.10 .99 & 5.00 & $\%$ & DEP & 1171 & 1156 & 7 & 0 & 5 & 1 & 2 & 0 & 0.7 \\
\hline
\end{tabular}




\section{$\underline{\text { Reaktionen an Tag } 3 \text { auf Kliniksblock Nr. } 713}$}

\begin{tabular}{|c|c|c|c|c|c|c|c|c|c|c|c|c|c|c|c|}
\hline Substanz & Von & Bis & Konz. & $\begin{array}{l}\text { Ein- } \\
\text { heit }\end{array}$ & $\begin{array}{c}\text { Vehi- } \\
\text { kel }\end{array}$ & $\begin{array}{l}\text { Test- } \\
\text { zahl }\end{array}$ & neg & $?$ & $\mathrm{f}$ & + & ++ & +++ & ir & $\%$ pos & RI \\
\hline Bronidox L (5-Brom-5-nitro-1,3-dioxan) & 09.09 .96 & 16.06 .97 & 0.50 & $\%$ & Vas & 212 & 171 & 27 & 1 & 8 & 4 & 0 & 1 & 5.7 & -0.4 \\
\hline Lyral & 07.04 .00 & 06.07 .00 & 5.00 & $\%$ & Vas & 143 & 135 & 2 & 0 & 4 & 2 & 0 & 0 & 4.2 & 0.5 \\
\hline Bufexamac & 06.02 .96 & 26.07.99 & 5.00 & $\%$ & Vas & 1536 & 1482 & 21 & 0 & 7 & 16 & 10 & 0 & 2.1 & 0.2 \\
\hline Dispersions Mix Blau 124/106 & 11.04 .01 & 21.06 .01 & 1.00 & $\%$ & Vas & 62 & 57 & 4 & 0 & 1 & 0 & 0 & 0 & 1.6 & -0.6 \\
\hline Natriumdisulfit & 29.12 .95 & 27.12 .01 & 1.00 & $\%$ & Vas & 2635 & 2553 & 46 & 1 & 26 & 8 & 1 & 0 & 1.3 & -0.1 \\
\hline Dexpanthenol & 03.06 .98 & 27.01 .01 & 5.00 & $\%$ & Vas & 2126 & 2060 & 42 & 2 & 20 & 2 & 0 & 0 & 1.0 & -0.3 \\
\hline 2-Mercaptobenzimidazol & 11.07 .95 & 16.06 .97 & 1.00 & $\%$ & Vas & 231 & 229 & 1 & 0 & 1 & 0 & 0 & 0 & 0.4 & 0.0 \\
\hline Bronidox L (5-Brom-5-nitro-1,3-dioxan) & 12.08 .96 & 27.12 .01 & 0.25 & $\%$ & Vas & 2720 & 2661 & 45 & 0 & 11 & 3 & 0 & 0 & 0.5 & -0.5 \\
\hline 7-Ethylbicyclooxazolidin (Bioban CS & 11.04 .01 & 27.12 .01 & 1.00 & $\%$ & Vas & 256 & 253 & 2 & 0 & 1 & 0 & 0 & 0 & 0.4 & -0.3 \\
\hline \multicolumn{16}{|l|}{ 1246) } \\
\hline Prednicarbat & 11.07 .95 & 16.06 .97 & 0.10 & $\%$ & Vas & 2385 & 2295 & 82 & 1 & 5 & 2 & 0 & 0 & 0.3 & -0.8 \\
\hline Prednicarbat & 11.07 .95 & 11.04 .01 & 0.25 & $\%$ & Vas & 3200 & 3036 & 154 & 1 & 7 & 2 & 0 & 0 & 0.3 & -0.9 \\
\hline Tantalum-V-chloride & 12.11 .96 & 27.12 .01 & 1.00 & $\%$ & Vas & 2783 & 2725 & 50 & 1 & 7 & 0 & 0 & 0 & 0.3 & -0.8 \\
\hline Toluolsulfonamid-Formaldehydharz & 11.07 .95 & 27.12 .01 & 10.00 & $\%$ & Vas & 3174 & 3158 & 9 & 0 & 4 & 3 & 0 & 0 & 0.2 & -0.1 \\
\hline Dimethylformamid & 06.07 .00 & 27.12 .01 & 0.10 & $\%$ & Vas & 1953 & 1936 & 15 & 0 & 2 & 0 & 0 & 0 & 0.1 & -0.8 \\
\hline KKM 702 & 11.07 .95 & 11.04 .01 & 1.00 & $\%$ & Vas & 2379 & 2364 & 14 & 0 & 1 & 0 & 0 & 0 & 0.0 & -0.9 \\
\hline Carboxymethylcellulose (Na-) & 11.07 .95 & 11.04 .01 & 2.00 & $\%$ & Vas & 2385 & 2367 & 18 & 0 & 0 & 0 & 0 & 0 & 0.0 & -1.0 \\
\hline Hexylzimtaldehyd & 21.06 .01 & 27.12 .01 & 2.00 & $\%$ & Vas & 792 & 781 & 11 & 0 & 0 & 0 & 0 & 0 & 0.0 & -1.0 \\
\hline
\end{tabular}


Tantalum

Tantalum

Tantalum-V-chloride
$29.12 .95 \quad 16.6 .97$ $20.02 .96 \quad 12.11 .96 \quad 1.00$

$\begin{array}{llll}12.11 .96 & 11.04 .01 & 0.10 \quad \%\end{array}$

$\begin{array}{ccccccccccc}\text { Vas } & 78 & 78 & 0 & 0 & 0 & 0 & 0 & 0 & 0.0 & . \\ \text { Vas } & 370 & 369 & 1 & 0 & 0 & 0 & 0 & 0 & 0.0 & -1.0 \\ \text { Vas } & 1947 & 1934 & 13 & 0 & 0 & 0 & 0 & 0 & 0.0 & -1.0\end{array}$


Name:

Vorname:

Geburtsdatum:

Ggf. Geburtsname:

Straße:

PLZ:

Ort:

$\begin{array}{ll}\text { Geschlecht: } & \bigcirc \text { weiblich } \\ \bigcirc \text { männlich }\end{array}$

1. Untersuchungsdatum:

\section{ANAMNESE}

\begin{tabular}{|llll|l} 
2.1. Atopische Dermatitis & $\bigcirc$ Ja & $\bigcirc$ Nein & $\bigcirc$ Unbekannt & 3. Atopie-Score: ............ Punkte \\
2.2. Rhinitis allergica & $\bigcirc$ Ja & $\bigcirc$ Nein & $\bigcirc$ Unbekannt & \\
2.3. Allergisches Asthma bronchiale & $\bigcirc$ Ja & $\bigcirc$ Nein & $\bigcirc$ Unbekannt &
\end{tabular}

\subsection{Derzeitiger Beruf}

(bei mehreren der dermatologisch relevante(re)):

4.2. Ausgeübt seit:

Hautveränderungen dabei?

O Ja

Nein

Unklar

\subsection{Ggf. weiterer Beruf}

(falls für die Hauterkrankung relevant):

4.4. Ausgeübt von:

bis:

Hautveränderungen dabei?

Ja $\bigcirc$ Nein $\bigcirc$ Unklar

4.5. In welchen Bereichen werden die Allergene vermutet? (Kontaktstoff-Kategorien)

Baustoffe (Zement, Fliesenkleber ...)

Brille, Hörgerät, Kontaktlinsen u.ä.

Büromaterial

Chemikalien (Sonstige)

Desinfektionsmitte

Farben, Lacke

Farben / frisch gestrichene Räume

Fette, Ole (keine Kühlschmierstoffe)

Friseurstoffe (Dauerwelle, Farbe, Gel ...)

Gummi (Sonstiges)

Handschuhe (Leder, Gummi, Stoff ...)

Hausstäube

Hözer

Hörgerät, Brille, Kontaktlinsen u.ä.

Horgerat, Brile, Kontaktlinsen u.ä.
Implantate, Osteosynthesematerialien (Metall)

Bitte mind. eine Innenraumbeduftung

Kleber

Kleidung, Textilien

Kosmetka, Cremes, Lichtschutzmittel.

Kunststoffe

Kühlschmierstoffe

Laborchemikalien

Leder (Sonstiges, z B. Gürtel, Griffe ...)

Lösemittel, Benzin ..

Med. Hilfsmaterial (Nahtmaterial, EKG-Gel ...)

Medikamente, innerlich

Medikamente, äußerlich

Metalle (Verarbeitung, z.B. Dreher usw.)

Metalle (Sonstiges, z.B. Münzen)

Möbel, neu

Musikinstrumente

Nage kosmetika (Lack, künstliche Nägel ...)

Nahrungsmittel (-zusātze)

Neubauten, neu bezogen

Parfüm, Deo, Rasierwasser.

Pestizide, Herbizide, Insektizide

Pflanzen (keine Nahrungsmittel)

Polituren (Schuhe, Móbel, Auto usw.)

Putz-, Reinigungs-, Waschmittel

Schmuck, Ambanduhr o.ä. (Metall)

Schmuck, Uhren usw. (Nicht-Metall)

Schuhe, Stiefel (Leder, Gummi, Stoff ...)

Seife, Duschgel, Shampoo, Zahncreme..

Sonstiges

Spielzeug, Scherzartike

Tätowierungen

Textilmöbel (Sessel, Teppiche...), neu

Tierhaar, Fele, Peize

Tränengas

Waffen (auch Pyrotechnik)

Weichspüler, Verwendung won

Werkzeug (Metall, Holz, Kunststoff ...

Zahnfullungsmaterialien (Amalgam usw. ...)

Zahnprothesen, -brücken, -spangen

4.6. Besondere Verursachung des Ekzems (in WinAlldat/IVDK unter Anamnese $\rightarrow$ Zusatzfragen)

durch orale Allergenexposition

durch inhalative Allergenexposition

1. Gutachten

2. V. a. Berufsdermatose

V. a. allergisches Kontaktekzem

Ausschluß allerg. Kontaktekzem

5. Berufseingangsuntersuchung
5. Indikation zur Testung

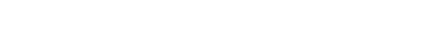


ABSCHLUSSDOKUMENTATION

(Relevanzbeurteilung der Testreaktionen auf gesondertem Bogen)

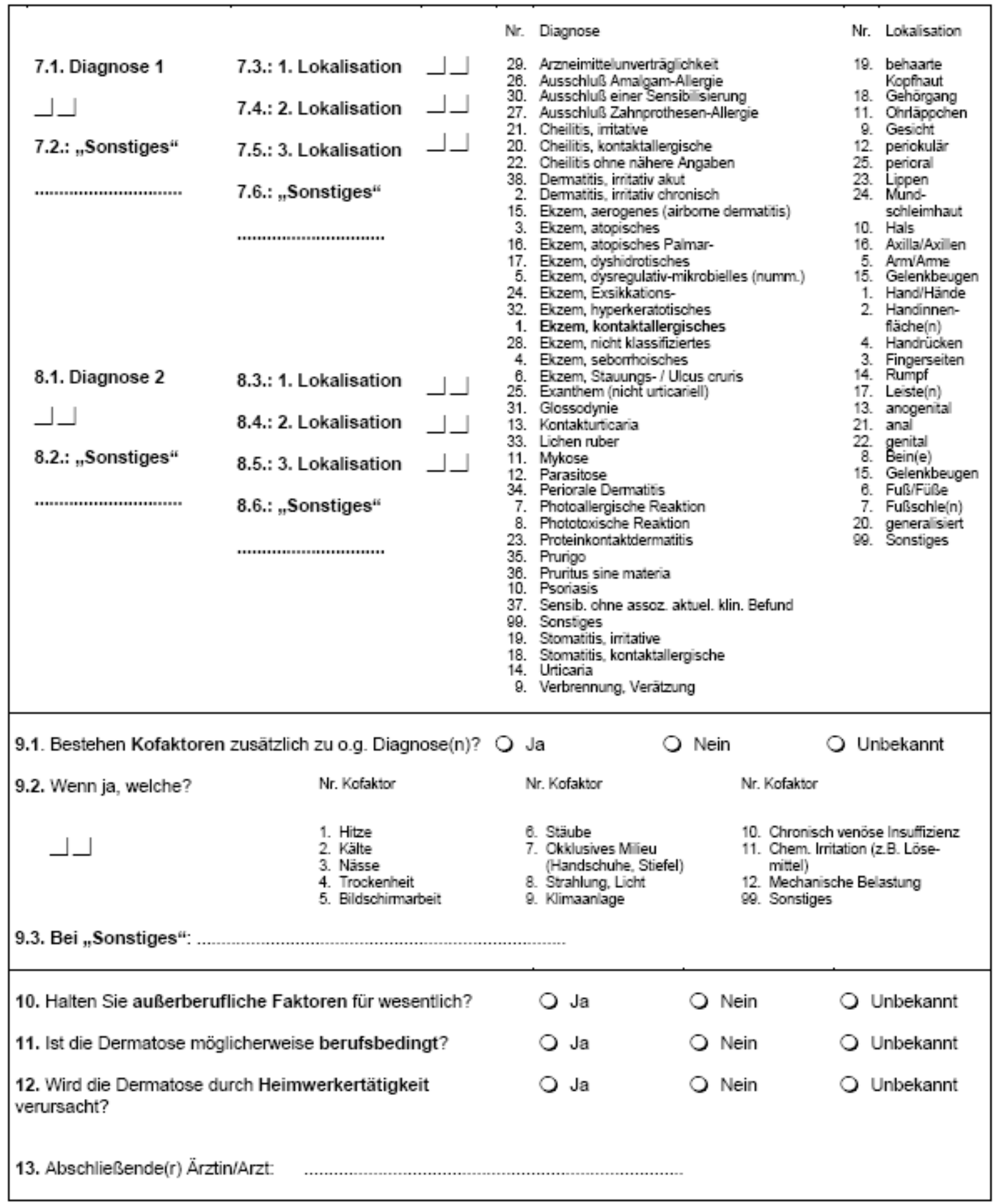


Göttingen, 1996-2004 (3466 Patienten)

$\begin{array}{llll} & & \text { Anzahl } & \text { Prozent } \\ \text { männlich } & \text { M } & 1358 & 39.2 \\ \text { Berufsdermatose } & \text { O } & 340 & 9.8 \\ \text { Atop. Dermatitis } & \text { A } & 687 & 19.8 \\ \text { Hand-Dermatitis } & \text { H } & 933 & 26.9 \\ \text { Bein-Dermatitis } & \text { L } & 268 & 7.7 \\ \text { Face-Dermatitis } & \text { F } & 587 & 16.9 \\ \text { Alter mind. } 40 & \text { A } & 1947 & 56.2\end{array}$

Geschlecht

$\begin{array}{ll}\text { Anzahl } & \text { Prozent } \\ 1358 & 39.2 \\ 2108 & 60.8\end{array}$

Alter

$\begin{array}{ll}\text { Anzahl } & \text { Prozent } \\ 1519 & 43.8 \\ 1947 & 56.2\end{array}$

Alter

$\begin{array}{ll}\text { Anzahl } & \text { Prozent } \\ 120 & 3.5 \\ 239 & 6.9 \\ 537 & 15.5 \\ 620 & 17.9 \\ 545 & 15.7 \\ 563 & 16.2 \\ 465 & 13.4 \\ 277 & 8.0 \\ 86 & 2.5 \\ 11 & 0.3 \\ 3 & 0.1\end{array}$


Göttingen, 1996 (544 Patienten)

Anzahl Prozent

$\begin{array}{llll}\text { männlich } & \text { M } & 200 & 36.8 \\ \text { Berufsdermatose } & \text { O } & 57 & 10.5 \\ \text { Atop. Dermatitis } & \text { A } & 128 & 23.5 \\ \text { Hand-Dermatitis } & \text { H } & 175 & 32.2 \\ \text { Bein-Dermatitis } & \text { L } & 32 & 5.9 \\ \text { Face-Dermatitis } & \text { F } & 94 & 17.3 \\ \text { Alter mind. 40 } & \text { A } & 299 & 55.0\end{array}$

$\begin{array}{lll}\text { Alter } & \text { Anzahl } & \text { Prozent } \\ \text { 00-09 Jahre } & 9 & 1.7 \\ \text { 10-19 Jahre } & 31 & 5.7 \\ \text { 20-29 Jahre } & 112 & 20.6 \\ \text { 30-39 Jahre } & 93 & 17.1 \\ \text { 40-49 Jahre } & 86 & 15.8 \\ \text { 50-59 Jahre } & 87 & 16.0 \\ \text { 60-69 Jahre } & 64 & 11.8 \\ \text { 70-79 Jahre } & 51 & 9.4 \\ \text { 80-89 Jahre } & 10 & 1.8 \\ \text { 90-99 Jahre } & 1 & 0.2 \\ & & \\ \text { Alter } & \text { Anzahl } & \text { Prozent } \\ \text { 00-39 Jahre } & 245 & 45.0 \\ \text { 40-99 Jahre } & 299 & 55.0\end{array}$


Göttingen, 1997 (443 Patienten)

Anzahl Prozent

$\begin{array}{llll}\text { männlich } & \text { M } & 179 & 40.4 \\ \text { Berufsdermatose } & \text { O } & 59 & 13.3 \\ \text { Atop. Dermatitis } & \text { A } & 73 & 16.5 \\ \text { Hand-Dermatitis } & \text { H } & 129 & 29.1 \\ \text { Bein-Dermatitis } & \text { L } & 46 & 10.4 \\ \text { Face-Dermatitis } & \text { F } & 70 & 15.8 \\ \text { Alter mind. 40 } & \text { A } & 257 & 58.0\end{array}$

Alter

00-09 Jahre

10-19 Jahre

20-29 Jahre

30-39 Jahre

40-49 Jahre

50-59 Jahre

60-69 Jahre

70-79 Jahre

80-89 Jahre

90-99 Jahre

Alter

00-39 Jahre

40-99 Jahre
Anzahl Prozent

$\begin{array}{ll}3 & 0.7\end{array}$

$30 \quad 6.8$

$60 \quad 13.5$

$93 \quad 21.0$

$69 \quad 15.6$

$93 \quad 21.0$

$54 \quad 12.2$

$29 \quad 6.5$

$11 \quad 2.5$

$1 \quad 0.2$

Anzahl Prozent

$186 \quad 42.0$

$257 \quad 58.0$ 
Göttingen, 1998 (436 Patienten)

Anzahl Prozent

$\begin{array}{llll}\text { männlich } & \text { M } & 165 & 37.8 \\ \text { Berufsdermatose } & \text { O } & 58 & 13.3 \\ \text { Atop. Dermatitis } & \text { A } & 93 & 21.3 \\ \text { Hand-Dermatitis } & \text { H } & 130 & 29.8 \\ \text { Bein-Dermatitis } & \text { L } & 33 & 7.6 \\ \text { Face-Dermatitis } & \text { F } & 71 & 16.3 \\ \text { Alter mind. 40 } & \text { A } & 231 & 53.0\end{array}$

$\begin{array}{lll}\text { Alter } & \text { Anzahl } & \text { Prozent } \\ \text { 00-09 Jahre } & 2 & 0.5 \\ \text { 10-19 Jahre } & 35 & 8.0 \\ \text { 20-29 Jahre } & 75 & 17.2 \\ \text { 30-39 Jahre } & 93 & 21.3 \\ \text { 40-49 Jahre } & 66 & 15.1 \\ \text { 50-59 Jahre } & 72 & 16.5 \\ \text { 60-69 Jahre } & 60 & 13.8 \\ \text { 70-79 Jahre } & 22 & 5.0 \\ \text { 80-89 Jahre } & 8 & 1.8 \\ \text { 90-99 Jahre } & 3 & 0.7 \\ & & \\ \text { Alter } & \text { Anzahl } & \text { Prozent } \\ \text { 00-39 Jahre } & 205 & 47.0 \\ \text { 40-99 Jahre } & 231 & 53.0\end{array}$


Göttingen, 1999 (480 Patienten)

Anzahl Prozent

$\begin{array}{llll}\text { männlich } & \text { M } & 192 & 40.0\end{array}$

$\begin{array}{llll}\text { Berufsdermatose } & \mathrm{O} & 44 & 9.2\end{array}$

$\begin{array}{llll}\text { Atop. Dermatitis } & \text { A } & 90 & 18.8\end{array}$

Hand-Dermatitis $\quad \mathrm{H} \quad 126 \quad 26.3$

$\begin{array}{llll}\text { Bein-Dermatitis } & \text { L } & 33 & 6.9\end{array}$

$\begin{array}{llll}\text { Face-Dermatitis } & \text { F } & 98 & 20.4\end{array}$

$\begin{array}{llll}\text { Alter mind. } 40 & \text { A } & 275 & 57.3\end{array}$

$\begin{array}{lll}\text { Alter } & \text { Anzahl } & \text { Prozent } \\ \text { 00-09 Jahre } & 7 & 1.5 \\ \text { 10-19 Jahre } & 43 & 9.0 \\ \text { 20-29 Jahre } & 76 & 15.8 \\ \text { 30-39 Jahre } & 79 & 16.5 \\ \text { 40-49 Jahre } & 74 & 15.4 \\ \text { 50-59 Jahre } & 87 & 18.1 \\ \text { 60-69 Jahre } & 69 & 14.4 \\ \text { 70-79 Jahre } & 35 & 7.3 \\ \text { 80-89 Jahre } & 10 & 2.1 \\ & & \\ \text { Alter } & & \\ \text { 00-39 Jahre } & \text { Anzahl } & \text { Prozent } \\ \text { 40-99 Jahre } & 205 & 42.7\end{array}$


Göttingen, $2000 \quad$ (524 Patienten)

Anzahl Prozent

$\begin{array}{llll}\text { männlich } & \text { M } & 217 & 41.4 \\ \text { Berufsdermatose } & \text { O } & 43 & 8.2 \\ \text { Atop. Dermatitis } & \text { A } & 64 & 12.2 \\ \text { Hand-Dermatitis } & \text { H } & 123 & 23.5 \\ \text { Bein-Dermatitis } & \text { L } & 59 & 11.3 \\ \text { Face-Dermatitis } & \text { F } & 94 & 17.9 \\ \text { Alter mind. 40 } & \text { A } & 317 & 60.5\end{array}$

$\begin{array}{lll}\text { Alter } & \text { Anzahl } & \text { Prozent } \\ \text { 00-09 Jahre } & 16 & 3.1 \\ \text { 10-19 Jahre } & 31 & 5.9 \\ \text { 20-29 Jahre } & 72 & 13.7 \\ \text { 30-39 Jahre } & 88 & 16.8 \\ \text { 40-49 Jahre } & 88 & 16.8 \\ \text { 50-59 Jahre } & 87 & 16.6 \\ \text { 60-69 Jahre } & 77 & 14.7 \\ \text { 70-79 Jahre } & 46 & 8.8 \\ \text { 80-89 Jahre } & 17 & 3.2 \\ \text { 90-99 Jahre } & 2 & 0.4 \\ & & \\ \text { Alter } & & \text { Anzahl } \\ \text { 00-39 Jahre } & 207 & 39.5 \\ \text { 40-99 Jahre } & 317 & 60.5\end{array}$


Göttingen, 2001 (433 Patienten)

Anzahl Prozent

$\begin{array}{llll}\text { männlich } & \mathrm{M} & 165 & 38.1\end{array}$

$\begin{array}{llll}\text { Berufsdermatose } & \mathrm{O} & 30 & 6.9\end{array}$

$\begin{array}{llll}\text { Atop. Dermatitis } & \text { A } & 79 & 18.2\end{array}$

$\begin{array}{llll}\text { Hand-Dermatitis } & \mathrm{H} & 83 & 19.2\end{array}$

$\begin{array}{llll}\text { Bein-Dermatitis } & \mathrm{L} & 15 & 3.5\end{array}$

$\begin{array}{llll}\text { Face-Dermatitis } & F & 61 & 14.1\end{array}$

$\begin{array}{llll}\text { Alter mind. } 40 & \text { A } & 225 & 52.0\end{array}$

$\begin{array}{lll}\text { Alter } & \text { Anzahl } & \text { Prozent } \\ \text { 00-09 Jahre } & 33 & 7.6 \\ \text { 10-19 Jahre } & 31 & 7.2 \\ \text { 20-29 Jahre } & 59 & 13.6 \\ \text { 30-39 Jahre } & 82 & 18.9 \\ \text { 40-49 Jahre } & 66 & 15.2 \\ \text { 50-59 Jahre } & 61 & 14.1 \\ \text { 60-69 Jahre } & 57 & 13.2 \\ \text { 70-79 Jahre } & 29 & 6.7 \\ \text { 80-89 Jahre } & 12 & 2.8 \\ \text { Angabe fehlt } & 3 & 0.7 \\ & & \\ \text { Alter } & \text { Anzahl } & \text { Prozent } \\ \text { 00-39 Jahre } & 208 & 48.0 \\ \text { 40-99 Jahre } & 225 & 52.0\end{array}$


Göttingen, 2002 (307 Patienten)

Anzahl Prozent

$\begin{array}{llll}\text { männlich } & \mathrm{M} & 128 & 41.7 \\ \text { Berufsdermatose } & \mathrm{O} & 24 & 7.8 \\ \text { Atop. Dermatitis } & \mathrm{A} & 100 & 32.6 \\ \text { Hand-Dermatitis } & \mathrm{H} & 68 & 22.1 \\ \text { Bein-Dermatitis } & \mathrm{L} & 19 & 6.2 \\ \text { Face-Dermatitis } & \mathrm{F} & 57 & 18.6 \\ \text { Alter mind. 40 } & \mathrm{A} & 147 & 47.9\end{array}$

$\begin{array}{lll}\text { Alter } & \text { Anzahl } & \text { Prozent } \\ \text { 00-09 Jahre } & 38 & 12.4 \\ \text { 10-19 Jahre } & 20 & 6.5 \\ \text { 20-29 Jahre } & 45 & 14.7 \\ \text { 30-39 Jahre } & 57 & 18.6 \\ \text { 40-49 Jahre } & 41 & 13.4 \\ \text { 50-59 Jahre } & 42 & 13.7 \\ \text { 60-69 Jahre } & 33 & 10.7 \\ \text { 70-79 Jahre } & 23 & 7.5 \\ \text { 80-89 Jahre } & 5 & 1.6 \\ \text { 90-99 Jahre } & 3 & 1.0 \\ & & \\ \text { Alter } & & \text { Anzahl } \\ \text { 00-39 Jahre } & 160 & 52.1 \\ \text { 40-99 Jahre } & 147 & 47.9\end{array}$


Göttingen, 2003 (222 Patienten)

$\begin{array}{llll} & & \text { Anzahl } & \text { Prozent } \\ \text { männlich } & \text { M } & 80 & 36.0 \\ \text { Berufsdermatose } & \text { O } & 16 & 7.2 \\ \text { Atop. Dermatitis } & \text { A } & 52 & 23.4 \\ \text { Hand-Dermatitis } & \text { H } & 61 & 27.5 \\ \text { Bein-Dermatitis } & \text { L } & 24 & 10.8 \\ \text { Face-Dermatitis } & \text { F } & 35 & 15.8 \\ \text { Alter mind. 40 } & \text { A } & 135 & 60.8\end{array}$

Alter
00-09 Jahre
10-19 Jahre
20-29 Jahre
30-39 Jahre
40-49 Jahre
50-59 Jahre
60-69 Jahre
70-79 Jahre
80-89 Jahre
90-99 Jahre

Alter

00-39 Jahre

40-99 Jahre

$\begin{array}{ll}\text { Anzahl } & \text { Prozent } \\ 12 & 5.4 \\ 15 & 6.8 \\ 33 & 14.9 \\ 27 & 12.2 \\ 45 & 20.3 \\ 26 & 11.7 \\ 36 & 16.2 \\ 19 & 8.6 \\ 8 & 3.6 \\ 1 & 0.5\end{array}$

$\begin{array}{ll}\text { Anzahl } & \text { Prozent } \\ 87 & 39.2 \\ 135 & 60.8\end{array}$


Göttingen, 2004 (170 Patienten)

Anzahl Prozent

$\begin{array}{llll}\text { männlich } & \mathrm{M} & 73 & 42.9\end{array}$

$\begin{array}{llll}\text { Berufsdermatose } & \mathrm{O} & 22 & 12.9\end{array}$

Atop. Dermatitis $\quad$ A $\quad 31 \quad 18.2$

$\begin{array}{llll}\text { Hand-Dermatitis } & \mathrm{H} & 65 & 38.2\end{array}$

$\begin{array}{llll}\text { Bein-Dermatitis } & \mathrm{L} & 19 & 11.2\end{array}$

Face-Dermatitis $\quad F \quad 24 \quad 14.1$

$\begin{array}{llll}\text { Alter mind. } 40 & \text { A } & 119 & 70.0\end{array}$

$\begin{array}{lll}\text { Alter } & \text { Anzahl } & \text { Prozent } \\ \text { 00-09 Jahre } & 2 & 1.2 \\ \text { 10-19 Jahre } & 7 & 4.1 \\ \text { 20-29 Jahre } & 19 & 11.2 \\ \text { 30-39 Jahre } & 23 & 13.5 \\ \text { 40-49 Jahre } & 30 & 17.6 \\ \text { 50-59 Jahre } & 30 & 17.6 \\ \text { 60-69 Jahre } & 26 & 15.3 \\ \text { 70-79 Jahre } & 27 & 15.9 \\ \text { 80-89 Jahre } & 6 & 3.5 \\ & & \\ \text { Alter } & & \text { Anzahl } \\ \text { 00-39 Jahre } & 51 & 30.0 \\ \text { 40-99 Jahre } & 119 & 70.0\end{array}$




$\begin{array}{lll}\text { Indikation zur Testung } & \text { Anzahl } & \text { Prozent } \\ \text { Ausschluß allergisches Kontaktdermatitis } & 1571 & 45.3 \\ \text { V.a. allergisches Kontaktdermatitis } & 1544 & 44.5 \\ \text { fehlende oder fehlerhafte Angabe } & 105 & 3.0 \\ \text { Gutachten } & 88 & 2.5 \\ \text { V.a. Berufsdermatose } & 73 & 2.1 \\ \text { V.a. Zahnprothesen-Allergie } & 35 & 1.0 \\ \text { Sonstiges } & 23 & 0.7 \\ \text { V.a. Amalgam-Allergie } & 20 & 0.6 \\ \text { Berufseingangsuntersuchung } & 5 & 0.1 \\ \text { V.a. Arzneimittelexanthem } & 2 & 0.1\end{array}$

$\begin{array}{lll}\text { Frühere oder jetzige Atopische Dermatitis? } & \text { Anzahl } & \text { Prozent } \\ \text { Angabe fehlt } & 105 & 3.0 \\ \text { Ja } & 687 & 19.8 \\ \text { Nein } & 2186 & 63.1 \\ \text { unbekannt } & 101 & 2.9\end{array}$

$\begin{array}{lll}\text { Asthma in der Eigenanamnese } & \text { Anzahl } & \text { Prozent } \\ \text { Angabe fehlt } & 117 & 3.4 \\ \text { Ja } & 290 & 8.4 \\ \text { Nein } & 2820 & 81.4 \\ \text { unbekannt } & 239 & 6.9 \\ & & \\ \text { Heuschnupfen in der Eigenanamnese? } & \text { Anzahl } & \text { Prozent } \\ \text { Angabe fehlt } & 114 & 3.3 \\ \text { Ja } & 641 & 18.5 \\ \text { Nein } & 2447 & 70.6 \\ \text { unbekannt } & 264 & 7.6\end{array}$




$\begin{array}{lll}\text { Bestehen zusätzlich Kofaktoren? } & \text { Anzahl } & \text { Prozent } \\ \text { Angabe fehlt } & 112 & 3.2 \\ \text { Ja } & 765 & 22.1 \\ \text { Nein } & 1546 & 44.6 \\ \text { unbekannt } & 1043 & 30.1\end{array}$

$\begin{array}{lll}\text { Zusätzlich bestehende Kofaktoren } & \text { Anzahl } & \text { Prozent } \\ \text { Nässe } & 164 & 21.4 \\ \text { chronisch venöse Insuffizienz } & 132 & 17.3 \\ \text { Okklusives Milieu (Handschuhe o. Stiefel } & 110 & 14.4 \\ \text { Sonstiges } & 109 & 14.2 \\ \text { mechanische Belastung } & 82 & 10.7 \\ \text { Chem. Irritation (z.B. Lösemittel) } & 43 & 5.6 \\ \text { Stäube } & 36 & 4.7 \\ \text { Trockenheit } & 21 & 2.7 \\ \text { Hitze } & 20 & 2.6 \\ \text { Strahlung, Licht } & 20 & 2.6 \\ \text { fehlende oder fehlerhafte Angabe } & 16 & 2.1 \\ \text { Kälte } & 10 & 1.3 \\ \text { Bildschirmarbeit } & 1 & 0.1 \\ \text { Klimaanlage } & 1 & 0.1\end{array}$

$\begin{array}{lll}\text { Sind ausserberufliche Faktoren wesentlich? } & \text { Anzahl } & \text { Prozent } \\ \text { Angabe fehlt } & 149 & 4.3 \\ \text { Ja } & 1889 & 54.5 \\ \text { Nein } & 709 & 20.5 \\ \text { unbekannt } & 719 & 20.7 \\ & & \\ \text { Ist die Dermatose berufsbedingt? } & \text { Anzahl } & \text { Prozent } \\ \text { Angabe fehlt } & 113 & 3.3 \\ \text { Ja } & 340 & 9.8 \\ \text { Nein } & 2684 & 77.4 \\ \text { unbekannt } & 329 & 9.5\end{array}$


In welchen Bereichen werden die Allergene vermutet?

(bis zu drei Nennungen pro Fall möglich)

Kontaktstoff-Kategorie

Anzahl

Prozent

Medikamente, äußerlich

Kosmetika, Cremes, Lichtschutzmittel 850

Seife, Duschgel, Shampoo, Zahncreme... 326

Handschuhe (Leder, Gummi, Stoff...) 232

Parfüm, Deo, Rasierwasser...

Putz-, Reinigungs-, Waschmittel

Zahnfüllungsmaterialien (Amalgam usw...) 185

Zahnprothesen, -brücken, -spangen

Kleidung, Textilien

Desinfektionsmittel

Gummi (sonstiges)

entfällt/keine Angaben

Schuhe, Stiefel (Leder, Gummi, Stoff...)

Nahrungsmittel (-zusätze)

Metalle (sonst., z.B. Münzen)

Metalle (Verarbeitung, z.B. Dreher usw.)

Pflanzen (keine Nahrungsmittel)

Friseurstoffe (Dauerwelle, Farbe, Gel..) 85

Baustoffe (Zement, Fliesenkleber...) $\quad 64$

Kunststoffe

Schmuck, Armbanduhr o.ä. (Metall) $\quad 60$

Med. Hilfsmaterial (Nahtmat.,EKG-Gel...) 60

Farben, Lacke

Leder (sonstiges, z.B. Gürtel, Griffe..) 58

Hölzer

Kühlschmierstoffe

Kleber

Büromaterial

Fette, Öle (keine Kühlschmierstoffe)

Chemikalien (sonstige)

Implantate, Osteosynthesemat. (Metall)

Medikamente, innerlich

Lösungsmittel, Benzin... 


$\begin{array}{lll}\text { Hausstäube } & 34 & 1.0 \\ \text { Werkzeug (Metall, Holz, Kunststoff...) } & 29 & 0.8 \\ \text { Tierhaare, Felle, Pelze } & 25 & 0.7 \\ \text { Sonstiges } & 25 & 0.7 \\ \text { Pestizide, Herbizide, Insektizide... } & 15 & 0.4 \\ \text { Nagelkosmetika (Lack, künstl. Nägel...) } & 10 & 0.3 \\ \text { Konservierungsstoffe (Biozide) } & 4 & 0.1 \\ \text { Schmuck, Uhren usw. (Nicht-Metall) } & 2 & 0.1 \\ \text { Textilmöbel (Sessel, Teppiche..), neu } & 1 & 0.0 \\ \text { Spielzeug, Scherzartikel } & 1 & 0.0 \\ \text { Tätowierungen } & 1 & 0.0\end{array}$


Ekzem, kontaktallergisches

Ekzem, atopisches

Ausschluss einer Sensibilisierung

Dermatitis, irritativ chronisch

Sonstiges

Ekzem, nicht klassifiziertes

Sensib. ohne assoz. aktuel. klin. Befund

Psoriasis

fehlende oder fehlerhafte Angabe

Ausschluss Zahnprothesen-Allergie

Ekzem, dyshidrotisches

Ekzem, Stauungs- / Ulcus cruris

Ausschluss Amalgam-Allergie

entfällt

Ekzem, seborrhoisches

Ekzem, dysregulativ-mikrobielles (numm.) 30

Ekzem, atopisches Palmar-

Ekzem, Exsikkations-

Stomatitis, irritative

Urticaria

Arzneimittelunverträglichkeit

Lichen ruber

Periorale Dermatitis

$11 \quad 0.3$

Pruritus sine materia

$11 \quad 0.3$

Photoallergische Reaktion

10

Mykose

10

0.3

Exanthem (nicht urticariell)

10

0.3

Ekzem, hyperkeratotisches

10

0.3

Stomatitis, kontaktallergische

Prurigo

Ekzem, aerogenes (airborne dermatitis)

Dermatitis, irritativ akut

Phototoxische Reaktion

Glossodynie 


$\begin{array}{lll}\text { Verbrennung, Verätzung } & 1 & 0.0 \\ \text { Kontakturticaria } & 1 & 0.0 \\ \text { Cheilitis, kontaktallergische } & 1 & 0.0 \\ \text { Cheilitis ohne nähere Angaben } & 1 & 0.0\end{array}$

1. Lokalisation zur 1. Abschlussdiagnose Anzahl Prozent

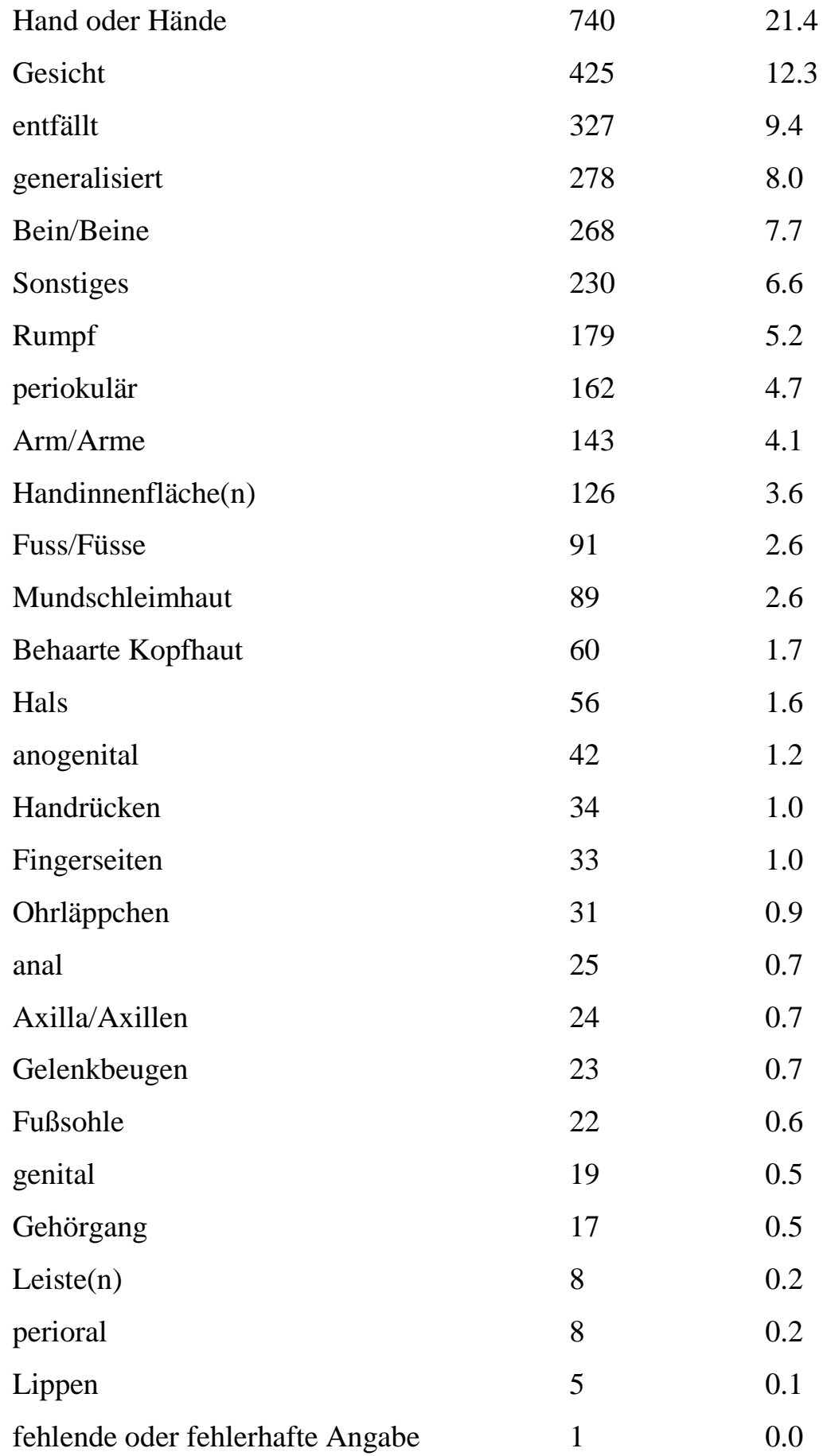


Tätigkeiten mit unbestimmter Exposition

Hausfrauen, Hauswirtschaftliche Betreuer

Übrige Gesundheitsdienstberufe

fehlende oder fehlerhafte Angabe

Sozialpflegerische Berufe, Lehrer, Seelsorger

Mechaniker, Maschinisten, Werkzeugmacher

Ingenieure, Mathematiker, Techniker, Architekten

Raum-, Gebäude-, Fahrzeug-, Straßenreiniger

Berufe des Land-, Wasser- und Luftverkehrs

Köche und Speisenbereiter

Gartenbauer

Verkäufer, Handelsvetreter

Elektriker u.ä.

Tischler, Holzbearbeiter u.ä.

Landwirte, -arbeitskräfte, Weinbauern

Hilfsarbeiter, a.n.g.

Medizinallaboranten, Technische Assistenten u.ä.

Maurer u.ä., Baustoffhersteller

Feinblechner, Installateure, Schlosser

Back- und Konditorwarenhersteller

Sicherheits-, Wach- und Dienstberufe

Zahnärzte

Ärzte

Friseure, Perückenmacher

Gästebetreuer

Chemiker, Chemielaboranten, Chemiearbeiter

Lager-, Transport-, Versandarbeiter, Warenprüfer

Textil- und Bekleidungsberufe

Maler, Lackierer

Wissenschaftler, a.n.g.

Metallverformer (spanend)

Fleisch-, Fischverarbeiter 
Feinmechaniker, Uhrmacher, Edelmetallschmiede

Kassierer

Gummihersteller, Vulkaniseure

Papierhersteller, -verarbeiter, Buchbinder

Kunststoffverarbeiter

Sonstige Körperpfleger

Drucker

Forst-, Jagdberufe

0.2

Glasmacher, Keramiker, Töpfer

0.2

Bauausstatter

0.2

Berufe des Nachrichtenverkehrs

0.2

Strassen-, Tiefbauer

0.1

Apotheker, Apothekenhelfer, Drogisten

0.1

Tierzüchter, -pfleger, Fischereiberufe, Melker

0.1

Metallerzeuger, Walzer, Former, Formgießer

0.1

Ernährungsberufe a.n.g.

0.1

Fotografen, Fotolaboranten

0.1

Tierärzte

0.1

Metalloberflächenbearbeiter, -vergüter, -beschichter

Musikinstrumentenbauer, Puppenmacher, Präparatoren 3

0.1

Lederhersteller, Leder- und Fellverarbeiter

0.1

Raumausstatter, Polsterer

0.1

Künstler, Musiker, Berufssportler

0.1

Wäscher, Chemischreiniger, Bügler

Bergleute, Mineral-, Erdöl-Gewinner, Steinbearbeiter

Metallverformer (spanlos)

$3 \quad 0.1$

Metallverbinder

0.1

Augenoptiker, Refraktionisten

Heilpraktiker

0.1

Schmiede

Getränkehersteller

2

0.1

Tankwarte

1

0.0

Gesundheitssichernde Berufe

0.0 
Liste aller an Tag 3 positiven Substanzen

(jede Substanz nur einmal pro Patient gezählt)

\begin{tabular}{|c|c|c|}
\hline \multirow{2}{*}{\multicolumn{2}{|c|}{$\begin{array}{l}\text { Substanz- } \\
\text { Nummer }\end{array}$}} & \multirow{2}{*}{$\begin{array}{l}\text { Anzahl } \\
\text { Positive }\end{array}$} \\
\hline & & \\
\hline 1946 & Nickel (II)-sulfat $6 * \mathrm{H} 2 \mathrm{O}$ & 411 \\
\hline 1177 & Duftstoff-Mix & 251 \\
\hline 2131 & Perubalsam & 221 \\
\hline 1630 & Kolophonium & 132 \\
\hline 2595 & Thiomersal & 126 \\
\hline 1622 & Kobalt (II)-chlorid, 6*H2O & 123 \\
\hline 1605 & Kaliumdichromat & 114 \\
\hline 2085 & p-Phenylendiamin (Freie Base)(CI 76060) & 112 \\
\hline 2732 & Wollwachsalkohole & 94 \\
\hline 980 & Dibromdicyanobutan (Methyldibromo Glut.) & 92 \\
\hline 2371 & Quecksilber (II)-amid-chlorid & 91 \\
\hline 388 & Amerchol L-101 & 89 \\
\hline 2551 & Terpentin & 87 \\
\hline 2201 & Phenylquecksilberacetat & 86 \\
\hline 5 & (Chlor)-Methylisothiazolinon (MCI/MI) & 77 \\
\hline 2601 & Thiuram Mix & 77 \\
\hline 3114 & Eichenmoos absolue & 70 \\
\hline 1936 & Neomycinsulfat & 67 \\
\hline 604 & Bufexamac & 63 \\
\hline 3051 & Kompositen-Mix (Hausen via IVDK) & 63 \\
\hline 2337 & Propolis & 59 \\
\hline 3002 & Kompositen-Mix & 58 \\
\hline 2302 & Polyvidon-Iod & 55 \\
\hline 3046 & Amalgam (mit Zink) & 55 \\
\hline 1292 & Dibromdicyanobutan + 2-Phenoxyethanol & 51 \\
\hline 3050 & Kolophonium-Mix (Hausen via IVDK) & 51 \\
\hline 2804 & Natriumdisulfit & 50 \\
\hline 2803 & tert.-Butylhydrochinon & 46 \\
\hline 2343 & Propylenglycol & 45 \\
\hline 2008 & Octylgallat & 43 \\
\hline 2103 & Palladiumchlorid & 40 \\
\hline
\end{tabular}


1517 Iod 39

1560 Isoeugenol 35

2796 Tetraethylthiuramdisulfid (Disulfiram) 34

2959 Mutterkrautblüten-Extrakt 34

2960 Sesquiterpenlactone Mix 33

873 Cocamidopropylbetain 32

1732 Mercaptobenzothiazol 32

645 Cain-Mix 1 (Procain 1\% und Dibucain 1\%) 31

3005 Carba-Mix 30

1337 Framycetinsulfat 29

3079 Lyral 29

2609 Tixocortol-pivalat 28

2957 Rainfarnkraut-Extrakt 28

633 Benzocain (Ethylaminobenzoat) 27

2582 Tetramethylthiurammonosulfid 27

951 Dexpanthenol 26

1200 Epoxidharz 26

2050 p-Aminoazobenzol (CI 11000) 26

2107 Paraben-Mix 26

3045 Mercapto-Mix ohne MBT (nur CBS,MBTS,MOR) 26

1172 Dodecylgallat (Laurylgallat) 25

1884 N-Isopropyl-N'-phenyl-p-phenylendiamin 25

2099 p-Toluylendiamin (freie Base) 25

3056 Bronidox L (5-Brom-5-nitro-1,3-dioxan) 25

1496 Hydroxycitronellal 24

1588 Jasminöl 24

1671 Lavendelöl 24

732 Cetylstearylalkohol 23

1335 Formaldehyd 23

1510 Imidazolidinylharnstoff (Germall 115) 23

2789 Dispers Orange 3 (CI 11005) 23

741 Chloracetamid 22

3112 Bronopol (2-Brom-2-nitropropan-1,3-diol) 22

23 Quaternium 15 (1-(3-Chlorallyl)-3,5,7-.. 21

2955 Arnikablüten-Extrakt 21

2470 Sorbinsäure 20

2956 Kamillenblüten-Extrakt 20 
3052 Dispersions Mix Blau 124/106 20

2770 Lemongrasöl 19

2958 Schafgarbenkraut-Extrakt 19

316 Alkylamin-aminobenzoat (Osmaron B) 18

635 Benzoylperoxid 18

2952 Budesonid 18

971 Diazolidinylharnstoff (Germall II) 17

1286 Eugenol 17

1372 Gentamicinsulfat $\quad 17$

1833 Morpholinylmercaptobenzothiazol 17

1874 N-Cyclohexyl-2-benzothiazylsulfenamid 17

2581 Tetramethylthiuramdisulfid 16

636 Benzylalkohol 15

2743 Zimtaldehyd 15

809 Cinchocain- $\mathrm{HCl}$ (Cincain) 14

1109 Dipentamethylen-thiuramdisulfid 14

2644 Triclosan 14

2753 Zink-diethyldithiocarbamat 14

2797 Dibenzothiazyldisulfid (MBTS) 14

2974 Kokosnußdiethanolamid 14

3055 Iodpropinylbutylcarbamat 13

2091 p-tert.-Butylphenol-Formaldehydharz 12

2404 Rosenöl 12

2555 Terpinolen 12

1935 Nelkenöl 11

2954 Lorbeerblätteröl 11

20 1,3-Diphenylguanidin (DPG) 10

507 Benzalkoniumchlorid 10

745 Chloramphenicol 10

1399 Glutaraldehyd 10

1612 Kanamycinsulfat 10

2047 p-Aminomethylbenzolsulfonamid (Mafenid) 10

2746 Zimtalkohol 10

2873 Prednicarbat 10

2949 2-Hydroxyethylmethacrylat (HEMA) 10

362 alpha-Terpinen 9

546 Bergamottöl 9 
629 Bacitracin 8

1142 Dispers Orange 1 (CI 11080) 8

1916 Natriumlaurylsulfat 8

2961 Natriumbenzoat 8

150 4,4'-Diaminodiphenylmethan 7

386 Amcinonid 7

1136 Dispers Blau 124

1269 Ethylenglycol-dimethacrylat $\quad 7$

1495 Hydrocortison-17-butyrat 7

2321 Primin 7

2780 p-Aminophenol (CI 76550) 7

3049 Methylisothiazolinon $\quad 7$

3111 Toluolsulfonamid-Formaldehydharz $\quad 7$

861 Clioquinol (Iodochlorhydroxyquin) 6

1375 Geraniol 6

1873 N-Cyclohexyl-n'-phenyl-p-penylendiamin 6

2474 Sorbitansesquioleat 6

3053 Glyoxal Trimer (Dihydrat) 6

3105 Kolophonium-Mix II 6

103 2-Mercaptobenzimidazol 5

136 3-Aminophenol 5

358 alpha-Phellandren 5

731 Cetylpyridiniumchlorid 5

866 Clotrimazol 5

1807 Methylmethacrylat 5

1851 N,N'-Diphenyl-p-phenylendiamin (DPPD) 5

1933 Natriumthiosulfatoaurat 5

2280 Polyethylenglycolsalbe DAB $8 \quad 5$

2285 Polymyxin B Sulfat 5

2303 Pomeranzenblütenöl 5

2660 Triethylenglycol-dimethacrylat (TEGDMA) 5

2740 Zedernholzöl 5

2806 Triethanolamin (TEA) (Trolamin) 5

2944 Dispers Blau 106

2948 DMDM Hydantoin 5

3033 Hydroxyethylacrylat 5

3110 2-Hydroxypropylmethacrylat (HPMA) 5 
624 Butylhydroxyanisol (BHA) 4

796 Chrom-(III)-chlorid 4

1144 Dispers Rot 1 (CI 11110) 4

1146 Dispers Rot 17 (CI 11210) 4

1150 Dispers Gelb 3 (CI 11855) 4

1636 Kupfer (II)-sulfat, $5 * \mathrm{H} 2 \mathrm{O}$

2276 Poly(hexamethylendiguanid)-HCl 4

2360 Pyrogallol 4

2708 Vanillin 4

2930 Phenol-Formaldehydharz (Resol) 4

2976 1,2-Benzisothiazolin-3-on, Natriumsalz 4

3048 Basis Rot 46

3143 Ylang-ylang (I + II) Öl 4

117 2-Phenoxyethanol 3

630 Benzaldehyd 3

755 Chlorhexidindigluconat 3

1139 Dispers Blau 3 (CI 61505) 3

1188 Eisenchlorid 3

1270 Ethylendiamin-di-HCl 3

1408 Glycerylmonothioglykolat 3

1678 Lidocain-HCl 3

1717 Melamin-Formaldehydharz 3

1828 Monoethanolamin (MEA) 3

2137 Pfefferminzöl 3

2149 Phenol-Formaldehydharz (Novolak) 3

2440 Silbernitrat 3

2633 Triamcinolon-acetonid 3

2751 Zink-dibutyldithiocarbamat 3

2820 p-tert.-Butylcatechin 3

2990 BIS-GMA 3

3030 Dispers Blau 85

3031 Dispers Orange 13

483 Ammoniumpersulfat 2

531 Benzylcinnamat 2

563 Betamethason-17-valerat 2

626 Butylparaben 2

780 Chlorothalonil (TCPN) 2 
862 Clobetasol-17-propionat 2

1120 Diphenylthioharnstoff 2

1156 Dispers Gelb 9 (CI 10375) 2

1298 Farnesol 2

1489 Hydrochinon 2

1493 Hydrocortison 2

1568 Isophorondiamin (IPD) 2

1742 Methenamin (Hexamethylentetramin) 2

1867 N-Butylacrylat 2

2029 Orangenöl 2

2169 Phenylephrin-HCl 2

2187 Phenylglycidylether 2

2323 Procain (p-aminobenzodiethylaminoethanol 2

2564 Tetracain-HCl (Amethocain) 2

2828 Amalgam (ohne Zink) 2

2942 Dispers Blau 1

2981 Ziram 2

3088 Tetramethylol acetylenediurea 2

3093 2-(2-Aminoethoxy)ethanol (Diglykolamin) 2

3145 Narcissus absolut 2

3153 Myristylalkohol 2

360 alpha-Pinen 1

440 Anisöl (Pimpinella Anisum) 1

480 Abietinsäure 1

484 Ammoniumtetrachlor-platinat 1

487 Atranorin 1

665 Campferchinon 1

845 Citronellal 1

1019 Diethylentriamin 1

1110 Dipenten (D,1-Limonen) 1

1184 Edetinsäure-dinatriumsalz 1

1276 Ethylhexyl-acrylat 1

1278 Ethylparaben 1

1290 Eukalyptusöl 1

1437 Harnstoff-Formaldehydharz 1

1450 Hexamethylendiamin (Hexandiamin) 1

1474 Hexylresorcin 1 
1518 Iodoform 1

1520 Iodtinktur 1

1643 L-Carvon 1

1770 Methylheptincarbonat 1

1812 Methylparaben 1

1826 Monobenzon 1

2084 p-Oxybenzoesäurebutylester 1

2346 Propylparaben 1

2385 Resorcin 1

2418 Salicylaldehyd 1

2706 Usninsäure 1

2764 Zinn-II-chlorid 1

2772 Zitronenöl 1

2777 alpha-Amylzimtaldehyd 1

2951 Procain- $\mathrm{HCl}$

2962 Cresylglycidylether 1

2980 Maneb 1

3014 Ciclopiroxolamin 1

3029 Dispers Blau 153

3032 Dispers Braun $1 \quad 1$

3060 Ethoxyquin 1

3062 Butylglycidylether 1

3107 Dexamethasone-21-phosphate disodium salt 1

3115 7-Ethylbicyclooxazolidin (Bioban CS $1246 \quad 1$

3117 (Nitrobutyl)morpholin/.. (Bioban P 1487) 1

3120 Cumarin 1

3121 Citronellol 1

3126 Bisphenol F-Epoxidharz 1

3127 m-Xylidendiamin 1

3142 Baummoos 1

3146 Jasmin absolut 1

3147 Sandelholzöl 1

9004 Metallring $(\mathrm{Zn} / \mathrm{Fe})$, schwarz chromatiert 1

9005 Duftstoff 155428 


\section{Literaturliste}

Aalto-Korte K, Suuronen K, Alanko K (2009): Sodium metabisulfite - a contact allergen? Contact Dermatitis $\underline{60}, 115-117$

Aberer W, Fuchs T, Peters K, Frosch PJ (1993): Propylenglykol: Kutane Nebenwirkungen und Testmethodik. Dermatosen $\underline{41}, 25-27$

Aberer W, Komericki P, Uter W, Hausen BM, Lessmann H, Kränke B, Geier J, Schnuch A (2003): Epidemiologische Überwachung von Kontaktallergenen. Der "Monitorblock" des IVDK. Hautarzt 54, 741-749

Adams RM (1975): Possible substitution for mercaptobenzothiazole in rubber. Contact Dermatitis $\underline{1}, 246$

Affee HS, Dressler DP (1969): Topical application of mafenide acetate. Its association with erythema multiforme and cutaneous reactions. Arch Dermatol 100, 277-281

Agner T, Andersen KE, Bjorkner B, Bruze M, Frosch PJ, Gruvberger B, Hoeck U, Kreilgaard B, Menné T, Sommer J (2001): Standardization of the TRUE Test imidazolidinyl urea and diazolidinyl urea patches. Contact Dermatitis $\underline{45}, 21-25$

Andersen KE, Storrs FJ (1982): Hautreizungen durch Propylenglykol. Hautarzt 33, 12-14

Andersen KE, Benezra C, Burrows D, Camarasa J, Dooms-Goossens A, Ducombs G, Frosch P, Lachapelle JM, Lahti A, Menné T (1987): Contact dermatitis. A review. Contact Dermatitis $\underline{16}, 55-78$

Bandmann HJ (1977): Klinik und Pathologie der allergischen Kontaktdermatitis. Z Hautkr $\underline{52}$, 33-39

Barbaud A, Watson J, Pinault AL, Bursztejn AC, Schmutz JL, Tréchot P (2011): Cutaneous adverse drug reactions caused by delayed sensitization to carboxymethylcellulose. Contact Dermatitis 64, 294-297

Becker D (2013): Allergic contact dermatitis. J Dtsch Dermatol Ges 11, 607-619

Belsito DV (2000): The diagnostic evaluation, treatment, and prevention of allergic contact dermatitis in the new millennium. J Allergy Clin Immunol 105, 409-420

Biro K, Thaci D, Ochsendorf FR, Kaufmann R, Boehncke WH (2003): Efficacy of dexpanthenol in skin protection against irritation: a double-blind, placebo-controlled study. Contact Dermatitis $\underline{49}, 80-84$

Blaschke V, Fuchs T (2003): Relevant allergans by periorbital allergic contact dermatitis. Oxybuprocain, an underestimated allergen. Ophthalmologe 100, 628-632 
Boyvat A, Akyol A, Gurgey E (2005): Contact sensitivity to preservatives in Turkey. Contact Dermatitis $\underline{52}, 329-332$

Brasch J, de Groot AC: Konservierungsmittel. In: Fuchs T, Aberer W (Hrsg.): Kontaktekzem. 2 Auflage; Dustri Verlag Dr. Karl Feistle, München 2007, 535-546

Brasch J, Henseler T, Aberer W, Bäuerle G, Frosch PJ, Fuchs T, Fünfstück V, Kaiser G, Lischka GG, Pilz B (1994): Reproducibility of patch tests. A multicenter study of synchronous left-versus right-sided patch tests by the German Contact Dermatitis Research Group. J Am Acad Dermatol 31, 584-591

Brasch J, Geier J, Henseler T (1995): Evaluation of patch test results by use of the reaction index. An analysis of data recorded by the Information Network of Departments of Dermatology (IVDK). Contact Dermatitis $\underline{33}$, 375-380

Brasch J, Schnuch A, Geier J, Aberer W, Uter W (2004): Iodopropynylbutyl carbamate $0.2 \%$ is suggested for patch testing of patients with eczema possibly related to preservatives. $\mathrm{Br} \mathbf{J}$ Dermatol 151, 608-615

Brehler R, Merk H (2005): In-vitro-Tests zum Nachweis von Kontaktallergien. Hautarzt $\underline{56}$, $1141-1143$

Brinkmeier T, Geier J, Lepoittevin JP, Frosch PJ (2002): Patch test reactions to Biobans in metalworkers are often weak and not reproducible. Contact Dermatitis $\underline{47}, 27-31$

Britton JE, Wilkinson SM, English JS, Gawkrodger DJ, Ormerod AD, Sansom JE, Shaw S, Statham B (2003): The British standard series of contact dermatitis allergens: validation in clinical practice and value for clinical governance. Br J Dermatol 148, 259-264

Broeckx W, Blondeel A, Dooms-Goossens A, Achten G (1987): Cosmetic intolerance. Contact Dermatitis $\underline{16}, 189-194$

Bruchhausen F von (1999): Mafenidacetat. In: v. Bruchhausen F, Ebel S, Hackenthal E, Holzgrabe U (Hrsg.): Hagers Handbuch der Pharmazeutischen Praxis. Stoffe L-Z. Folgeband 5.; Springer Verlag, Berlin Heidelberg 1999, 75

Bruynzeel DP, Maibach HI (1986): Excited skin syndrome (angry back). Arch Dermatol 122, 323-328

Bruynzeel DP, Diepgen TL, Andersen KE, Brandão FM, Bruze M, Frosch PJ, Goossens A, Lahti A, Mahler V, Maibach HI (2005): Monitoring the European standard series in 10 centres 1996-2000. Contact Dermatitis $\underline{53}$, 146-149

Bruze M, Conde-Salazar L, Goossens A, Kanerva L, White IR (1999): Thoughts on sensitizers in a standard patch test series. Contact Dermatitis $\underline{41}, 241-250$

Bruze M, Goossens A, Gruvberger B (2005): Recommendation to include methyldibromo glutaronitrile in the European standard patch test series. Contact Dermatitis $\underline{52}$, 24-28 
Brückner R: Reaktionsmechanismen. Springer Verlag, Berlin Heidelberg 2015

Budde U, Schwanitz H (1991): Kontaktdermatitiden bei Auszubildenden des Frisuerhandwerks in Niedersachsen. Dermatosen in Beruf und Umwelt $\underline{39}, 41-48$

Büchner S (2001): Kontaktdermatitis. Schweiz Med Forum 18, 458-463

Bundesanstalt für Arbeitsschutz und Arbeitsmedizin (1999): Begründung zur Bewertung als sensibilisiernd. Bundesarbeitsblatt $\underline{\mathrm{Juli}}, 1-2$

Cahill J, Nixon R (2005): Allergic contact dermatitis to quaternium 15 in a moisturizing lotion. Australas J Dermatol 뜨, 284-285

Camarasa JG (1987): Contact dermatitis from dimethylformamide. Contact Dermatitis $\underline{16}$, 234

Charles GD, Spencer PJ, Schisler MR, Cifone M, Budinsky RA, Gollapudi BB (2005): Mode of mutagenic action for the biocide Bioban CS-1246 in mouse lymphoma cells and implications for its in vivo mutagenic potential. Toxicol Sci $\underline{84}, 73-80$

Conde-Salazar L, del-Rio E, Guimaraens D, González Domingo A (1993): Type IV allergy to rubber additives: a 10-year study of 686 cases. J Am Acad Dermatol 29, 176-180

Corberan VC, Gomez-Aviles A, Martinez-Gonzalez S, Ivanova S, Domínguez MI, GonzálezPérez ME (2014): Heterogeneous selective oxidation of fatty alcohols: Oxidation of 1tetradecanol as a model substrate. Catal Today $\underline{238}, 49-53$

Cox NH, Mustchin CP (1991): Prolonged spontaneous and alcohol-induced flushing due to the solvent dimethylformamide. Contact Dermatitis $\underline{24}$, 69-70

Crawford GH, Sciacca JR, James WD (2004): Tea tree oil: cutaneous effects of the extracted oil of Melaleuca alternifolia. Dermatitis $\underline{15}, 59-66$

de Groot AC, Weyland JW (1992): Systemic contact dermatitis from tea tree oil. Contact Dermatitis $\underline{27}, 279-280$

de Groot AC, Schmidt E (2016): Tea tree oil: contact allergy and chemical composition. Contact Dermatitis $\underline{75}, 129-143$

de Groot AC, Bruynzeel DP, van Joost T, Weyland JW (1988): Cosmetic allergy from myristyl alcohol. Contact Dermatitis $\underline{19}, 76-77$

de Groot AC, van der Kley AM, Bruynzeel DP, Meinardi MM, Smeenk G, van Joost T, Pavel $S$ (1993): Frequency of false-negative reactions to the fragrance mix. Contact Dermatitis $\underline{28}$, $139-140$ 
de Groot AC, van Ginkel CJ, Weijland JW (1996): Methyldibromoglutaronitrile (Euxyl K 400): an important "new" allergen in cosmetics. J Am Acad Dermatol 35, 743-747

de Groot AC, Geier J, Flyvholm MA, Lensen G, Coenraads PJ (2010): Formaldehydereleasers: Relationship to formaldehyde contact allergy, Part 2: Metalworking fluids and remainder. Contact Dermatitis $\underline{63}$, 129-139

de Wit FS, de Groot AC, Weyland JW, Bos JD (1988): An outbreak of contact dermatitis from toluenesulfonamide formaldehyde resin in a nail hardener. Contact Dermatitis $\underline{18}, 280$ 283

Dickel H, Geier J, Becker D, Fartasch M, Häberle M, Hillen U, John SM, Mahler V, Skudlik C, Weisshaar E (2015): Auswirkung einer berufsbedingten Kontaktallergie gegen Methyldibromoglutaronitril bei der BK 5101. Dermatologie in Beruf und Umwelt $\underline{63}$, 154157

Diepgen TL, Kanerva L (2006): Occupational skin diseases. Eur J Dermatol 16, 324-330

Diepgen TL, Bruynzeel DP, Andersen KE, Brandão FM, Bruze M, Gonçalo M, Goossens A, Lahti A, Mahler V, Menné T (2006): Mercaptobenzothiazole or the mercapto-mix: which should be in the standard series? Contact Dermatitis $\underline{55}$, 36-38

Disch K (1994): Glucoprotamine--a new antimicrobial substance. Zentralbl Hyg Umweltmed $\underline{195}, 357-365$

Dooms-Goossens A, Morren M (1992): Results of routine patch testing with corticosteroid series in 2073 patients. Contact Dermatitis 26 , 182-191

Dooms-Goossens A, Meinardi MM, Bos JD, Degreef H (1994): Contact allergy to corticosteroids: the results of a two-centre study. Br J Dermatol 130, 42-47

Drexler H, Broding HC: Kontaktallergene als Karzinogen. In: Fuchs T, Aberer W (Hrsg.): Kontaktekzem. 2. Auflage; Dustri Verlag Dr. Karl Feistle, München 2007, 781-786

Ducombs G, Benezra C, Talaga P, Andersen KE, Burrows D, Camarasa JG, Dooms-Goossens A, Frosch PJ, Lachapelle JM, Menné T (1990): Patch testing with the "sesquiterpene lactone mix": a marker for contact allergy to Compositae and other sesquiterpene-lactone-containing plants. Contact Dermatitis 22, 249-252

Duggan KC, Walters MJ, Musee J, Harp JM, Kiefer JR, Oates JA, Marnett LJ (2010): Molecular Basis for Cyclooxygenase Inhibition by the Non-steroidal Anti-inflammatory Drug Naproxen. J Biol Chem 285, 34950-34959

Dunkel FG, Elsner P, Burg G (1991): Allergic contact dermatitis from prednicarbate. Contact Dermatitis 24, 59-60 
Ecolab (2017):

https://www.ecolabhealthcare.de/website/seiten/produkte/flaechendesinfektion/konzentrate_al dehydfrei/incidin_plus.php; Zugriff am 25.4.2017

Enk AH, Knop J: Immunologische Grundlagen des allergischen und des irritativen Kontaktekzems. In: Fuchs T, Aberer W (Hrsg.): Kontaktekzem. 2. Auflage; Dustri Verlag Dr. Karl Feistle, München 2007, 13-24

European Commision (2008):

https://ec.europa.eu/health/ph_risk/committees/04_sccp/docs/sccp_o_160.pdf; Zugriff am 25.4.2017

European Medicines Agency (2010):

http://www.ema.europa.eu/ema/index.jsp?curl=pages/news_and_events/news/2010/04/news_ detail_001021.jsp\&mid=WC0b01ac058004d5c1; Zugriff am 25.4.2017

Fernandez JC, Gamboa P, Jauregui I, González G, Antépara I (1992): Concomitant sensitization to enoxolone and mafenide in a topical medicament. Contact Dermatitis 27, 262

Firoz EF, Firoz BF, Williams JF, Henning JS (2007): Allergic contact dermatitis to mafenide acetate: a case series and review of the literature. J Drugs Dermatol $\underline{6}, 825-828$

Fisher AA (1975): Allergic contact dermatitis from Germall 115, a new cosmetic preservative. Contact Dermatitis $\underline{1}, 126$

Foussereau J (1984): History of epicutaneous testing: the blotting-paper and other methods. Contact Dermatitis $\underline{11}, 219-223$

Foussereau J, Menezes-Brandao F, Cavelier C, Herve-Bazin B (1983): Allergy to MBT and its derivatives. Contact Dermatitis $\underline{9}, 514-516$

Frosch PJ, Johansen JD, Menne T, Rastogi SC, Bruze M, Andersen KE, Lepoittevin JP, Giménez Arnau E, Pirker C, Goossens A (1999): Lyral is an important sensitizer in patients sensitive to fragrances. Br J Dermatol $\underline{141}$, 1076-1083

Frosch PJ, Johansen JD, Menne T, Pirker C, Rastogi SC, Andersen KE, Bruze M, Goossens A, Lepoittevin JP, White IR (2002): Further important sensitizers in patients sensitive to fragrances. Contact Dermatitis $\underline{47}, 279-287$

Frosch PJ, Pirker C, Rastogi SC, Andersen KE, Bruze M, Svedman C, Goossens A, White IR, Uter W, Arnau EG (2005): Patch testing with a new fragrance mix detects additional patients sensitive to perfumes and missed by the current fragrance mix. Contact Dermatitis $\underline{52}$, 207215

Fuchs T (1994): Stellungnahme der Deutschen Kontakallergiengruppe zur Amalgam-Allergie. Hautarzt $\underline{45}, 415$ 
Fuchs T: Gummi und Allergie. Dustri Verlag Dr. Karl Feistle, München 1995

Fuchs T: Epikutantest. In: Fuchs T, Aberer W: Kontaktekzem. 2. Auflage; Dustri Verlag Dr. Karl Feistle, München 2007a, 267-326.

Fuchs T: Kontaktekzem durch Gummi. In: Fuchs T, Aberer W: Kontaktekzem. 2. Auflage; Dustri Verlag Dr. Karl Feistle, München 2007b, 567-650.

Fuchs T, Gutgesell C (1996): Is contact allergy to toluene sulphonamide-formaldehyde resin common? Br J Dermatol 135, 1013-1014

Fuchs T, Enders F, Przybilla B (1991): Contact allergy to Euxyl K 400. Dermatosen 39, 151153

Gallo R, Marro I, Sorbara S (2005): Contact allergy from phenoxyethanol in Fitostimoline gauzes. Contact Dermatitis $\underline{53}, 241$

Geier J, Fuchs T (1989): Kontaktallergien durch Bufexamac. Med Klin (Munich) $\underline{84}$, 333-338

Geier J, Gefeller O (1995): Sensitivity of patch tests with rubbermixes: Results of the Information Network of Departments of Dermatology from 1990 to 1993. Am J Contact Dermat $\underline{6}, 143-149$

Geier J, Schnuch A (1995): Glutardialdehyd - Berufsspektrum eines Allergens. Dermatosen $\underline{43}, 30-31$

Geier J, Hausen BM (2000): Epikutantestung mit chemisch modifiziertem Kolophonium. Derm Beruf Umwelt $\underline{48}$, 43-47

Geier J, Hausen BM (2006): Epikutantestung mit chemisch modifiziertem Kolophonium; Teil 2: Untersuchungen mit dem Kolophonium-Mix II. Akt Dermatol 1, 239-242

Geier J, Mahler V (2016): Aktuelles zu den Epikutantestreihen der Deutschen Kontaktallergie-Gruppe. Dermatologie in Beruf und Umwelt 64, 70-75

Geier J, Pilz B, Frosch P, Schnuch A (1994): Contact Allergy due to 2Mercaptobenzimidazole. Dermatosen $\underline{42}, 190-193$

Geier J, Kleinhans D, Peters K (1996a): Kontaktallergien durch industriell verwendete Biozide. Dermatosen 44, 154-159

Geier J, Schnuch A, Fuchs T (1996b): Zunahme der Kontaktallergien gegen Methyldibromoglutaronitril in Deutschland. Allergo J $\underline{19}, 399-402$

Geier J, Lessmann H, Schumacher T, Eckert Ch, Becker D, Boveleth W, Buß M, Eck E, Englitz HG, Koch P (2000a): Vorschlag für die Epikutantestung bei Verdacht auf Kontaktallergie auf Kühlschmierstoffe. Derm Beruf Umwelt $\underline{48}$, 232-236 
Geier J, Schnuch A, Brasch J, Gefeller O (2000b): Patch testing with methyldibromoglutaronitrile. Am J Contact Dermat 11, 207-212

Geier J, Brasch J, Schnuch A, Lessmann H, Pirker C, Frosch PJ (2002a): Lyral has been included in the patch test standard series in Germany. Contact Dermatitis $\underline{46}$, 295-297

Geier J, Uter W, Schnuch A, Brasch J (2002b): Diagnostic screening for contact allergy to mercaptobenzothiazole derivatives. Am J Contact Dermat 13, 66-70

Geier J, Lessmann H, Uter W, Schnuch A (2003a): Occupational rubber glove allergy: results of the Information Network of Departments of Dermatology (IVDK), 1995-2001. Contact Dermatitis $\underline{48}, 39-44$

Geier J, Uter W, Pirker C, Frosch PJ (2003b): Patch testing with the irritant sodium lauryl sulfate (SLS) is useful in interpreting weak reactions to contact allergens as allergic or irritant. Contact Dermatitis $\underline{48}, 99-107$

Geier J, Lessmann H, Rothe A, Uter W, Brasch J, Schnuch A (2003c): Patch testing with zinc dibenzyldithiocarbamate. A multicentre study of the Information Network of Departments of Dermatology and the German Contact Dermatitis Research Group. Contact Dermatitis $\underline{48}$, 209-211

Geier J, Uter W, Lessmann H, Schnuch A (2003d): The positivity ratio--another parameter to assess the diagnostic quality of a patch test preparation. Contact Dermatitis $\underline{48}, 280-282$

Geier J, Lessmann H, Dickel H, Frosch PJ, Koch P, Becker D, Jappe U, Aberer W, Schnuch A, Uter W (2004): Patch test results with the metalworking fluid series of the German Contact Dermatitis Research Group (DKG). Contact Dermatitis 1ㅡ, 118-130

Geier J, Uter W, Schnuch A, Brasch J, Gefeller O (2006a): Both mercaptobenzothiazole and mercapto mix should be part of the standard series. Contact Dermatitis $\underline{55}$, 314-316

Geier J, Lessmann H, Fuchs T, Andersen KE (2006b): Patch testing with myristyl alcohol. Contact Dermatitis $\underline{55}, 366-367$

Geier J, Lessmann H, Mahler V, Pohrt U, Uter W, Schnuch A (2012): Occupational contact allergy caused by rubber gloves - nothing has changed. Contact Dermatitis $\underline{67}, 149-156$

Ghisalberty E (1979): Propolis. Bee World $\underline{60}, 59-84$

Giusti F, Miglietta R, Pepe P, Seidenari S (2004): Sensitization to propolis in 1255 children undergoing patch testing. Contact Dermatitis $\underline{51}, 255-258$

Gloor M: Dermatokortikosteroide (DKST). In: Gloor M, Thoma K, Fluhr J (Hrsg.): Dermatologische Externatherapie. Springer, Berlin Heidelberg 2000, 299-326 
Goldsmith PC, White IR, Rycroft RJ, McFadden JP (1995): Probable active sensitization to tixocortol pivalate. Contact Dermatitis $\underline{33}, 429$

Gonul M, Gul U (2005): Detection of contact hypersensitivity to corticosteroids in allergic contact dermatitis patients who do not respond to topical corticosteroids. Contact Dermatitis $\underline{53}, 67-70$

Goossens A, Matura M, Degreef H (2000): Reactions to corticosteroids: some new aspects regarding cross-sensitivity. Cutis $\underline{65}, 43-45$

Gruvberger B, Bruze M (1997): Contact Allergy to a Commercially Avaliable Preservative Containing 5-Bromo-5-nitro-1,3-dioxan in Humans and Guinea Pigs. Dermatosen 45, 272274

Guerra L, Bardazzi F, Tosti A (1992): Contact dermatitis in hairdressers' clients. Contact Dermatitis 26, 108-111

Guerra L, Tosti A, Bardazzi F, Pigatto P, Lisi P, Santucci B, Valsecchi R, Schena D, Angelini G, Sertoli A (1992): Contact dermatitis in hairdressers: the Italian experience. Gruppo Italiano Ricerca Dermatiti da Contatto e Ambientali. Contact Dermatitis 26, 101-107

Gupta AK, Chow M (2004): Prednicarbate (Dermatop): profile of a corticosteroid. J Cutan Med Surg $\underline{8}, 244-247$

Gutgesell C, Arnold R, Bartels S, Fuchs T (2000): Contact dermatitis to latex is rare. Allergy $\underline{55}, 892-893$

Häberle M, Geier J, Mahler V (2016): Kontaktallergie auf Sulfite: klinische und berufliche Relevanz - Neue Daten aus DKG und IVDK. J Dtsch Dermatol Ges $\underline{14}$, 938-941

Hamada T, Horiguchi S (1978): A case of allergic contact dermatitis due to sodium carboxymethyl cellulose (author's transl). Sangyo Igaku 20, 207-211

Hansen EM, Menne T (1990): Glutaraldehyde: patch test, vehicle and concentration. Contact Dermatitis $\underline{23}, 369-370$

Hasan T, Rantanen T, Alanko K, Harvima RJ, Jolanki R, Kalimo K, Lahti A, Lammintausta K, Lauerma AI, Laukkanen A (2005): Patch test reactions to cosmetic allergens in 1995-1997 and 2000-2002 in Finland--a multicentre study. Contact Dermatitis $\underline{53}$, 40-45

Hausen BM (1993a): The sensitizing potency of Euxyl K 400 and its components 1,2dibromo-2,4-dicyanobutane and 2-phenoxyethanol. Contact Dermatitis $\underline{28}, 149-153$

Hausen BM (1993b): Contact allergy to disperse blue 106 and blue 124 in black "velvet" clothes. Contact Dermatitis $\underline{28}, 169-173$ 
Hausen BM (2005): Evaluation of the main contact allergens in propolis (1995 to 2005). Dermatitis $\underline{16}, 127-129$

Hausen BM, Mohnert J (1989): Contact allergy due to colophony. (V). Patch test results with different types of colophony and modified-colophony products. Contact Dermatitis 20, 295301

Hausen BM, Kuhlwein A, Schulz KH (1982a): Kolophonium Allergie. Ein Beitrag zur Herkunft, Chemie und Verwendung von Kolophonium und Kolophonium-modifizierten Produkten. 1. Derm Beruf Umwelt $\underline{30}$, 107-115

Hausen BM, Kuhlwein A, Schulz KH (1982b): Kolophonium Allergie. Ein Beitrag zur Herkunft, Chemie und Verwendung von Kolophonium und Kolophonium-modifizierten Produkten. 2. Derm Beruf Umwelt 30, 145-152

Hausen BM, Wollenweber E, Senff H, Post B (1987): Propolis allergy. (I). Origin, properties, usage and literature review. Contact Dermatitis $\underline{17}, 163-170$

Hausen BM, Evers P, Stuwe HT, König WA, Wollenweber E (1992): Propolis allergy (IV). Studies with further sensitizers from propolis and constituents common to propolis, poplar buds and balsam of Peru. Contact Dermatitis $\underline{26}, 34-44$

Hausen BM, Milbrodt M, Koenig WA (1995): The allergens of nail polish. Allergenic constituents of common nail polish and toluenesulfonamide-formaldehyde resin (TS-F-R). Contact Dermatitis $\underline{33}, 157-164$

Hausen BM, Reichling J, Harkenthal M (1999): Degradation products of monoterpenes are the sensitizing agents in tea tree oil. Am J Contact Dermat 10, 68-77

Hemmer W, Bracun R, Wolf-Abdolvahab S, Focke M, Götz M, Jarisch R (1997): Maintenance of hand eczema by oral pantothenic acid in a patient sensitized to dexpanthenol. Contact Dermatitis $\underline{37}, 51$

Heratizadeh A, Geier J, Molin S, Werfel T (2017): Contact sensitization in patients with suspected textile allergy. Data of the Information Network of Departments of Dermatology (IVDK) 2007-2014. Contact Dermatitis, epub 24.2.2017

Ippen H (1981): Kontaktallergie auf Dexpanthenol. Derm Beruf Umwelt 29, 45

Isaksson M (2004): Corticosteroids. Dermatol Ther 17, 314-320

Isaksson M, Brandao FM, Bruze M, Goossens A (2000): Recommendation to include budesonide and tixocortol pivalate in the European standard series. ESCD and EECDRG. European Society of Contact Dermatitis. Contact Dermatitis $\underline{43}, 41-42$

Jacob SE, Stechschulte SA (2008): Tosylamide/formaldehyde resin allergy--a consideration in the atopic toddler. Contact Dermatitis $\underline{58}, 312-313$ 
Jacobs MC, White IR, Rycroft RJ, Taub N (1995): Patch testing with preservatives at St John's from 1982 to 1993. Contact Dermatitis $\underline{33}, 247-254$

Jong CT, Statham BN, Green CM, King CM, Gawkrodger DJ, Sansom JE, English JS, Wilkinson SM, Ormerod AD, Chowdhury MM (2007): Contact sensitivity to preservatives in the UK, 2004-2005: results of multicentre study. Contact Dermatitis 57, 165-168

Junghans V, Geier J, Fuchs T (2002): Allergy to propolis caused by beeswax-containing ointment. Am J Contact Dermat $\underline{13}, 87$

Kaaman AC, Boman A, Wrangsjo K, Matura M (2010): Contact allergy to sodium metabisulfite: an occupational problem. Contact Dermatitis $\underline{63}, 110-112$

Kalavala M, Statham BN, Green CM, King C, Ormerod AD, Sansom J, English JS, Wilkinson MS, Horne H, Gawkrodger D (2007): Tixocortol pivalate: what is the right concentration? Contact Dermatitis $\underline{57}, 44-46$

Kanerva L, Estlander T, Alanko K, Jolanki R (2001): Patch test sensitization to Compositae mix, sesquiterpene-lactone mix, Compositae extracts, laurel leaf, Chlorophorin, Mansonone A, and dimethoxydalbergione. Am J Contact Dermat 12, 18-24

Keegel T, Saunders H, Milne R, Sajjachareonpong P, Fletcher A, Nixon R (2004): Topical corticosteroid allergy in an urban Australian centre. Contact Dermatitis $\underline{50}$, 6-14

Kiec-Swierczynska M, Krcisz B (2001): Occupational allergic contact dermatitis in hairdressers due to glutaraldehyde. Contact Dermatitis $\underline{44}$, 185-186

Kimber I, Maibach HI, Msotschi H (2005): Thresholds of contact sensitization from disperse dyes in textiles. Contact Dermatitis $\underline{52}, 295$

King L, Jr., Fransway A, Adkins RB (1993): Chronic urticaria due to surgical clips. N Engl J Med 329, 1583-1584

Koch P (2007): Metalle und Metallsalze. In: Fuchs T, Aberer W (Hrsg.): Kontaktekzem. 2. Auflage; Dustri Verlag Dr. Karl Feistle, München 2007, 481-516

Koo FP, Piletta-Zanin P, Politta-Sanchez S, Milingou M, Saurat JH (2008): Allergic contact dermatitis to carboxymethylcellulose in Comfeel hydrocolloid dressing. Contact Dermatitis $\underline{58}, 375-376$

Korting HC: Dermatotherapie. Springer Verlag, Berlin Heidelberg 1995

Kränke B, Derhaschnig J, Komericki P, Aberer W (1996): Bufexamac is a frequent contact sensitizer. Contact Dermatitis $\underline{34}, 63-64$ 
Lammintausta K, Zimerson E, Winhoven S, Susitaival P, Hasan T, Gruvberger B, Williams J, Beck M, Bruze M (2010): Sensitization to dimethyl fumarate with multiple concurrent patch test reactions. Contact Dermatitis $\underline{62}, 88-96$

Larsen WG (1977): Perfume dermatitis. a study of 20 patients. Arch Dermatol $\underline{113}$, 623-626

Le Coz CJ, Lepoittevin JP: Dictionary of Contact Allergens: Chemical Structures, Sources and References. In: Frosch PJ, Menné T, Lepoittevin J-P (Hrsg.): Contact Dermatitis. 4. Auflage; Springer Verlag, Berlin Heidelberg New York 2006, 943-1105

Lee CH, Maibach HI (1995): The sodium lauryl sulfate model: an overview. Contact Dermatitis $\underline{33}, 1-7$

Lehmann SV, Hoeck U, Breinholdt J, Olsen CE, Kreilgaard B (2006): Characterization and chemistry of imidazolidinyl urea and diazolidinyl urea. Contact Dermatitis $\underline{54}, 50-58$

Lessmann H, Schnuch A, Geier J, Uter W (2005): Skin-sensitizing and irritant properties of propylene glycol. Contact Dermatitis $\underline{53}$, 247-259

Liden C, Berg M, Farm G, Wrangsjö K (1993): Nail varnish allergy with far-reaching consequences. Br J Dermatol $\underline{128}, 57-62$

Lodi A, Ambonati M, Coassini A, Kouhdari Z, Palvarini M, Crosti C (1999): Contact allergy to 'caines' caused by anti-hemorrhoidal ointments. Contact Dermatitis $\underline{41}, 221-222$

Löffler H, Dannenberg S, Effendy I (1998): Der Einfluß des Vehikels auf den Natriumlaurylsulfat-Test. Dermatosen $\underline{46}, 202-205$

Lynde CW, Mitchell JC, Adams RM, Maibach HI, Schorr WJ, Storrs FJ, Taylor J (1982): Patch testing with mercaptobenzothiazole and mercapto-mixes. Contact Dermatitis $\underline{8}, 273-274$

Madan V, Beck MH (2009): Sodium metabisulfite--a contact allergen? Contact Dermatitis $\underline{61}$, 58

Madan V, Walker SL, Beck MH (2007): Sodium metabisulfite allergy is common but is it relevant? Contact Dermatitis $\underline{57}, 173-176$

Maibach HI, Akerson JM, Marzulli FN, Wenninger J, Greif M, Hjorth N, Andersen KE, Wilkinson DS (1980): Test concentrations and vehicles for dermatological testing of cosmetic ingredients. Contact Dermatitis $\underline{6}, 369-404$

Marks JG Jr., Belsito DV, DeLeo VA, Fowler JF Jr., Fransway AF, Maibach HI, Mathias CG, Pratt MD, Rietschel RL, Sherertz EF (2000): North American Contact Dermatitis Group patch-test results, 1996-1998. Arch Dermatol 136, 272-273 
Marks JG Jr., Belsito DV, DeLeo VA, Fowler JF Jr., Fransway AF, Maibach HI, Mathias CG, Pratt MD, Rietschel RL, Sherertz EF (2003): North American Contact Dermatitis Group patch-test results, 1998 to 2000. Am J Contact Dermat 14, 59-62

Matissek R (1983): Zur Analytik von 5-Brom-5-nitro-1,3-dioxan (Bronidox) - einer antimikrobiell wirksamen Substanz für Cosmetica. Zeitschrift für LebensmittelUntersuchung und Forschung $\underline{177,271-277}$

Matura M, Goossens A (2000): Contact allergy to corticosteroids. Allergy $\underline{55}$, 698-704

Maurer S, Seubert A, Seubert S, Fuchs T (1995): Kontaktallergie auf Textilien. Dermatosen $\underline{43}, 63-68$

von Mayenburg J, Frosch P, Fuchs T, Aberer W, Bäurle G, Brehler R, Busch R, Gaber G, Hensel O, Koch P (1996): Quecksilber und Amalgam. Dermatosen 44, 213-221

Mintrop B: Quecksilberallergie - Beitrag zur Problematik der Testung mit Quecksilberverbindungen. Med. Diss. Göttingen 1994

Montoro J, Valero A, Elices A, Rubira N, Serra-Baldrich E, Amat P, Malet A (2000): Anaphylactic shock after intra-articular injection of carboxymethylcellulose. Allergol Immunopathol (Madr) 28 , 332-333

Muroi N, Mori S, Ono S, Takahashi HK, Fujii T, Hosoi S, Henmi K, Nishibori M (2002): Allergy to carboxymethylcellulose. Allergy $\underline{57}, 1212-1213$

Natkunarajah J, Osborne V, Holden C (2008): Allergic contact dermatitis to iodopropynyl butylcarbamate found in a cosmetic cleansing wipe. Contact Dermatitis $\underline{58}, 316-317$

Nettis E, Colanardi MC, Soccio AL, Ferrannini A, Tursi A (2002): Occupational irritant and allergic contact dermatitis among healthcare workers. Contact Dermatitis $\underline{46}, 101-107$

Niedner R (1996): Glukokortikosteroide in der Dermatologie. Dt Ärztebl 93, 2868-2872

Oliphant T, Mitra A, Wilkinson M (2012): Contact allergy to sodium sulfite and its relationship to sodium metabisulfite. Contact Dermatitis $\underline{66}, 128-130$

Paulsen E (1992): Compositae dermatitis: a survey. Contact Dermatitis $\underline{26}$, 76-86

Paulsen E, Andersen KE, Hausen BM (2001): An 8-year experience with routine SL mix patch testing supplemented with Compositae mix in Denmark. Contact Dermatitis $\underline{45}, 29-35$

Perrenoud D, Bircher A, Hunziker T, Suter H, Bruckner-Tuderman L, Stäger J, Thürlimann W, Schmid P, Suard A, Hunziker N (1994): Frequency of sensitization to 13 common preservatives in Switzerland. Swiss Contact Dermatitis Research Group. Contact Dermatitis $\underline{30}, 276-279$ 
Perret CM, Happle R (1989): Contact allergy to bufexamac. Contact Dermatitis $\underline{20}$, 307-308

Pirker C, Hausen BM, Uter W, Hillen U, Brasch J, Bayerl C, Lippert U, Fuchs T, Aberer W, Fartasch M (2003): Sensibilisierung auf Teebaumöl in Deutschland und Osterreich. Eine multizentrische Studie der Deutschen Kontaktallergiegruppe. J Dtsch Dermatol Ges 1, 629634

Piskin G, Meijs MM, van der Ham R, Bos JD (2006): Glove allergy due to 1,3diphenylguanidine. Contact Dermatitis $\underline{54}, 61-62$

Potokar M, Greb W, Ippen H, Maibach HI, Schulz KH, Lorenz P, Gloxhuber Ch (1976): Bronidox, ein neues Konservierungsmittel für die Kosmetik. Fette, Seifen- und Anstrichmittel $\underline{78}, 269-276$

Pratt MD, Mufti A, Lipson J, Warshaw EM, Maibach HI, Taylor JS, Sasseville D, DeKoven JG, Zirwas MJ, Fransway AF (2017): Patch Test Reactions to Corticosteroids: Retrospective Analysis From the North American Contact Dermatitis Group 2007-2014. Dermatitis 288, 5863

Przybilla B, Ring J (1987): Sulfit-Überempfindlichkeit. Hautarzt $\underline{38}$, 445-448

Rastogi SC, Johansen JD, Frosch P, Menné T, Bruze M, Lepoittevin JP, Dreier B, Andersen KE, White IR (1998): Deodorants on the European market: quantitative chemical analysis of 21 fragrances. Contact Dermatitis $\underline{38}, 29-35$

Riemersma WA, Schuttelaar ML, Coenraads PJ (2004): Type IV hypersensitivity to sodium metabisulfite in local anaesthetic. Contact Dermatitis $\underline{51}, 148$

Rietschel RL, Warshaw EM, Sasseville D, Fowler JF Jr., DeLeo VA, Belsito DV, Taylor JS, Storrs FJ, Mathias CG, Maibach HI (2007): Sensitivity of petrolatum and aqueous vehicles for detecting allergy to imidazolidinylurea, diazolidinylurea, and DMDM hydantoin: a retrospective analysis from the North American Contact Dermatitis Group. Dermatitis 18, $155-162$

Romaguera C, Vilaplana J (1995): Contact dermatitis from tantalum. Contact Dermatitis $\underline{32}$, 184

Rutherford T, Nixon R, Tam M, Tate B (2007): Allergy to tea tree oil: retrospective review of 41 cases with positive patch tests over 4.5 years. Australas J Dermatol $\underline{48}, 83-87$

Sakemi K, Ito R, Umemura T, Ohno Y, Tsuda M (2002): Comparative toxicokinetic/toxicodynamic study of rubber antioxidants, 2-mercaptobenzimidazole and its methyl substituted derivatives, by repeated oral administration in rats. Arch Toxicol $\underline{76}, 682-$ 691

Sanz de Galdeano C, Aguirre A, Oleaga JM, Goday J, Diaz Perez JL (1993): Allergic contact dermatitis from topical mafenide. Contact Dermatitis $\underline{28}, 249$ 
Schäfer-Korting M, Korting HC, Kerscher MJ, Lenhard S (1993): Prednicarbate activity and benefit/risk ratio in relation to other topical glucocorticoids. Clin Pharmacol Ther $\underline{54}$, 448-456

Schnuch A, Geier J, Uter W, Frosch PJ (1998): Patch testing with preservatives, antimicrobials and industrial biocides. Results from a multicentre study. Br J Dermatol $\underline{138}$, 467-476

Schnuch A, Geier J, Brasch J, Fuchs T, Pirker C, Schulze-Dirks A, Basketter DA (2000): Polyhexamethylenebiguanide: a relevant contact allergen? Contact Dermatitis $\underline{42}, 302-303$

Schnuch A, Aberer W, Agathos M, Brasch J, Frosch PJ, Fuchs T, Richter G (2001): Leitlinien der Deutschen Dermatologischen Gesellschaft (DDG) zur Durchführung des Epikutantests mit Kontaktallergenen. Hautarzt 22, 864-866

Schnuch A, Geier J, Brasch J, Uter W (2002a): The preservative iodopropynyl butylcarbamate: frequency of allergic reactions and diagnostic considerations. Contact Dermatitis $\underline{46}, 153-156$

Schnuch A, Uter W, Geier J, Gefeller O (2002b): Epidemiology of contact allergy: an estimation of morbidity employing the clinical epidemiology and drug-utilization research (CE-DUR) approach. Contact Dermatitis $\underline{47}, 32-39$

Schnuch A, Gefeller O, Uter W (2005a): Eine heimtückische und häufige Nebenwirkung: Kontaktallergien durch das Ekzemtherapeutikum Bufexamac. Ergebnisse des IVDK. Dtsch Med Wochenschr 130, 2881-2886

Schnuch A, Kelterer D, Bauer A, Schuster Ch, Aberer W, Mahler V, Katzer K, Rakoski J, Jappe U, Krautheim A (2005b): Quantitative patch and repeated open application testing in methyldibromo glutaronitrile-sensitive patients. Contact Dermatitis $\underline{52}$, 197-206

Schnuch A, Uter W, Geier J, Brasch J, Frosch PJ (2005c): Überwachung der Kontaktallergie: zur „Wächterfunktion“ des IVDK. Allergo J 14, 618-629

Schnuch A, Geier J, Uter W, Basketter DA, Jowsey IR (2007a): The biocide polyhexamethylene biguanide remains an uncommon contact allergen. Contact Dermatitis $\underline{56}$, 235-239

Schnuch A, Uter W, Geier J, Lessmann H, Frosch PJ (2007b): Sensitization to 26 fragrances to be labelled according to current European regulation. Results of the IVDK and review of the literature. Contact Dermatitis $\underline{57}, 1-10$

Schnuch A, Aberer W, Agathos M, Becker D, Brasch J, Elsner P, Frosch PJ, Fuchs T, Geier J, Hillen U (2008): Performing patch testing with contact allergens. J Dtsch Dermatol Ges $\underline{6}$, 770-775

Schnuch A, Geier J, Lessmann H, Arnold R, Uter W (2012): Surveillance of contact allergies: methods and results of the Information Network of Departments of Dermatology (IVDK). Allergy $\underline{67}, 847-857$ 
Scholz A: "Von den Schädlichkeiten der Haut" - Historische Aspekte des Kontaktekzmes. In: Fuchs T, Aberer W (Hrsg.): Kontaktekzem. 2. Auflage; Dustri Verlag Dr. Karl Feistle, München 2007, 1-12

Schülke (2006): https://www.schuelke.com/ch-de/products/euxyl-K-702.php; Zugriff am 25.4.2017

Shaffer MP, Belsito DV (2000): Allergic contact dermatitis from glutaraldehyde in healthcare workers. Contact Dermatitis $\underline{43}, 150-156$

Sherertz E, Swartz S (1994): Careful With Carba Mix. Am J Contact Dermat $\underline{5}$, 44-45

Skudlik C, Schnuch A, Uter W, Schwanitz HJ (2002): Berufsbedingtes Kontaktekzem nach Anwendung einer Dexpanthenol-haltigen Salbe und Überblick über die IVDK-Daten zu Dexpanthenol. Akt Dermatol 28, 398-401

Sommer S, Wilkinson SM, English JS, Gawkrodger DJ, Green C, King CM, Powell S, Sansom JE, Shaw S (2002): Type-IV hypersensitivity to betamethasone valerate and clobetasol propionate: results of a multicentre study. Br J Dermatol 147, 266-269

Stables GI, Wilkinson SM (1998): Allergic contact dermatitis due to panthenol. Contact Dermatitis $\underline{38}, 236-237$

Steber J, Schröder FR (1996): Glucoprotamin ${ }^{\circledR}$ - ein antimikrobieller Wikrstoff mit günstigen Abbaueigenschaften. Hyg Med 22, 19-26

Steigleder G: Dermatologie and Venerologie. 5. Auflage; Georg Thieme Verlag, Stuttgart 1987

Stingeni L, Lisi P (1999): Contact allergy to prednicarbate: frequency of positive reactions in consecutively-patch-tested patients. Contact Dermatitis $\underline{40}$, 286-287

Susitaival P, Winhoven SM, Williams J, Lammintausta K, Hasan T, Beck MH, Gruvberger B, Zimerson E, Bruze M (2010): An outbreak of fur niture related dermatitis ('sofa dermatitis') in Finland and the UK: history and clinical cases. J Eur Acad Dermatol Venereol 24, 486-489

Thoma K: Dermatische Grundlagen und ihre therapeutischen Funtionen. In: Gloor M, Thoma K, Fluhr J (Hrsg.): Dermatologische Externatherapie. Springer, Berlin Heidelberg 2000, 27 58

Thomson KF, Wilkinson SM, Powell S, Beck MH (1999): The prevalence of corticosteroid allergy in two U.K. centres: prescribing implications. Br J Dermatol 141, 863-866

Tosti A, Piraccini BM, Bardazzi F (1990): Occupational contact dermatitis due to quaternium 15. Contact Dermatitis $\underline{23}, 41-42$

Tosti A, Vincenzi C, Trevisi P, Guerra L (1995): Euxyl K 400: incidence of sensitization, patch test concentration and vehicle. Contact Dermatitis $\underline{33}$, 193-195 
Tosti A, Vincenzi C, Guerra L, Andrisano E (1996): Contact dermatitis from fatty alcohols. Contact Dermatitis $\underline{35}, 287-289$

Tupker RA, Willis C, Berardesca E, Lee CH, Fartasch M, Agner T, Serup J (1997): Guidelines on sodium lauryl sulfate (SLS) exposure tests. A report from the Standardization Group of the European Society of Contact Dermatitis. Contact Dermatitis 37, 53-69

Uter W, Geier J, Schnuch A (1999): The MOAHLFA index in 17 centers of the Information Network of Departments of Dermatology (IVDK) over 6 years. Contact Dermatitis $\underline{41}, 343$ 344

Uter W, Balzer C, Geier J, Frosch PJ, Schnuch A (2005): Patch testing with patients' own cosmetics and toiletries- results of the IVDK*, 1998-2002. Contact Dermatitis $\underline{53}$, 226-233

Uter W, Hillen U, Geier J (2007a): Is incident sensitization to p-phenylenediamine related to particular exposure patterns? Results of a questionnaire study. Contact Dermatitis $\underline{56}, 266-270$

Uter W, Hildebrandt S, Geier J, Schnuch A, Lessmann H (2007b): Current patch test results in consecutive patients with, and chemical analysis of, disperse blue (DB) 106, DB 124, and the mix of DB 106 and 124. Contact Dermatitis 57, 230-234

Uter W, Gefeller O, Geier J, Schnuch A (2008): Changes of the patch test population (MOAHLFA index) in long-term participants of the Information Network of Departments of Dermatology, 1999-2006. Contact Dermatitis 푸, 56-57

Uter W, de Padua CM, Pfahlberg A, Nink K, Schnuch A, Lessmann H (2009): Kontaktallergien gegen topische Kortikosteroide - Ergebnisse des IVDK und epidemiologische Risikobewertung. J Dtsch Dermatol Ges 모 34-41

Uter W, Aberer W, Armario-Hita JC, Fernandez-Vozmediano JM, Ayala F, Balato A, Bauer A, Ballmer-Weber B, Beliauskiene A, Fortina AB (2012): Current patch test results with the European baseline series and extensions to it from the 'European Surveillance System on Contact Allergy’ network, 2007-2008. Contact Dermatitis 67, 9-19

Uter W, Warburton K, Weisshaar E, Simon D, Ballmer-Weber B, Mahler V, Fuchs T, Geier J, Wilkinson M (2016): Patch test results with rubber series in the European Surveillance System on Contact Allergies (ESSCA), 2013/14. Contact Dermatitis 75, 345-352

Veien NK, Rosner K, Skovgaard GL (2004): Is tea tree oil an important contact allergen? Contact Dermatitis $\underline{50}, 378-379$

Vena GA, Foti C, Angelini G (1994): Sulfite contact allergy. Contact Dermatitis $\underline{31}$, 172-175

Veron C, Hildebrand HF, Martin P (1986): Dental amalgams and allergy. J Biol Buccale 14, 83-100

Vogel TA, Heijnen RW, Coenraads PJ, Schuttelaar MA (2017): Two decades of pphenylenediamine and toluene-2,5-diamine patch testing - focus on co-sensitizations in the 
European baseline series and cross-reactions with chemically related substances. Contact Dermatitis $\underline{76}, 81-88$

von der Werth JM, Ratcliffe J, English JS (1999): Compositae mix is a more sensitive test for Compositae dermatitis than the sesquiterpene lactone mix. Contact Dermatitis $\underline{40}$, 273-276

Wahlberg J: Patch Testing. In: v. Rycroft RJG, Menne T, Frosch PJ (Hrsg.): Textbook of Contact Dermatitis; Springer Verlag, Berlin Heidelberg New York 1995, 239-268

Wahlberg J, Lindberg M: Patch Testing. In: Frosch PJ, Menné T, Lepoittevin J-P (Hrsg.): Contact Dermatitis. Springer Verlag, Berlin Heidelberg New York 2006, 365-390

Wakelin SH, White IR (1999): Natural rubber latex allergy. Clin Exp Dermatol 24, 245-248

Wallhäuser KH: Praxis der Sterilisation, Desinfektion, Konservierung. Georg Thieme Verlag, Stuttgart 2004

Warshaw EM, Botto NC, Maibach HI, Fowler JF Jr., Rietschel RL, Zug KA, Belsito DV, Taylor JS, DeLeo VA, Pratt MD (2009): Positive patch-test reactions to propylene glycol: a retrospective cross-sectional analysis from the North American Contact Dermatitis Group, 1996 to 2006. Dermatitis 20, 14-20

Warshaw EM, Buchholz HJ, Belsito DV, Maibach HI, Fowler JF Jr., Rietschel RL, Zug KA, Mathias CG, Pratt MD, Sasseville D (2009): Allergic patch test reactions associated with cosmetics: retrospective analysis of cross-sectional data from the North American Contact Dermatitis Group, 2001-2004. J Am Acad Dermatol 60, 23-38

Werman BS, Rietschel RL (1981): Chronic urticaria from tantalum staples. Arch Dermatol $\underline{117}, 438-439$

Wetter DA, Davis MD, Yiannias JA, Cheng JF, Connolly SM, el-Azhary RA, Farmer SA, Fett DD, Johnson JS, Linehan DL (2005): Patch test results from the Mayo Clinic Contact Dermatitis Group, 1998-2000. J Am Acad Dermatol 53, 416-421

Widmer AE, Frei R (2003): Antimicrobial activity of glucoprotamin: a clinical study of a new disinfectant for instruments. Infect Control Hosp Epidemiol 24, 762-764

Wilkinson SM, Beck MH (1996): Allergic contact dermatitis from latex rubber. $\mathrm{Br}$ J Dermatol 134, 910-914

Winhoven SM, Rutter KJ, Beck MH (2007): Toluene-2,5-diamine may be an isolated allergy in individuals sensitized by permanent hair dye. Contact Dermatitis $\underline{57}, 193$

Winter F: Handbuch der gesamten Parfumerie und Kosmetik. 2. Auflage; Springer Verlag, Wien 1932 
Worm M, Brasch J, Geier J, Uter W, Schnuch A (2005): Epikutantestung mit der DKGStandardreihe 2001-2004. Ergebnisse des IVDK. Hautarzt 56, 1114-1124

Zina AM, Fanan E, Bundino S (2000): Allergic contact dermatitis from formaldehyde and quaternium-15 in photocopier toner. Contact Dermatitis $\underline{43}, 241-242$

Zug KA, Warshaw EM, Fowler JF Jr., Maibach HI, Belsito DL, Pratt MD, Sasseville D, Storrs FJ, Taylor JS, Mathias CG (2009): Patch-test results of the North American Contact Dermatitis Group 2005-2006. Dermatitis 20, 149-160 


\section{Danksagung}

Mein besonderer Dank gilt meinem Doktorvater Prof. Dr. med. Thomas Fuchs für die Überlassung des Themas, die intensive Betreuung und Geduld bei der Fertigstellung der Arbeit.

Herrn Prof. Dr. med. Johannes Geier möchte ich einen ganz herzlichen Dank für die hervorragende Unterstützung während der gesamten Arbeit aussprechen.

Ebenso sei allen denen mein Dank ausgesprochen, die nicht namentlich erwähnt wurden, aber zum Gelingen dieser Arbeit beigetragen haben 


\section{Lebenslauf}

Ich, Mohammad Reza Safaei Nikoie, wurde am 21.09.1978 in Teheran, Iran, als Sohn von Manoochehr Safaei Nikoie und Rafat Shojai geboren.

Nach dem Abitur am Theodor Heuss Gymnasium in Göttingen begann ich im Oktober 1997 mein Studium der Humanmedizin an der Georg-August-Universität in Göttingen, und 2004 erlangte ich nach bestandenem drittem Staatsexamen die Approbation als Arzt.

Im Krankenhaus Neu-Mariahilf begann ich 2005 als Assistenzarzt in der Allgemeinchirurgie. Anschließend wechselte ich 2006 ins St. Josefskrankenhaus in Freiburg im Breisgau in die Abteilung der Inneren Medizin. Von 2008 bis 2010 arbeitete ich als Werksarzt der Firma CIBA AG in Basel, Schweiz.

In 2011 erhielt ich schließlich meinen Facharzttitel sowohl für Innere Medizin als auch für Arbeitsmedizin.

Seit 2011 arbeite ich für die pharmazeutische Industrie für die Firmen Janssen, Amgen, Seattle Genetics im Bereich der Entwicklung und Forschung von Medikamenten. Von 2017 an bin ich bei der Firma Nordic Nanovector im Bereich der Onkologie und Hämatologie tätig. 